\title{
DISSERTATION
}

\section{A CALCIUM ALUMINATE ELECTRIDE HOLLOW CATHODE}

\author{
Submitted by \\ Lauren Paula Rand \\ Department of Mechanical Engineering
}

\author{
In partial fulfillment of the requirements \\ For the Degree of Doctor of Philosophy \\ Colorado State University \\ Fort Collins, Colorado
}

Spring 2014

Doctoral Committee:

Advisor: John Williams

Melissa Reynolds

Walajabad Sampath

Azer Yalin 
Copyright by Lauren Paula Rand 2014

All Rights Reserved 


\section{ABSTRACT}

\section{A CALCIUM ALUMINATE ELECTRIDE HOLLOW CATHODE}

The development and testing of a hollow cathode utilizing C12A7 (12CaOO $\left.\mathrm{Al}_{2} \mathrm{O}_{3}\right)$ electride as an insert are presented. Hollow cathodes are an integral part of electric propulsion thrusters on satellites and ground-based plasma sources for materials engineering. The power efficiency and durability of these components are critical, especially when used in flight applications. A low work function material internal to the cathode supplies the electrons needed to create the cathode plasma. Current state-of-theart insert materials are either susceptible to poisoning or need to be heated to temperatures that result in a shortened cathode lifetime. C12A7 electride is a ceramic in which electrons contained in sub-nanometer sized lattice cages act as a conductive medium. Due to its unique atomic structure and large size, C12A7 electride has a predicted work function much lower than traditional insert materials. A novel, one-step fabrication process was developed that produced an amorphous form of C12A7 electride that had a measured work function $0.76 \mathrm{eV}$. A single electride hollow cathode was operated on xenon for over 60 hours over a two-month period that included 20 restarts and 11 chamber vent pump-down sequences with no sign of degradation, and on iodine for over 20 hours with no apparent reactivity issues. The operations of cathodes with three different orifice sizes were compared, and their effects on the interior cathode plasma modeled in a zerodimensional phenomenological model. 


\section{ACKNOWLEDGEMENTS}

The work that has gone into this dissertation has in many ways been a team effort. As such, I would like to take this opportunity to gratefully acknowledge the assistance of some of the people who made it possible. First and foremost, my advisor, John Williams, provided a huge amount of guidance in my research and I am grateful for his input. He has been a mentor to me as a scientist, as a teacher, and as a person. His utter enthusiasm for the world around him and unbridled desire to share it with everyone he meets is contagious.

I would also like to express my gratitude to my committee members. Prof. Melissa Reynolds, Prof. Walajabad Sampath, and Prof. Azer Yalin not only have served on my committee, but also made themselves available when my research crossed into their respective specialties. I was privileged to take classes from them, and each is a gifted educator.

I want to acknowledge the members of the CEPPE Laboratory for their help and support over the past five years. Daisy Williams was always who I turned to when something absolutely had to get done, and she provided enough sugar and gardening advice to last me the rest of my life. Casey and Cody Farnell were constantly willing to offer technical assistance, especially in my brief forays into coding and data acquisition. Andrew Kustas drove 15 hours with me to deliver a project when it was finished too close to a deadline to be shipped. And, of course, my thanks to the graduate students and research scientists who have come through the CEPPE Lab during my tenure for the office conversation and ping-pong games.

I want to convey my heartfelt appreciation to my family and friends for their encouragement over the past five years. My parents and brothers especially exceeded all expectations of generosity when they asked me about my research and convinced me that they really want to hear it. To my fiancé Brian, thank you for your unwavering and unconditional support. Lastly, to my grandmother: I'm sorry I didn't go to medical school. 


\section{TABLE OF CONTENTS}

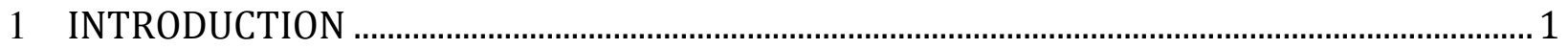

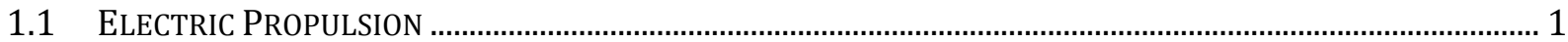

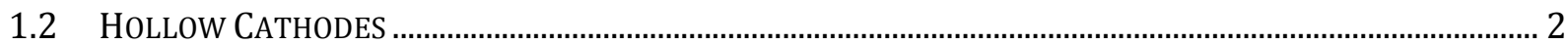

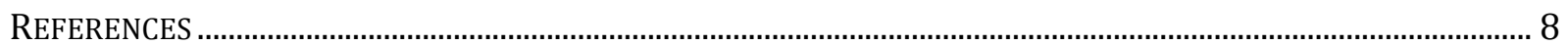

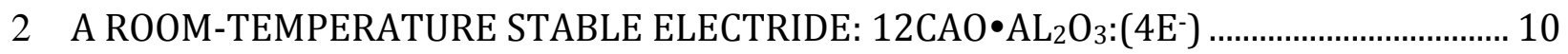

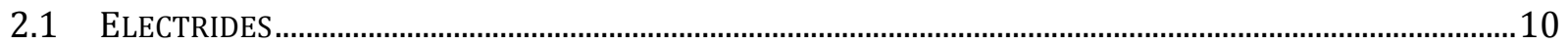

2.2 C12A7:E-

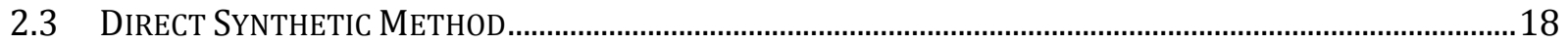

A. X-RAY DIFFRACTION CRYSTALLOGRAPHY …….................................................................................... 19

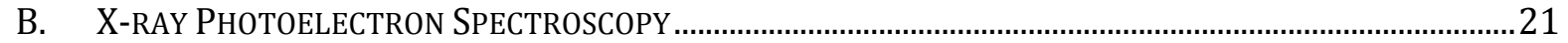

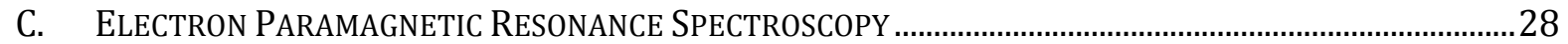

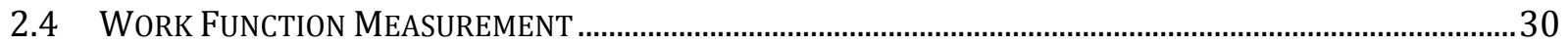

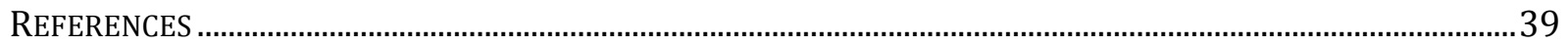

3 CATHODE FABRICATION AND APPARATUS ……………………………………………..... 41

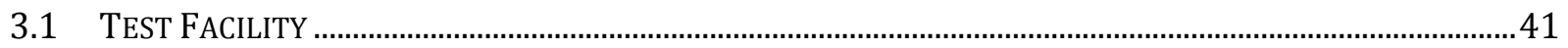

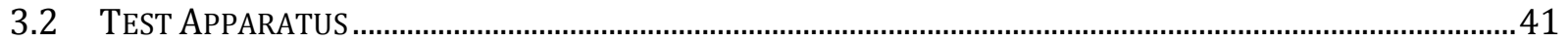

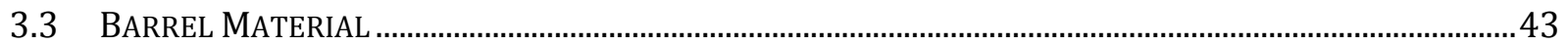

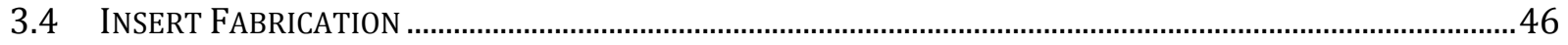

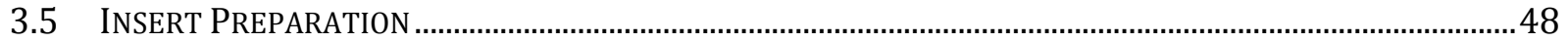

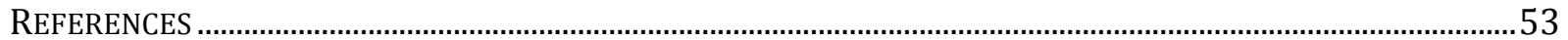

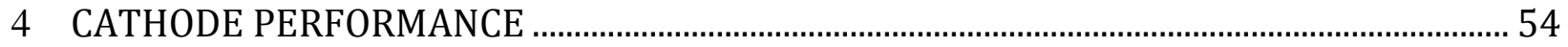

4.1 STARTING CHARACTERISTICS AND PROTOCOL................................................................................................. 54

4.2 PERFORMANCE OF THE ELECTRIDE CATHODE

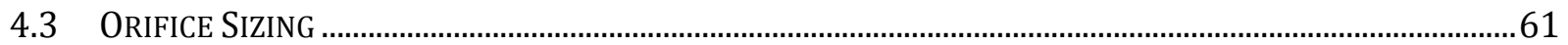

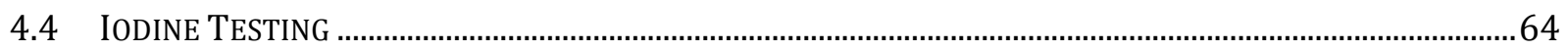

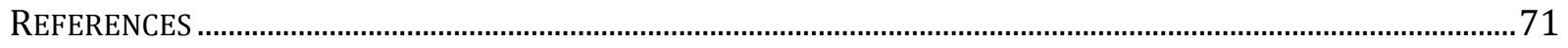

5 ZERO-DIMENSIONAL PHENOMENOLOGICAL MODEL................................................................ 72

5.1 PREvious Hollow Cathode Model ReSEARCH...........................................................................................

5.2 ZERO-DimENSIONAL ELECTRIDE HOLLOW CATHODE MODEL.......................................................................7

A. DETERMINATION OF NEUTRAL PRESSURE ………..............................................................................

B. DETERMINATION OF ELECTRON TEMPERATURE..................................................................................... 79

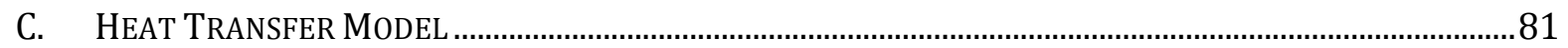

D. Calculation OF SHEath Potential ANd Plasma DENSITY …….............................................................84

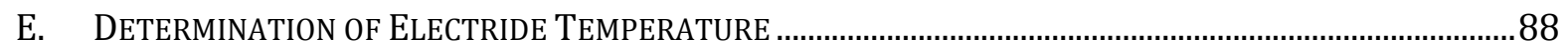

5.3 ELECTRIDE HolLOW CATHODE MOdEL RESULTS..........................................................................................94

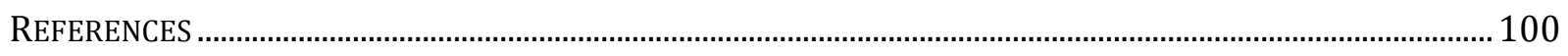

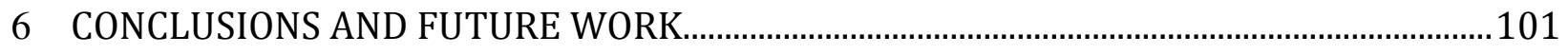

6.1 SUMMARY OF FABRICATION PROCESS ………................................................................................... 101 


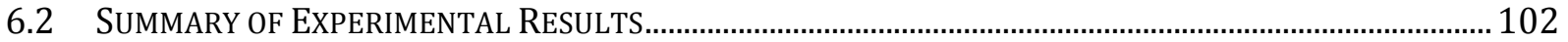

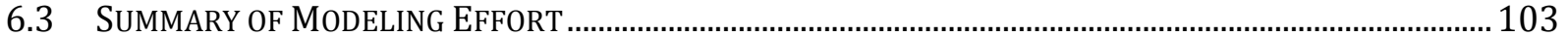

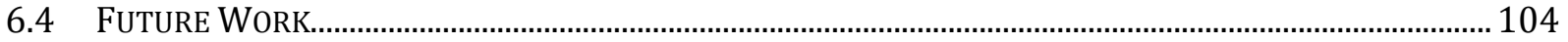

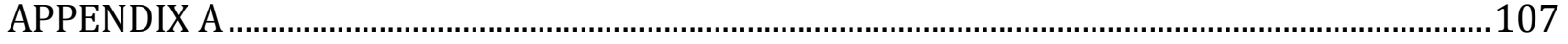

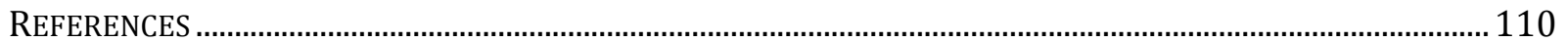




\section{LIST OF FIGURES}

FigURE 1.1: SCHEMATIC ILLUSTRATING THE OPERATION OF A HOLLOW CATHODE.................................. 4

FIGURE 2.1: BAND STRUCTURE OF METALLIC CRYSTALLINE C12A7:E

FIGURE 2.2: THE XRD SPECTRUM OBTAINED FROM A SAMPLE OF THE DIRECTLY SYNTHESIZED

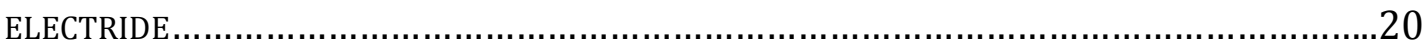

FIGURE 2.3: AN XPS SPECTRAL SWEEP OF THE DIRECTLY SYNTHESIZED ELECTRIDE SAMPLE.....................22

FIGURE 2.4: THE XPS ELEMENTAL PEAK FOR ALUMINUM IN THE DIRECTLY SYNTHESIZED ELECTRIDE

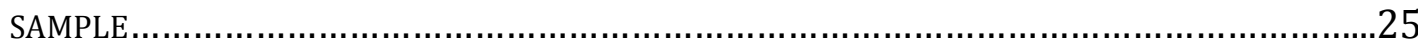

FIGURE 2.5: THE XPS ELEMENTAL PEAK FOR OXYGEN IN THE DIRECTLY SYNTHESIZED ELECTRIDE

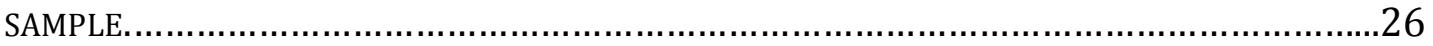

FIGURE 2.6: THE XPS ELEMENTAL PEAK FOR CALCIUM IN THE DIRECTLY SYNTHESIZED ELECTRIDE

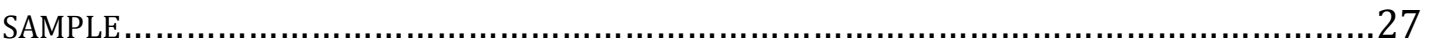

FigURE 2.7: AN UNCALIBRATED ELECTRON PARAMAGNETIC RESONANCE SPECTRUM TAKEN OF THE DIRECTLY SYNTHESIZED C12A7 ELECTRIDE SAMPLE. PLOTTED IS THE DERIVATIVE OF

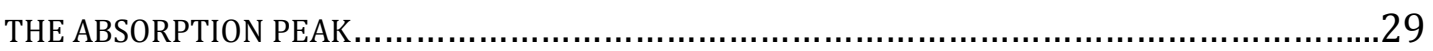

FIGURE 2.8: ROUND CRUCIBLE FABRICATED FROM EDM-3 FOR WORK FUNCTION TESTING ........................ 31

FigURE 2.9: SCHEMATIC OF THE DIODE APPARATUS USED TO MEASURE THE ELECTRIDE WORK

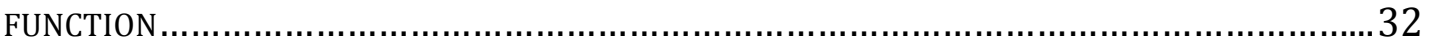

FigURE 2.10: PICTURE OF THE DIODE APPARATUS USED TO MEASURE THE ELECTRIDE WORK

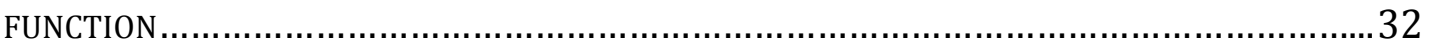

FIGURE 2.11: PEAK CURRENT DENSITIES EMITTED AT $600 \mathrm{~V}$ OVER A RANGE OF TEMPERATURES VS. TIME

FIGURE 2.12: THE CALCULATED WORK FUNCTIONS FROM THE PEAK MEASURED CURRENTS AT

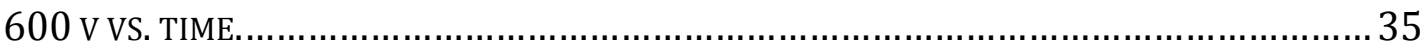

FigURE 3.1: CIRCUIT DIAGRAM OF DIODE CONFIGURATION TEST APPARATUS...................................... 42

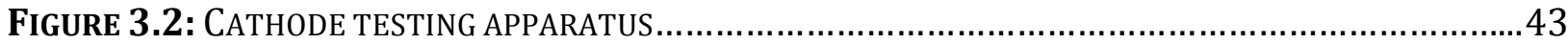

FIGURE 3.3: ELECTRIDE HOLLOW CATHODE OPERATING UNSTABLY WITH MATERIAL

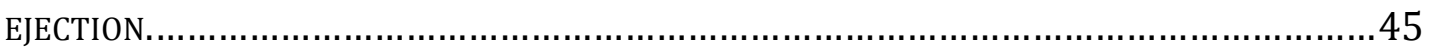

FIGURE 3.4: GRAPHITE CRUCIBLE USED TO PRODUCE ELECTRIDE ................................................... 46 
FigURE 3.5: ELECTRIDE SLIVER CUT FROM CRUCIBLE USING DIAMOND-COATED DREMEL BLADE. 46

FigURE 3.6: ANODE VOLTAGE AS A FUNCTION OF TIME FOR AN ELECTRIDE HOLLOW CATHODE WITH AN INSERT FORMED FROM A CONTINUOUS ELECTRIDE SLIVER...............................47

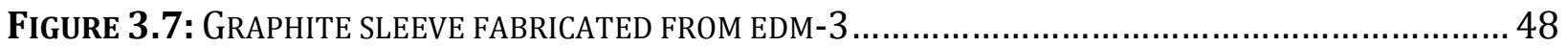

FIGURE 3.8: ANODE VOLTAGE AS A FUNCTION OF TIME OVER THE COURSE OF FOUR RUNS DURING

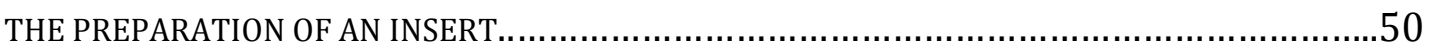

FIGURE 3.9: BARREL TEMPERATURE AS A FUNCTION OF TIME OVER THE COURSE OF FOUR RUNS DURING THE PREPARATION OF AN INSERT

FigURE 4.1: THE GEOMETRY USED TO CALCULATE THE BREAKDOWN VOLTAGE BETWEEN THE CATHODE ORIFICE PLATE AND THE KEEPER. .56

FigURE 4.2: A GRAPH OF THE ANODE VOLTAGE VERSUS TIME FOR OVER 60 HOURS OF OPERATION ON A SINGLE INSERT .59

FIGURE 4.3: A GRAPH OF THE BARREL TEMPERATURE TIME FOR OVER 60 HOURS OF OPERATION ON A SINGLE INSERT 60

FIGURE 4.4: THE MEASURED BARREL TEMPERATURE OF CATHODES WITH THREE DIFFERENT ORIFICE SIZES AS A FUNCTION OF THE DISCHARGE CURRENT 62

FIGURE 4.5: THE MEASURED BARREL TEMPERATURE OF CATHODES WITH THREE DIFFERENT ORIFICE SIZES AS A FUNCTION OF MASS FLOW RATE 62

Figure 4.6: ANODE VOLTAGE BEHAVIOR WITH THREE DIFFERENT ORIFICE SIZES AS A FUNCTION OF THE DISCHARGE CURRENT. 63

FIGURE 4.7: ANODE VOLTAGE BEHAVIOR WITH THREE DIFFERENT ORIFICE SIZES AS A FUNCTION OF MASS FLOW RATE. 63

FIGURE 4.8: AN ELECTRIDE HOLLOW CATHODE IN DIODE CONFIGURATION OPERATING ON IODINE .66

FIGURE 4.9: ANODE VOLTAGE AS A FUNCTION OF DISCHARGE CURRENT FOR AN ELECTRIDE HOLLOW CATHODE RUNNING ON IODINE.

FIGURE 4.10: ANODE VOLTAGE AS A FUNCTION OF MASS FLOW RATE FOR AN ELECTRIDE HOLLOW CATHODE RUNNING ON IODINE. 70

FigURE 5.1: SCHEMATIC OF THE ENERGY BALANCE APPLIED TO THE CONTROL VOLUME DRAWN AT THE INSERT SURFACE .82

FIGURE 5.2: A SCHEMATIC OF THE ENERGY BALANCE APPLIED TO TWO CONTROL VOLUMES .86

FIGURE 5.3: SCHEMATIC OF THE ENERGY BALANCE APPLIED TO THE CONTROL VOLUME DRAWN 
AT THE ELECTRIDE SURFACE.

FIGURE 5.4: AN ILLUSTRATION OF THE PLASMA SHEATH FORMED BETWEEN THE GRAPHITE

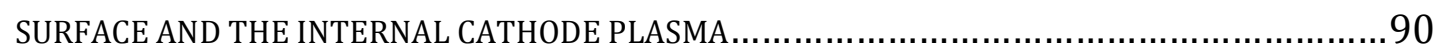

FIGURE 5.5: AN ILLUSTRATION OF THE PLASMA SHEATH FORMED AT THE INTERSECTION BETWEEN THE ELECTRIDE AND THE GRAPHITE IN AN ELECTRIDE CATHODE WITH

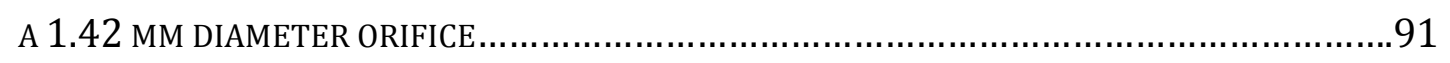

FIGURE 5.6: SCHEMATIC OF THE ENERGY BALANCE APPLIED TO THE CONTROL VOLUME DRAWN AT THE ELECTRIDE SURFACE WITH CORRECTED DIRECTIONS OF HEAT FLOW ........................93

FIGURE 5.7: THE CALCULATED INTERNAL PRESSURE AND NEUTRAL DENSITY FOR THE THREE

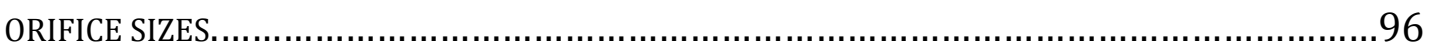

Figure 5.8: THE CALCULATED ELECTRON TEMPERATURE AND SHEATH POTENTIAL (EVALUATED OVER THE GRAPHITE) FOR THE THREE ORIFICE SIZES............................... 97

FIGURE 5.9: THE CALCULATED PLASMA DENSITY FOR THE THREE ORIFICE SIZES ..............................98

FigURE 5.10: THE CALCULATED ELECTRIDE SURFACE TEMPERATURE AND THE DIFFERENCE BETWEEN THE SURFACE TEMPERATURE AND THE MEASURED BARREL TEMPERATURE FOR THE THREE ORIFICE SIZES. 99

FigURE A. 1: H6 THRUSTER WITH MOUNTED ELECTRIDE HOLLOW CATHODE 108

FIGURE A. 2: AFRL H6 HALL THRUSTER OPERATING IN CONJUNCTION WITH ELECTRIDE HOLLOW CATHODE 109 


\section{Introduction}

This work describes efforts to fabricate and test a hollow cathode that utilizes C12A7 electride as a thermionic emission surface. Hollow cathodes are essential components of electric propulsion, and, as with any space-based technology, it is highly desirable for hollow cathodes to require minimal power to operate and be resistant to adverse conditions. In regard to these metrics, the C12A7 electride cathode developed as part of this effort compares very favorably to the current state-of-the-art. The low work function and stable structure of $\mathrm{C} 12 \mathrm{~A} 7$ electride enable a cathode to be started immediately from room temperature without a heater, which is a common point of failure in electric propulsion systems. The electride insert can be exposed to atmospheric conditions without degradation, and can be operated with reactive propellants like iodine. This is the first report, as far as the author is aware, of the use C12A7 electride in a hollow cathode. Over the course of the work described herein, approximately 10 different cathodes were fully fabricated and tested.

\subsection{Electric Propulsion}

Almost every satellite mission requires the use of in-space propulsion after launch. The ideal propulsion system is lightweight, long lasting, durable, and delivers a large impulse from a given mass of propellant. Electric propulsion utilizes electric and or magnetic fields and electric power sources to accelerate ions away from a spacecraft to produce thrust [1]. Electric propulsion was first conceived of by Tsiolkovsky 1911, who mused on the "use of electricity to produce a large velocity from the particles ejected from a rocket device" [2]. In 1917, Robert H. Goddard filed the first patent directly related to 
electric propulsion, in which he reported on the acceleration of ions through the use of an electrostatic field [3]. The first electric thruster to demonstrate operation in space was a gridded mercury ion thruster on the Space Electric Rocket Test I mission in 1964. The thruster operated for 31 minutes [4].

Although they vary in their architecture and operation, plasma-based electric propulsion thrusters accelerate ions from a plasma into a plume to produce thrust. The plasma is the result of electron-neutral collisions, in which a cathode supplies electrons that bombard neutral propellant atoms. Typically xenon propellant is used, although vaporized cesium and mercury have also been utilized [1]. The specific impulse (ISP) produced by electric propulsion devices are greater than those generated by chemical propulsion devices. More specifically, ion thrusters range in ISP from 2500 to $3600 \mathrm{~s}$, and Hall thrusters vary between 1500 and 2000 s. Chemical thrusters have ISPs closer to 150 to $450 \mathrm{~s}$. As a result, electric propulsion devices are desirable for missions that require large velocity changes. Due to limitations on the maximum power available on spacecraft, most electric propulsion systems need to be operated for long periods, and hence, electric propulsion devices must have long lifetimes [1].

\subsection{Hollow Cathodes}

The work described herein focuses on the development of a hollow cathode for electric propulsion applications. The simplest type of cathode is a hot filament, in which a conductive wire is resistively heated to a temperature at which electrons are boiled off its surface [5]. Hot filament cathodes are not suitable for flight applications due to their low durability, high power consumption, and limited lifetime. The common failure mechanism of a hot filament cathode stems from the evaporation of the cathode material at the 
elevated temperatures required for sufficient electron emission. A much more robust cathode technology is the hollow cathode.

Hollow cathodes are cylindrical in geometry and are typically constructed from a refractory metal tube with an orifice plate at the downstream end. Inside the tube is an annular insert fabricated from a low work function material. Propellant gas is fed from the upstream end of the tube that flows over the insert surface and out the orifice. The orifice serves to increase the neutral pressure inside the cathode tube, often up to several Torr depending on the orifice diameter [6]. A coaxial, sheathed heater, wrapped around the outer surface of the tube, heats the insert material to a temperature where copious electron emission can occur. A closed keeper electrode with an orifice located just downstream of the cathode orifice is biased positive and used to accelerate electrons from the cathode. The electrons collide with the neutral propellant atoms, ionize them and form plasmas within and downstream of the cathode. The desired current is drawn from these plasmas to an anode. In electric propulsion applications, the anode is either a component of the thruster, such as the back plate of a hall thruster channel, or the positively charged thruster plume [1]. The geometry and operation of a typical hollow cathode is shown in Figure 1.1.

It is desirable for the material that makes up the insert to have as low a work function as possible. The work function is the energy needed for an electron in the highest occupied electron state of a material at $0 \mathrm{~K}$ to emit sufficiently far into a vacuum that it is no longer affected by electric fields within the material. The electron must overcome the attractive forces of the surface atoms and the loss in kinetic energy due to inter-material collisions [7]. If the material is heated until the electron has enough kinetic energy to escape, the phenomenon is termed thermionic emission [8]. Thermionic emission is 


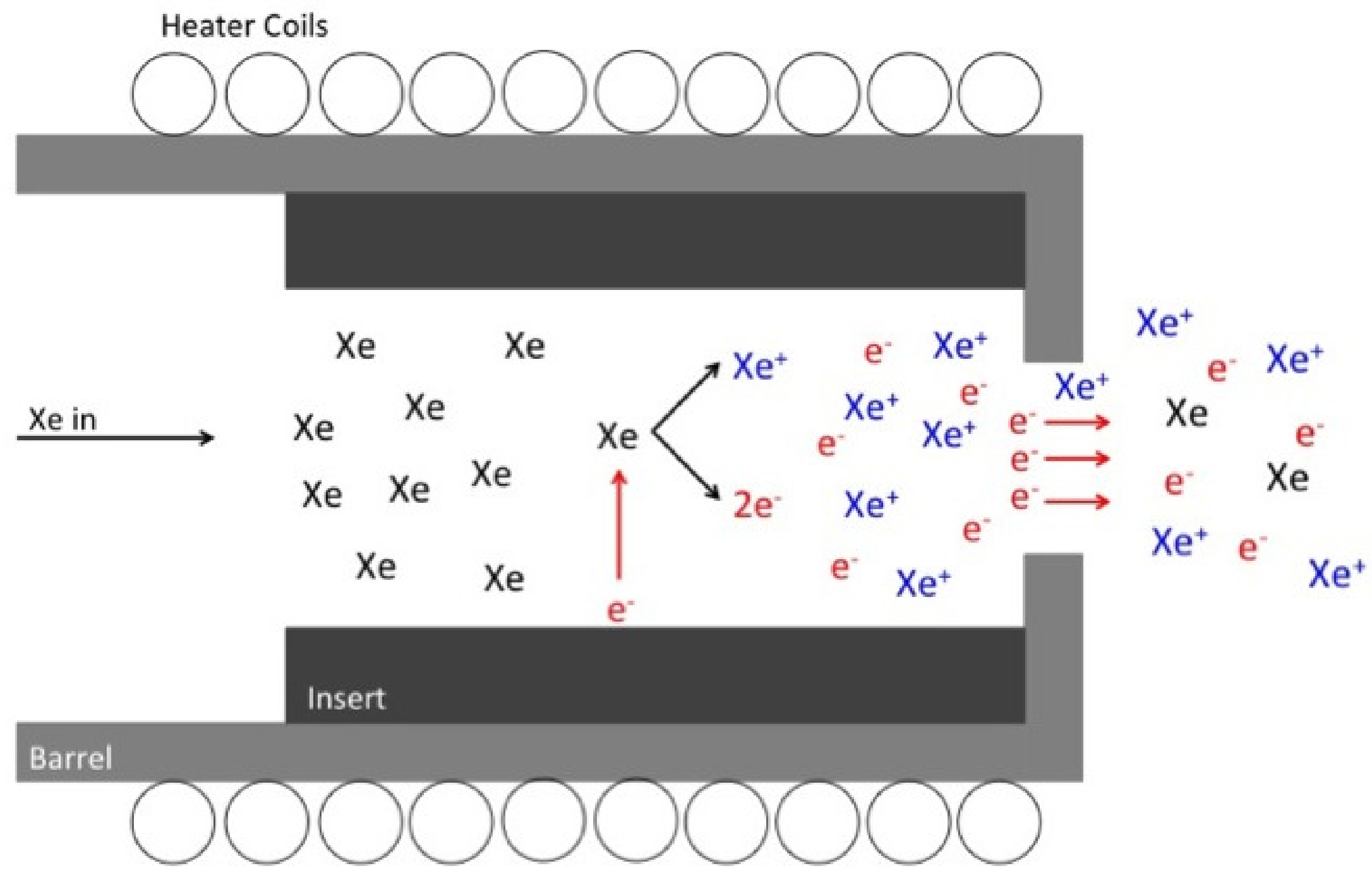

Figure 1.1: Schematic illustrating the operation of a hollow cathode. Electrons emitted from the insert ionize propellant atoms. Current is then drawn out the orifice by the anode. The keeper electrode is not shown. 
described by the Richardson-Dushman equation

$$
J=A T^{2} \exp \left(\frac{-e \phi}{k T}\right)
$$

where $\boldsymbol{J}$ is the emitted thermionic current, $\boldsymbol{A}$ is a material-specific constant, $\boldsymbol{T}$ is the material temperature, $\boldsymbol{k}$ is Boltzmann's constant, $\boldsymbol{e}$ is the charge of an electron, and $\boldsymbol{\Phi}$ is the work function [9]. It can be seen through examination of (1.1) that a material with a lower work function will emit a larger current at a given temperature than a material with a higher work function. As a result, a hollow cathode with a low work function insert will not need to be heated by the coaxial heater as hot as a cathode with a higher work function insert to emit electrons and create an internal plasma, through which the desired current can be drawn to the anode. Once a cathode has started, the bombardment of the interior surface by ions from the internal plasma self-heats the insert, allowing the heater to be turned off [1].

There are three commonly utilized insert materials in current state-of-the-art cathodes. Lanthanum hexaboride (LaB6) and cerium hexaboride (CeB6) have bulk work functions near $2.7 \mathrm{eV}$ and $2.5 \mathrm{eV}$, respectively $[10,11]$. Barium-impregnated porous tungsten $(\mathrm{BaO}-\mathrm{W})$ has a work function of $2.1 \mathrm{eV}$ [10]. Unlike LaB6 and CeB6, in which the work function is a property of the bulk material, $\mathrm{BaO}-\mathrm{W}$ inserts rely on a monolayer barium coating at the surface of the porous tungsten matrix to form a dipole with residual oxygen, lowering the potential barrier that electrons need to overcome to be emitted. Because the work function is so reliant on a specific surface state, $\mathrm{BaO}-\mathrm{W}$ inserts are susceptible to contamination by excess oxygen [12]. Additionally, the barium evaporates quickly at elevated temperatures where some cathodes operate, which limits the lifetime of the 
insert [13]. Although BaO-W inserts are sensitive to contamination and exhibit shortened lifetimes at high temperatures, they have been operated for over 50,000 hours in the NEXT lifetest [14].

To emit sufficient electrons to create an internal plasma and start the cathode, BaOW inserts must be heated to $1300 \mathrm{~K}$, and $\mathrm{LaB}_{6}$ inserts to $1900 \mathrm{~K}$ [10]. The coaxial heaters used to heat the cathodes rely on the resistive heating of a sheathed filament, and are subject to the filament breaking, the filament shorting to the sheath, and the sheath cracking [15]. The rate at which the heater fails is related to the temperature desired for the cathode to start. Thus, it is desirable to lower the temperature at which the cathode starts. If a low enough work function insert material could be found that could supply sufficient electrons to start the cathode at room temperature, the heater could be left out of the design entirely, removing a possible point of failure from the thruster.

Recently, CubeSats have gained a tremendous amount of interest within the aerospace community. CubeSats are a $1 \mathrm{~kg}$ payload within a $10 \mathrm{~cm}$ cubed box. These weight and volume restrictions allow for a "plug and play" satellite development process. Rather than spend large amounts of time and money building subsystems specific to each satellite, CubeSats are designed to be similar enough to one another that they can use "off the shelf" parts [16]. This leads to sub- $\$ 100,000$ costs to design, manufacture, and launch a CubeSat [17]. However, due to their size, CubeSats have very little power available for propulsion [18]. A hollow cathode that had no heater, could be cold-started instantly, and was inexpensive to fabricate and store would open up several possibilities for the development of a CubeSat electric propulsion thruster. 
The work described in this dissertation is discussed over the course of the following five chapters. Chapter 2 reviews the development of a novel, direct synthesis of C12A7 electride and analyzes the results of several characterization experiments. In Chapter 3, the integration of the fabricated electride into a hollow cathode is described. Chapter 4 summarizes the operation of the electride hollow cathode at a variety of gas flows, discharge currents, and on iodine. In Chapter 5, a zero-dimensional model is presented to gain insight into the internal plasma conditions of an electride hollow cathode. Chapter 6 discusses suggestions for future research. Lastly, Appendix A presents the results of a test in which a prototype version of the electride hollow cathode operated in conjunction with a $6 \mathrm{~kW}$ Hall thruster. 


\section{References}

[1] D. Goebel and I. Katz, Fundamentals of Electric Propulsion: Ion and Hall Thrusters (John Wiley \& Sons Inc, 2008).

[2] E. Choueiri, in 40th AIAA/ASME/SAE/ASEE Jt. Propuls. Conf. Exhib. (American Institute of Aeronautics and Astronautics, Reston, Virigina, 2004).

[3] R. Goddard, U.S. Patent No. US1363037 (1920).

[4] J. Sovey, V. Rawlin, and M. Patterson, J. Propuls. Power 17, (2001).

[5] J. C. Whitaker, The Electronics Handbook, 2nd ed. (CRC Press, Boca Raton, FL, 2005).

[6] I. G. Mikellides, I. Katz, D. M. Goebel, J. E. Polk, and K. K. Jameson, Phys. Plasmas 13, 063504 (2006).

[7] R. Eisberg and R. Resnick, Quantum Physics of Atoms, Molecules, Solids, Nuclei, and Particles, Second (John Wiley \& Sons Inc, New York, NY, 1985).

[8] K. L. Jensen, Electron Emission Physics, Advances i (Elsevier Inc., San Diego, California, 2007).

[9] O. W. Richardson, The Emission of Electricity From Hot Bodies (Longmans, Green and Company, 1921).

[10] D. Goebel, R. Watkins, and K. Jameson, J. Propuls. Power 23, 552 (2007).

[11] D. J. Warner, R. D. Branam, and W. A. Hargus, J. Propuls. Power 26, 130 (2010).

[12] J. Cronin, IEE Proc. I (Solid-State Electron Devices) 128, (1981).

[13] S. Kovaleski, in 27th Int. Electr. Propuls. Conf. (Pasadena, CA, 2001).

[14] R. Shastry, D. A. Herman, G. C. Soulas, and M. J. Patterson, in 33rd Int. Electr. Propuls. Conf. (Washington, DC, 2013).

[15] W. Tighe, in Vac. Electron. Conf. (Monterey, CA, 2006).

[16] K. Woellert and P. Ehrenfreund, Adv. Sp. ... 47, 663 (2011).

[17] J. Puig-Suari, C. Turner, and R. Twiggs, in 15th Annu. AIAA/USU Conf. Small Satell. (Logan, Utah, 2001). 
[18] J. Mueller, R. Hofer, and J. Ziemer, in Jt. Army-Navy-NASA-Air Force Conf. (Colorado Springs, Colorado, 2010). 


\section{A Room-Temperature Stable Electride: $12 \mathrm{CaO} \mathrm{Al}_{2} \mathrm{O}_{3}:\left(4 \mathrm{e}^{-}\right)$}

This chapter discusses electrides and focuses specifically on work done previously with C12A7 electride. Fabrication processes are examined and compared with the procedure developed as part of the work described herein. The results of analytical material studies are compared to what is found in the literature. Additionally, work function measurements are presented.

\section{$2.1 \quad$ Electrides}

It has been long known that under certain conditions, electrons may act as anions in compounds. One of the simplest examples of this phenomenon is that when dissolved in ammonia, an alkali metal such as potassium disassociates into its ionic configuration $\left(\mathrm{K}^{+}\right)$, freeing its electron [1]. An electride is the crystalline analog of this; an electron acting as an anion in an ionically-bonded compound [2]. The electron is not bound to any specific atom or molecule in the compound, and generally has a wave function loosely centered around a crystalline defect formed by an anion vacancy, known as an F-center [2]. The electrons in electrides differ from those in metals in that they are usually not completely de-localized, and cannot be thought of as a "sea of electrons" [3]. Electrides were first reported in 1990 by James L. Dye, who published the synthesis and characterization of two electrides, $\mathrm{Cs}^{+}$ (18-crown-6) ${ }_{2} \mathrm{e}^{-}$and $\mathrm{K}^{+}$(crytand[2.2.2]) $\mathrm{e}^{-}$[4]. The $e^{-}$notation following the chemical names of the two compounds indicates that electrons act as anions.

Dye's electrides were organic by necessity. For his synthesis, he needed a solvent that would solvate the cation (in this case, $\mathrm{Cs}^{+}$or $\mathrm{K}^{+}$) while staying resistant to reduction. Two solvents that met those criteria were crown ethers and cryptands [5,6]. These 
solvents were non-reducible only below $-40{ }^{\circ} \mathrm{C}$ (above which thermal decomposition led to reducible products, such as oxygen), which limited the stability of Dye's electrides to below that temperature. Additionally, Dye's electrides could not be exposed to atmosphere because air and water introduced reducible compounds (such as oxygen) that reacted with the anionic electrons [4].

\subsection{C12A7:e-}

In 2003, Matsuishi et al reported on the fabrication of the first inorganic electride stable at room-temperature in air from a $12 \mathrm{CaO} \bullet 7 \mathrm{Al}_{2} \mathrm{O}_{3}: 2 \mathrm{O}^{2-}$ crystal [7]. The calcium aluminate compound, $12 \mathrm{CaO} \bullet \mathrm{Al}_{2} \mathrm{O}_{3}$ (referred to as $\mathrm{C} 12 \mathrm{~A}$, henceforth), is a crystalline ceramic with a lattice structure that contains $12,0.4 \mathrm{~nm}$ wide nanocages per unit cell. When formed in a non-reducing environment, the nanocages clathrate, or contain, unbounded oxygen anions $\left(\mathrm{O}^{2-}\right)$. The lattice structure of each unit cell has a net +4 positive charge, so there are two $\mathrm{O}^{2-}$ anions in each cell for overall neutrality. The oxygen anions are loosely attracted to the six calcium atoms that participate in the cage structure, which distorts the cage shape slightly. In the electride form of C12A7, the oxygen atoms are removed (through various methods, discussed below) and are replaced with electrons. To maintain the overall neutrality of the compound, four electrons per unit cell are necessary, and they occupy four of the 12 cages.

The band structure of the electride features a sub-band located within the band gap between the valence band and the conduction band called the cage conduction band [810]. The cage conduction band is made up of electronic states that represent the electrons clathrated within the cages. The band structure is shown in Figure 2.1. The shaded area 
represents occupied states at $0 \mathrm{~K}$. As such, the top edge of the shaded area denotes the Fermi level [11].

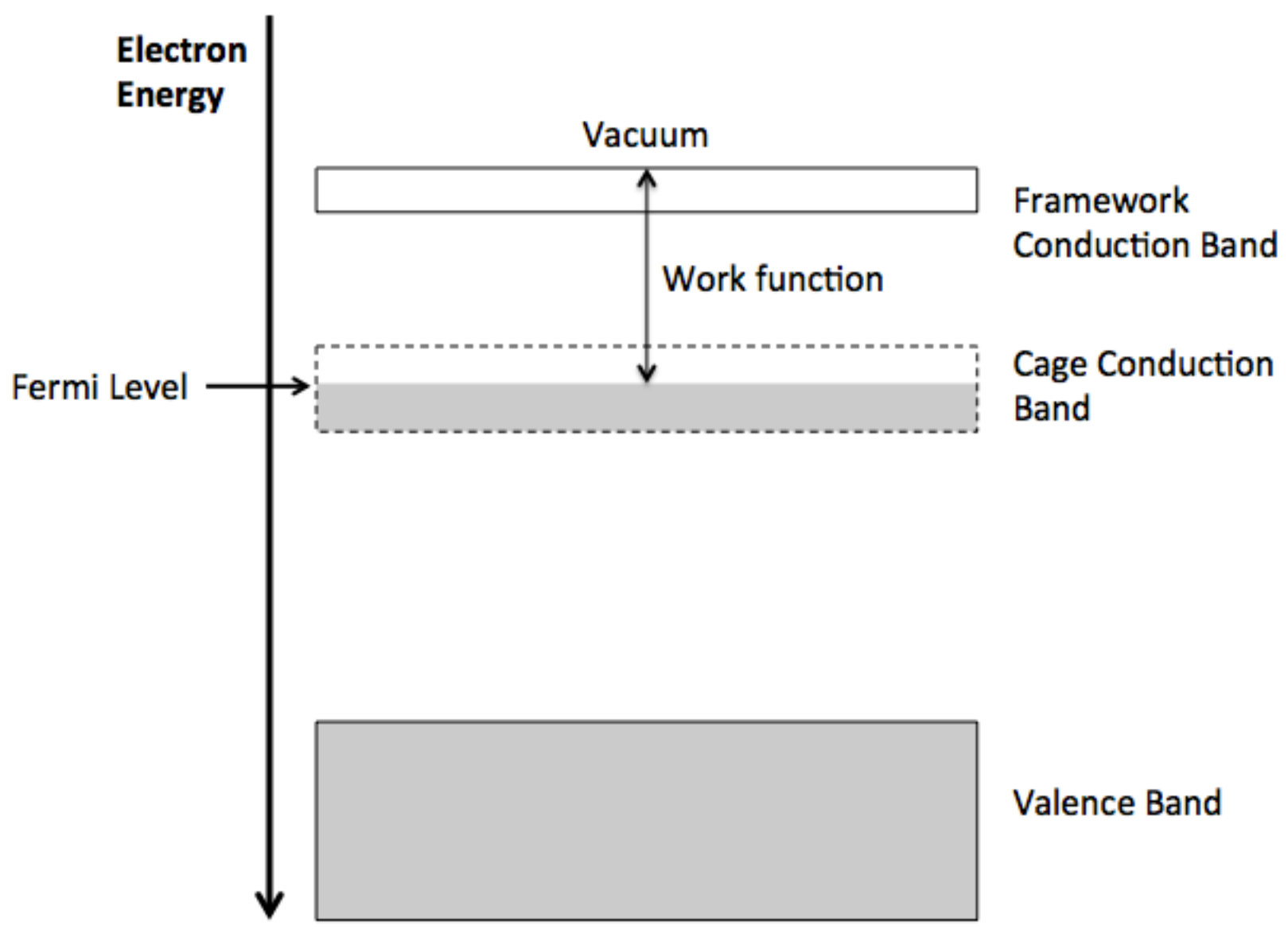

Figure 2.1: Band structure of metallic crystalline C12A7:e'.

At low concentrations, on the order of $1 \times 10^{19} \mathrm{~cm}^{-3}$ and less, conduction of the electrons through the crystal occurs via a hopping mechanism in which clathrated electrons jump between unoccupied cages [10]. The cages contract tightly around the highly-localized electrons, impeding conductivity. If an occupied cage is modeled as a particle in a potential well [3], the wave function of the electron only barely extends beyond the cage boundary, decreasing the likelihood of a tunneling event that can lead to a jump between cages. As the electron density increases, the cages relax and the wave functions extend farther beyond their boundaries. At the metallic transition that occurs at 
an electron density of $1 \times 10^{21} \mathrm{~cm}^{-3}$ [9], the cages relax to the point that there is no distortion of the walls due to occupation by electrons, and the electron wave functions extend far past the cage boundaries, leading to a greater degree of electron delocalization. This results in a much higher likelihood of a cage-hopping event, which increases the conductivity of the material.

Matsuishi et al confirmed the electride form of C12A7 with electron paramagnetic resonance spectroscopy (EPR) [7]. The EPR technique measures the absorptivity of a sample when exposed to a magnetic field. The magnetic field removes the degeneracy of the electron spin states. If there are unpaired electrons in the sample, a transition (and thus absorption peak) may occur between an aligned spin state and an anti-aligned state [12]. The oxide form of $\mathrm{C} 12 \mathrm{~A} 7$ (C12A7:02-) had a single peak identified as the two unpaired valence electrons on the clathrated oxygen (the transition is allowed because the two valence electrons are in different orbitals). The electride form C12A7:e- showed a very large peak in a different location, which was indicative of unpaired electrons in nanocages [7]. Additionally, the spectrum for the electride had only one peak, which was evidence of either an unbound electron or an electron bound to only one species of atom [12]. The location of the peak maximum, also known as the g-factor, was reported to be 1.994. The g-factor is a measure of how tightly contained the electron is. For a free electron, the g-factor is 2.002319 [13]. The similarity between the two numbers is indicative of a free-floating, unbound electron in the electride material. The localization of the electride can be quantified by the ratio of the height of the positive signal to the height of the negative signal of the derivative of the absorption peak. Matsuishi et al reported a 
ratio of 2.6. Any ratio over unity is indicative of non-localized electrons, which suggests that the electride sample showed characteristics of a metallic conductor [14].

There are several methods to fabricate $\mathrm{C} 12 \mathrm{~A} 7$ electride reported in the literature. One process converted an initial synthesis of C12A7:02- to the electride form [7]. The challenging step in the synthesis process was the replacement of the oxygen anions with electrons. Matsuishi et al exposed a C12A7:02- crystal to calcium pellets heated to a temperature of $700^{\circ} \mathrm{C}$ to form hot calcium vapor, which pulled the oxygen anions out of the cages to form calcium oxide. The conductivity of the resulting electride increased with the amount of time it was exposed to the hot calcium, until 240 hours at which point it reached a maximum electron density (evidenced by a maximum conductivity) of $2 \times 10^{21} \mathrm{~cm}^{-3}$ [7]. Matsuishi et al hypothesized that this saturation limit was representative of the point at which all the oxygen anions were replaced by electrons, which was theoretically calculated to occur at an electron density of $2.33 \times 10^{21} \mathrm{~cm}^{-3}$, very close to the experimentally measured value. The downsides of this method were that it could not be done on a largescale and an insulating calcium oxide layer was left on the surface that had to be removed afterward [15].

Two additional synthetic methods more easily adapted for to large scale fabrication were reported in 2005 [16]. They both involved the use of what Hosono et al [8] called the reduced melt, which was the result of the precursors, $\mathrm{CaO}$ and $\mathrm{Al}_{2} \mathrm{O}_{3}$, being heated to 1600 ${ }^{\circ} \mathrm{C}$ in a semi-airtight carbon crucible $[17,18]$. In the melt-solidification procedure, the reduced melt was cooled slowly to room temperature, resulting in the formation of the non-conductive eutectic phase of calcium aluminate $\left(3 \mathrm{CaO} \bullet \mathrm{Al}_{2} \mathrm{O}_{3}+\mathrm{CaO} \bullet \mathrm{Al}_{2} \mathrm{O}_{3}\right)$. When the compound was heated once again to $1600{ }^{\circ} \mathrm{C}$ in the carbon crucible and cooled slowly to 
room temperature, the resulting product was crystalline electride [8]. In the glass-ceramic procedure, the reduced melt was quickly quenched in air to room temperature. The resulting product was a clear, insulative, amorphous glass. When the glass was brought above the crystallization temperature $\left(1000^{\circ} \mathrm{C}\right)$ in vacuum and slowly cooled, the resulting product was electride [8].

In both procedures, it was found that the carbon crucible was necessary for the successful formation of electride. Raman spectroscopy of the intermediates produced in the two processes, the eutectic and the reduced glass, showed a peak identified to be $\mathrm{C}_{2}{ }^{2-}$ [18]. Kim et al believed that the carbon anions were dissolved in the reduced melt following the initial heating process to neutralize the compound once the oxygen anions were removed (the hot carbon crucible formed a strongly reducing atmosphere). Because the carbon peak disappeared in the raman spectra taken after the compound was crystallized (after the second phases of the syntheses) [18], Kim et al hypothesized that carbon was not a stable clathrated species, hence the resulting formation of electride.

In 2011, Kim et al reported on a conductive, amorphous form of C12A7 electride [19]. When the reduced melt was quenched in an oxygen-deficient atmosphere, an amorphous, conductive glass formed. X-ray diffraction spectra taken of the resulting product had few sharp peaks, confirming the low crystallinity content [19]. Kim et al concluded that this electride form consisted of interconnected nanocages suspended in an amorphous matrix of $\mathrm{AlO}_{4}$ groups [19]. Because of the decreased number of cages (as compared to the fully crystalline form), the amorphous electride exhibited a conduction mechanism more akin to the semi-localized cage-hopping seen with the lower electron 
densities in the fully crystallized electride than the metallic behavior associated with higher electron densities $[9,10]$.

Dye pointed out early on in electride research that these materials should have a low work function due to the large lattice structure. Similar low work function predictions were made for $\mathrm{C} 12 \mathrm{~A} 7: \mathrm{e}^{-}$and several different measurement techniques were used to quantify the work function of the crystalline electride. In reference [18], Kim et al measured the current emitted through field-assisted thermionic emission. In this phenomenon, electrons are given kinetic energy through the temperature of the lattice structure, and an external electric field acts to artificially lower the Schottky potential barrier at the material surface. The emitted current can be calculated as a function of the temperature, work function, and applied electric field with the Richardson-Dushman equation

$$
J=A T^{2} \exp \left(\frac{-e \phi}{k T}\right) \exp \left(\frac{e}{k T} \sqrt{\frac{e E}{4 \pi \varepsilon_{o}}}\right)
$$

Kim et al measured the current emission as a function of extraction voltage at seven different temperatures. Using the analysis method discussed by Cronin [20], linear fits were made to the data when graphed on a $\ln (J)$ vs. $V^{1 / 2}$ set of axes. The y-intercepts of the trend lines were effectively the thermionic-limited emission with no surface electric field, which is a physically impossible value to measure directly because of space-charge limiting effects. The intercepts are then plotted on a $\ln \left(\mathrm{J} / \mathrm{T}^{2}\right)$ vs. $1 / \mathrm{T}$ graph. The slope of the line, according to the Richardson-Dushman equation for thermionic emission (equation (2.1)), is the work function. Kim et al reported a work function of $0.6 \mathrm{eV}$ measured with this technique [18]. However, with the same technique but a different sample a year later, Toda 
et al measured a work function of $2.1 \mathrm{eV}[21]$. The authors speculated that surface contaminants could have been responsible for reducing the work function in the original measurement.

Another measurement technique used to measure the work function of crystalline electride focuses on the current emitted through the photoemission mechanism. In this process, a photon interacts with a surface electron, imparting enough energy to the electron for it to overcome the surface potential barrier and emit into vacuum [22]. In reference [9], the authors measured the work function using photoelectron yield spectroscopy (PYS) and ultraviolet photoelectron spectroscopy (UPS). In UPS, the kinetic energies of electrons photoemitted from an electride sample when exposed to a fixed wavelength of ultraviolet light are measured. Only electrons emitted through secondary electron emission, which are thermalized through inter-material collisions and thus are no longer dependent on binding energies, are examined. As a result, the kinetic energies of the observed electrons are dependent only on their thermal temperature distribution and the work function. When the onset of the secondary electron emission peak (when graphed as intensity versus kinetic energy) is compared with that of a sample with known work function, the work function of the electride can be deduced [23]. In PYS, the emitted current from the electride is measured as a function of the wavelength of the incident beam [24]. When the square root of the emitted current is graphed versus the photon energy, the $\mathrm{x}$-intercept of the linear trend line fit to the data is the work function. Toda et al measured a work function of $2.4 \mathrm{eV}$ with both UPS and PYS [9]. 


\subsection{Direct Synthetic Method}

In early 2012 we developed a novel process to fabricate C12A7 electride. The method consists of only one heating cycle, no quenching, and is adaptable to large-scale production. A vacuum furnace is used, capable of heating a hot zone $2.54 \mathrm{~cm}$ in diameter and approximately $5 \mathrm{~cm}$ long to $1800{ }^{\circ} \mathrm{C}$. The furnace consists of a $0.05 \mathrm{~mm}$ thick piece of tantalum foil sheet wrapped around a $3.18 \mathrm{~cm}$ outer diameter $\mathrm{Al}_{2} \mathrm{O}_{3}$ tube. The tube and tantalum sheet were insulated with carbon felt. The precursors reported in reference [8] were mixed in the C12A7 stoichiometric ratio with a mortar and pestle. The powders used were 150 Mesh Type 507C aluminum oxide from Sigma Aldrich and 99.9\% pure calcium carbonate from Fisher Scientific. The mixture was deposited in a graphite crucible, fabricated from EDM-3 fine-grained graphite obtained from Ohio Carbon Blank, Inc. [25]. A graphite plate was secured over the top of the crucible with tantalum wire to keep the material contained within the crucible during the heating process. A high surface tension was observed in early fabrication attempts, and without the lid, the molten precursors often flowed up and out of the crucible.

The furnace and crucible were placed in a $0.76 \mathrm{~m}$ diameter, $0.6 \mathrm{~m}$ long stainless steel chamber pumped by a Brooks CTI-Cryogenics Cryo-Torr 10 cryopump. A Sorensen DCR40-250A $10 \mathrm{~kW}$ power supply was used to heat the tantalum sheet in the furnace. The furnace temperature was slowly raised to $1700{ }^{\circ} \mathrm{C}$ over the course of 2.15 hours, at which point the power supply was abruptly turned off and the furnace and crucible were allowed to radiatively cool to the water-cooled chamber walls. The crucible cooled to below the recrystallization temperature of $1000{ }^{\circ} \mathrm{C}$ in less than 30 minutes [8]. The chamber was 
generally not vented for at least 16 hours after the power supply had been shut off, in order to give the furnace and crucible time to cool before exposure to atmosphere.

The product of this fabrication process was metallic-looking, conductive, and bonded to the graphite. Chapter 3 presents details about how this material was used as an emission surface suitable for a hollow cathode. To positively identify the product, several analytical surface techniques were used, including EPR, x-ray photoelectron spectroscopy (XPS), and x-ray diffraction crystallography (XRD). Additionally, the work function of the product was measured.

\section{A. X-ray Diffraction Crystallography}

XRD is a technique useful in obtaining information about the crystal structure of a sample. Measurements of the angles and intensities of $x$-rays diffracted by the lattice structure of a compound give information regarding its crystalline characteristics [26]. A directly synthesized electride sample was studied in the Central Instrumentation Facility at Colorado State University with a Rigaku SAXS instrument. The spectrum is shown in Figure 2.2. The only discernable peaks were attributed to graphite, which was a likely contaminant. Other than the carbon, there appeared to be little discernable crystal structure, indicating that if the sample was indeed electride, it was amorphous. No attempt was made to recrystallize the amorphous material, which is an area that warrants further investigation. 


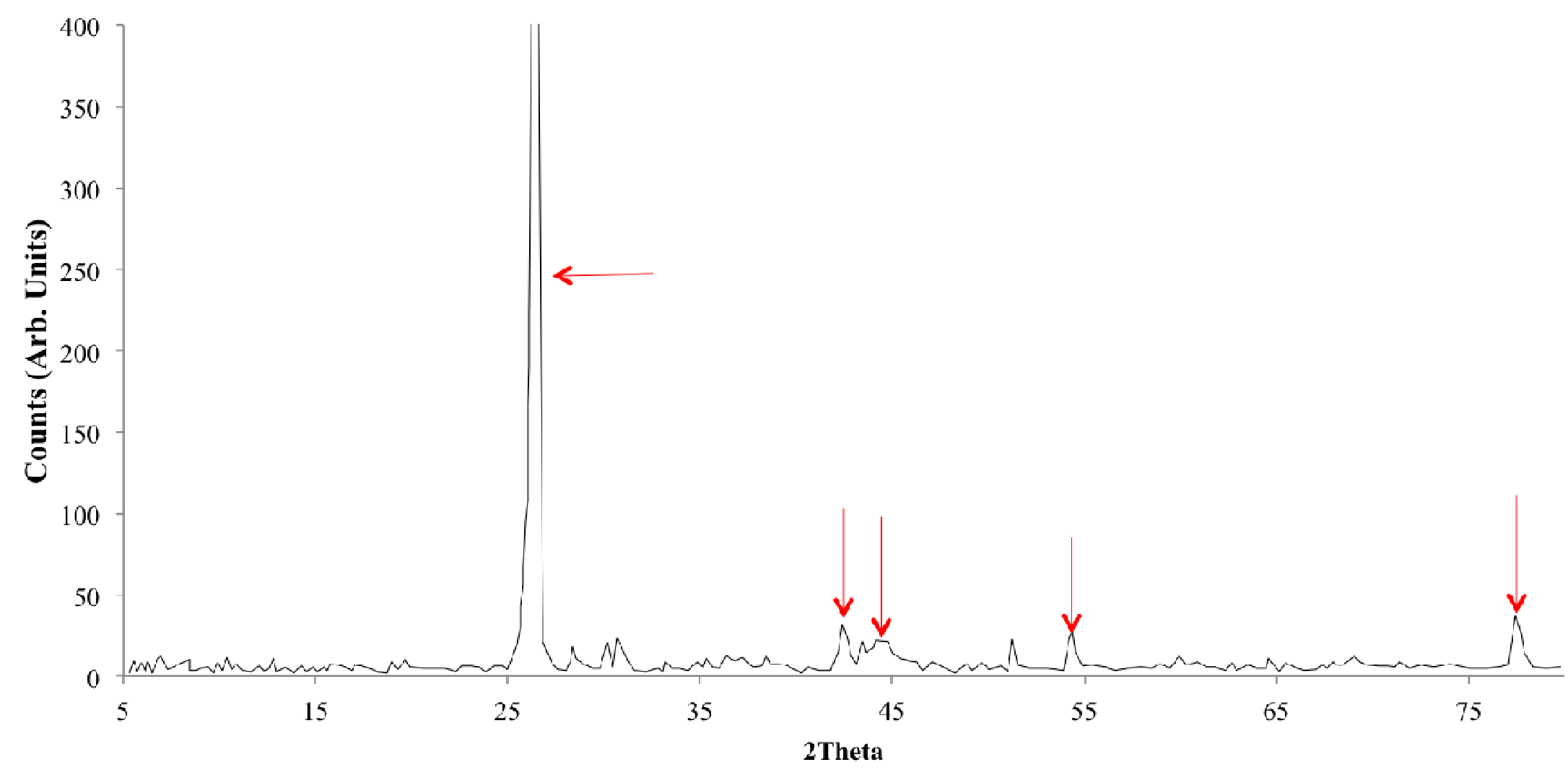

Figure 2.2: The XRD spectrum obtained from a sample of the directly synthesized electride. The red arrows indicate peaks attributed to graphite. The graphite peak at $26^{\circ}$ peaks above the cutoff of the graph at 735 counts. There are otherwise no identifiable peaks. 


\section{B. X-ray Photoelectron Spectroscopy}

XPS can be used to acquire the atomic composition of a material surface as well as binding information about the identified species. XPS is similar to UPS in that it measures the kinetic energy of electrons emitted when the sample is exposed to an incident photon beam. From that measurement, the binding energies of the electrons prior to the absorption of the incident photon can be deduced. As a result, it is possible to obtain the identity of an unknown compound [27]. An electride sample produced using the direct synthesis technique was studied with a PE-5800 x-ray photoelectron spectrometer. In an effort to remove contaminants, the sample surface was sputter cleaned with a $2 \mathrm{keV}$ argon beam to remove approximately the top micron, which was thought to be a sufficient amount of sputtering to reach a steady-state surface composition. The spectral sweep XPS spectrum obtained after the sputter cleaning is shown in Figure 2.3. Table 2.1 shows the acquired atomic composition.

Table 2.1: Atomic composition acquired from XPS spectral sweep

\begin{tabular}{|c|c|}
\hline \multicolumn{2}{|c|}{ Atomic \% } \\
\hline $\mathrm{C} 1 \mathrm{~s}$ & 85.1 \\
\hline $\mathrm{O} 1 \mathrm{~s}$ & 9.9 \\
\hline $\mathrm{Al} 2 \mathrm{p}$ & 2.9 \\
\hline $\mathrm{Ca} 2 \mathrm{p}$ & 1.5 \\
\hline
\end{tabular}




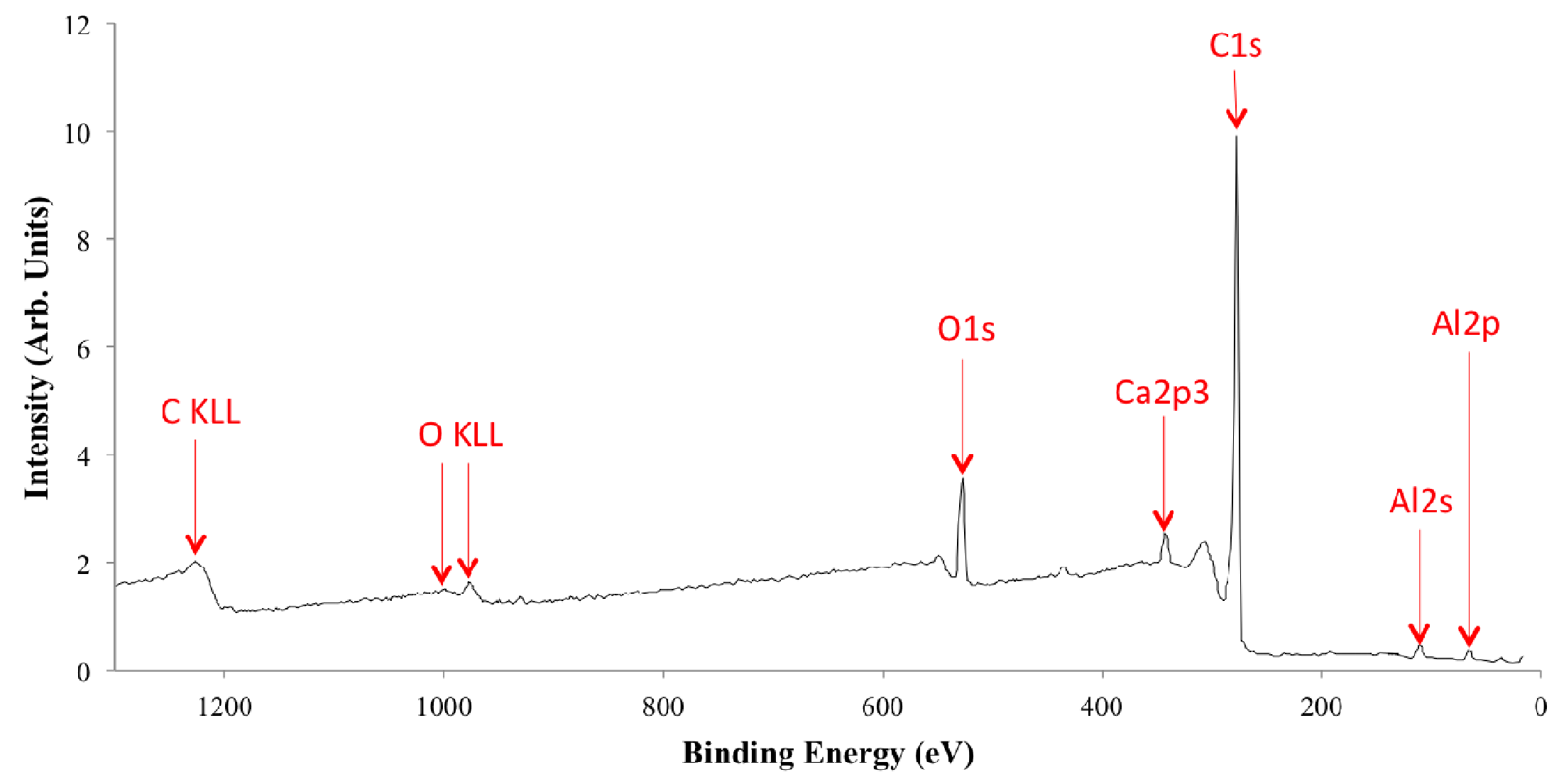

Figure 2.3: An XPS spectral sweep of the directly synthesized electride sample. 
The atomic composition indicates that $85 \%$ of the surface is carbon, which is unsurprising given that the sample was formed in a graphite crucible and separated from that crucible with a dremel blade, which created an graphite debris. The ratio of aluminum to calcium is 1.93 , which at first glance appears to rule out $12 \mathrm{CaO} \cdot 7 \mathrm{Al}_{2} \mathrm{O}_{3}$, which has an aluminum to calcium ratio of 1.17. However, the initial sputter cleaning process is an important consideration because preferential sputtering will alter the surface composition of the sample. Using the SRIM software by Ziegler, it was shown that one would expect an aluminum to calcium ratio of 2.3 , which is only $20 \%$ higher than the measured ratio.

Information regarding the chemical bonding structure of the sample can be obtained by examining the fine features of the elemental peaks. The elemental peak for aluminum is shown in Figure 2.4. The identified peaks occurred at binding energies of $74.46 \mathrm{eV}, 75.99$ $\mathrm{eV}$, and $77.55 \mathrm{eV}$. If the range of possible compounds is limited to one consisting of only the elements detected by the full spectral sweep, only $\mathrm{Al}_{2} \mathrm{O}_{3}$ and $\mathrm{Al}(\mathrm{OH})_{3}$ match the peak signature to within $\pm 0.1 \mathrm{eV}[28]$.

The elemental peak for oxygen is shown in Figure 2.5. The identified peaks occurred at binding energies of $531.31 \mathrm{eV}, 532.96 \mathrm{eV}$, and $534.72 \mathrm{eV}$. Both $\mathrm{Al}_{2} \mathrm{O}_{3}$ and $\mathrm{Al}(\mathrm{OH})_{3}$ match this peak signature to within $\pm 0.1 \mathrm{eV}$ [28].

The elemental peak for calcium is shown in Figure 2.6. The identified peaks occurred at binding energies of $348.11 \mathrm{eV}, 349.83 \mathrm{eV}, 351.74 \mathrm{eV}$, and $353.36 \mathrm{eV}$. If only the species detected in the full spectrum sweep are considered, only $\mathrm{CaO}$ and $\mathrm{CaCO}_{3}$ match the peak signature to within $\pm 0.1 \mathrm{eV}[28]$.

The identified peaks in the elemental carbon spectrum (not shown) occurred at binding energies of $283.71 \mathrm{eV}, 284.80 \mathrm{eV}, 285.67 \mathrm{eV}$, and $288.29 \mathrm{eV}$. Because 
contamination is the most likely source of the carbon, attempting to find a single compound that fits that peak profile is not useful. Instead, the carbon peak profile can be used to rule out the possibility of $\mathrm{CaCO}_{3}$ raised by the analysis of the calcium elemental peak. To within $\pm 0.1 \mathrm{eV}$, none of the carbon peaks measured in the profile match that of $\mathrm{CaCO}_{3}$, which confirms the presence of $\mathrm{CaO}[28]$.

The analysis of the elemental peaks from the XPS measurement confirms the presence of $\mathrm{CaO}$ and either $\mathrm{Al}_{2} \mathrm{O}_{3}$ or $\mathrm{Al}(\mathrm{OH})_{3}$. The possibility of aluminum hydroxide over alumina is highly unlikely, given the conductive nature of the sample, the signal seen on the EPR spectrum (presented in section C), and the successful operation of the electride hollow cathode. 


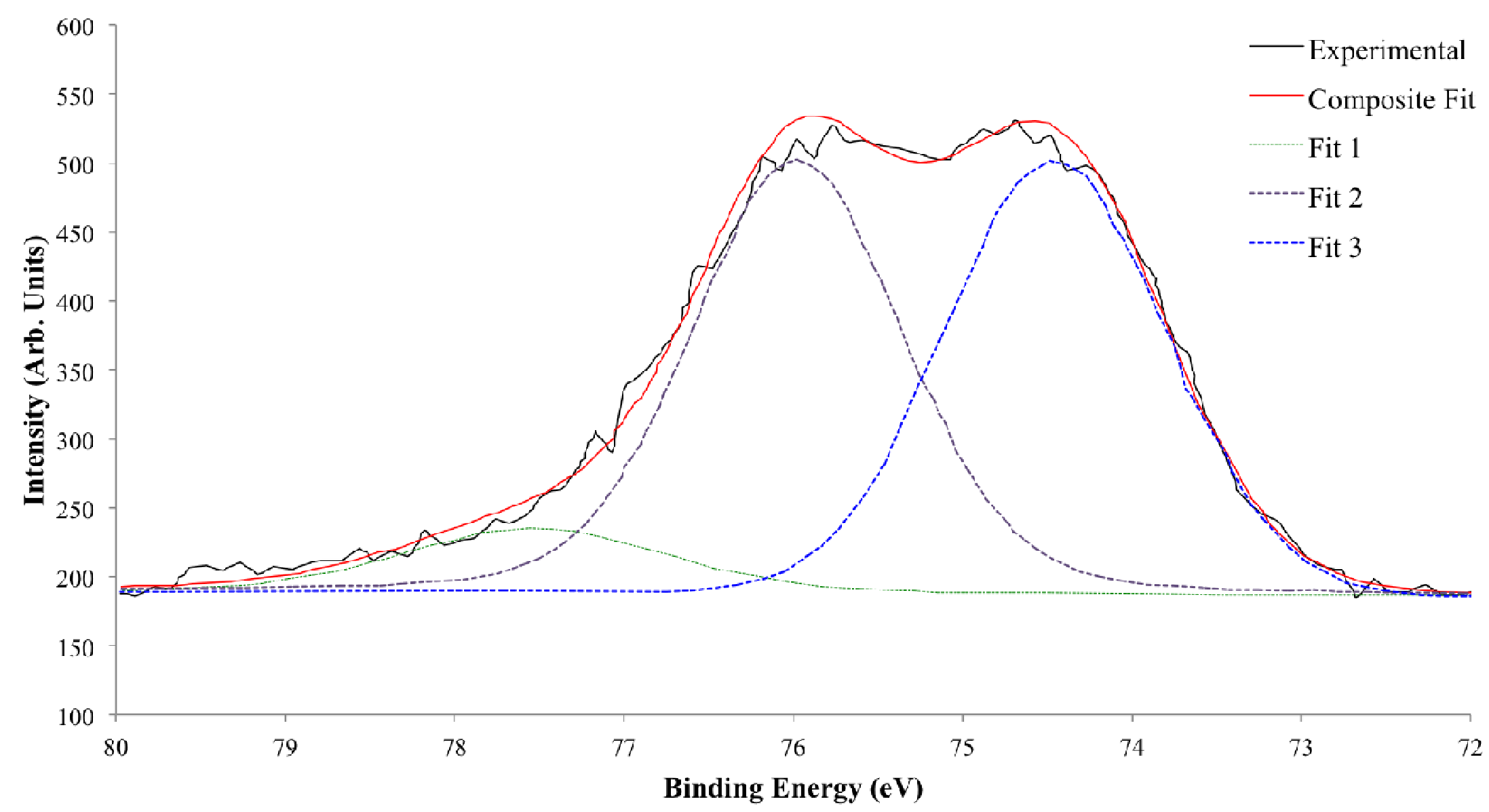

Figure 2.4: The XPS elemental peak for aluminum in the directly synthesized electride sample. The experimental data is shown in black. Three individual curve fits are superimposed over the experimental data, and their composite is shown in red. 


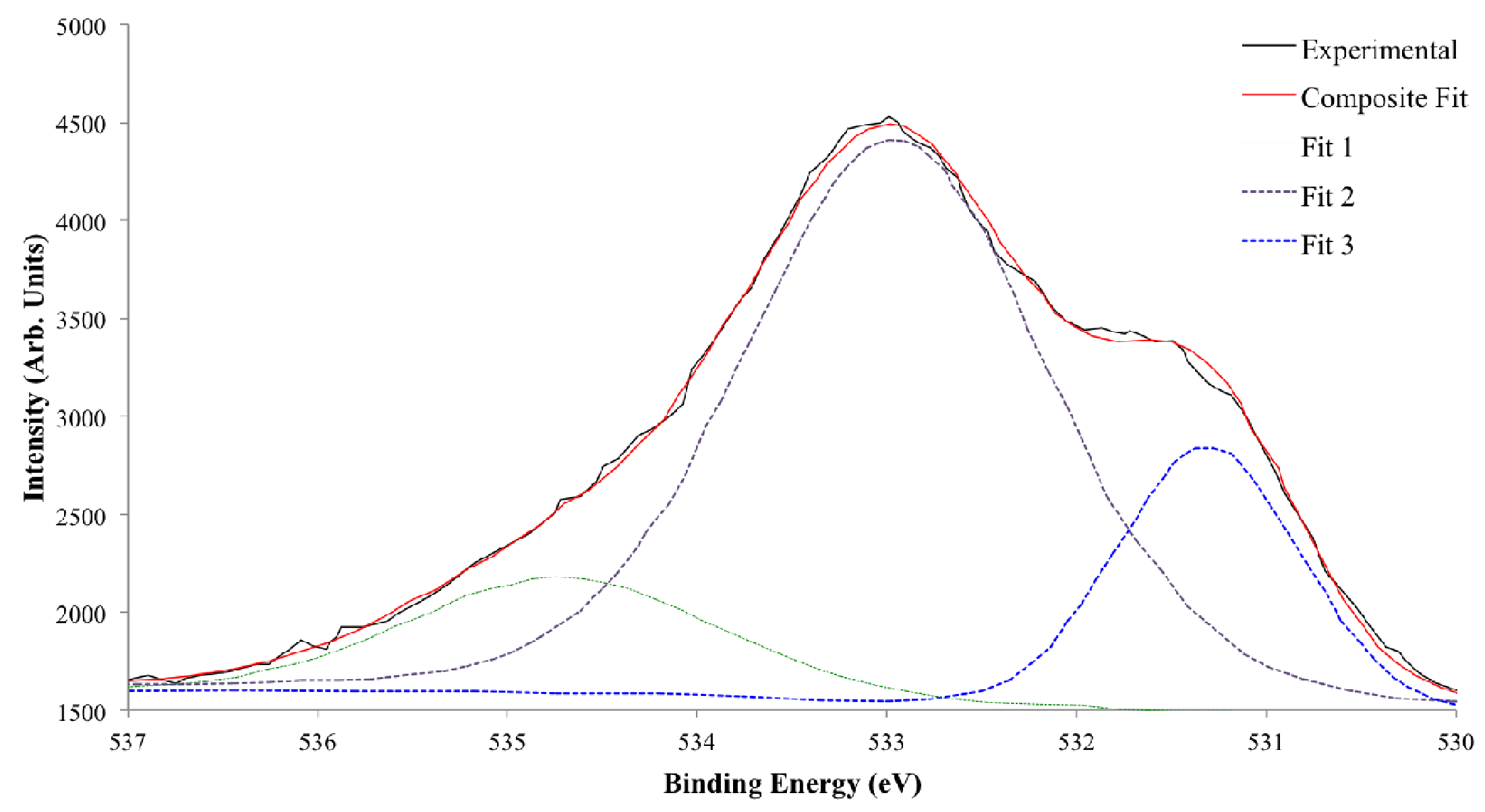

Figure 2.5: The XPS elemental peak for oxygen in the directly synthesized electride sample. The experimental data is shown in black. Three individual curve fits are superimposed over the experimental data, and their composite is shown in red. 


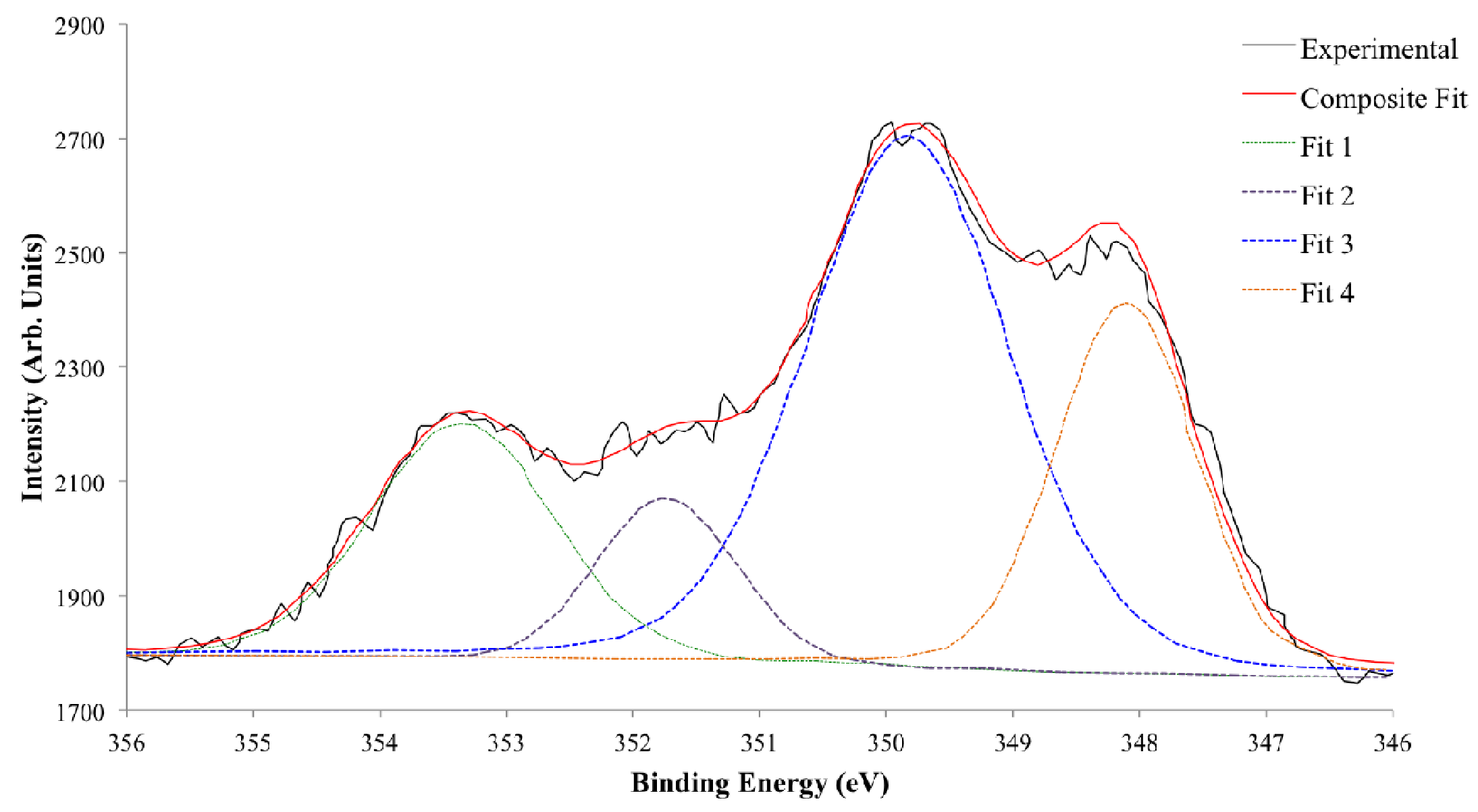

Figure 2.6: The XPS elemental peak for calcium in the directly synthesized electride sample. The experimental data is shown in black. Four individual curve fits are superimposed over the experimental data, and their composite is shown in red. 


\section{Electron Paramagnetic Resonance Spectroscopy}

As discussed previously, EPR spectroscopy can be used to detect the presence of unpaired electrons, and it also can distinguish between bound and clathrated electrons. Consequently, it yields useful information when used to examine an electride. The EPR spectrometer used is a Bruker ESR-3000. The spectrometer directs a beam of microwaves at $9.75 \mathrm{GHz}$ at the sample, and measures the absorption over a range of applied magnetic fields from $0.328 \mathrm{~T}$ to $0.368 \mathrm{~T}$. When exposed to a magnetic field, opposing electron spin states become non-degenerate due to the Zeeman effect [12]. Because of the induced energy difference, a transition may occur when an electron with a spin parallel to the magnetic field absorbs a photon and jumps to an antiparallel spin state, according to equation (2.2)

$$
h v=g \mu_{B} B_{o}
$$

where $\boldsymbol{v}$ is the frequency of the microwave, $\boldsymbol{h}$ is Planck's constant, $\boldsymbol{g}$ is the g-factor previously discussed, $\boldsymbol{\mu}_{\boldsymbol{B}}$ is the Bohr magneton, and $\boldsymbol{B}_{\boldsymbol{o}}$ is the magnetic field resonant to the transition. However, this transition can only occur if the electron is unpaired. Otherwise, there is already an electron occupying the antiparallel spin state and the Pauli exclusion principle prohibits the transition. The result of equation (2.2) is that the absorption of the microwave can be directly linked to the presence of unpaired electrons in the sample. In the case of $\mathrm{C} 12 \mathrm{~A} 7$, an absorptivity peak is indicative of either the electride form or the presence of clathrated free radicals, such as $\mathrm{O}^{2-}$. The peak of the absorption signal is the $\mathrm{g}$ factor. Figure 2.7 shows the EPR spectrum taken of the directly synthesized electride. 


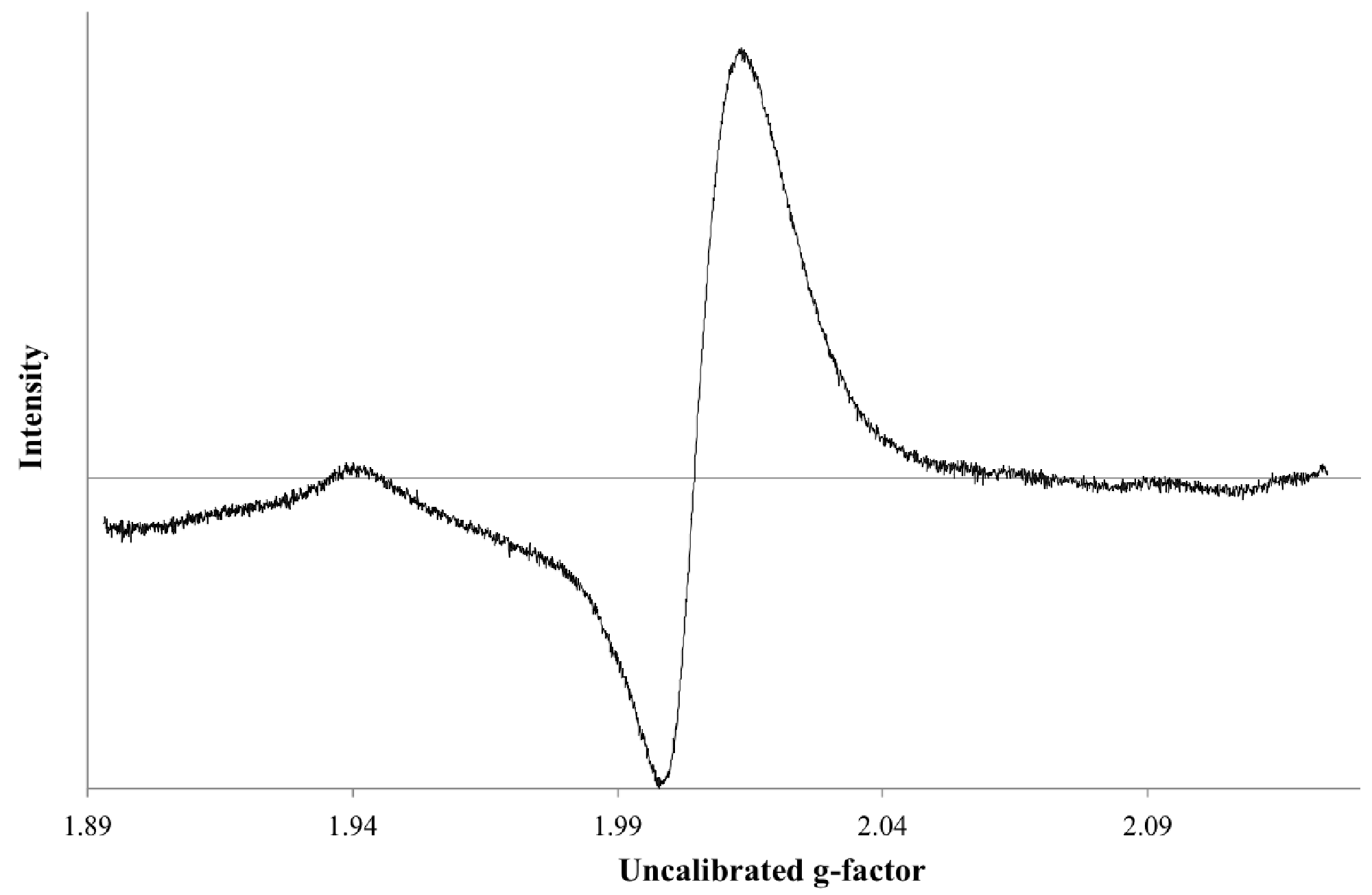

Figure 2.7: An uncalibrated electron paramagnetic resonance spectrum taken of the directly synthesized C12A7 electride sample. Plotted is the derivative of the absorption peak. When calibrated, the g-factor is 1.9376. 
There was only one peak observed in the absorption EPR spectrum, which is indicative of either an unbound electron or an electron bound to only one species of atom. The g-factor, when calibrated with a DPPH standard, was found to be 1.9376 . This is smaller than the g-factor of electride reported in the literature, and is farther away from the free electron g-factor by $2.8 \%$, indicating that the electron in the directly synthesized electride sample is more tightly contained than that reported in the literature. This is possibly due to the difference in crystallinity content; the literature sample was fully crystalline and the directly synthesized sample was amorphous, as suggested by the XRD analysis. The lack of unoccupied nanocages around the clathrated electrons may have allowed a greater contraction of the occupied cages, which would increase the discrepancy between the measured absorption peak g-factor and that of a free electron. The ratio of the positive signal height to the negative signal height is calculated to be 1.42 . This signifies some delocalization of the electrons and metallic behavior, although not to the extent reported for the fully crystalline electride $[7,12]$.

\subsection{Work Function Measurement}

As will be discussed in Chapter 3, the electride cathodes were observed to run at lower anode voltages and temperatures after several hours and multiple restarts. It was thought that there might have been a cleaning or conditioning effect caused by exposure to the internal cathode plasma that enhanced the emission properties of the electride. Hence, to obtain a relevant work function measurement, we believe it is desirable to first expose the emission surface to a cathode plasma environment. Cylindrical crucibles that could be inserted into a $6.35 \mathrm{~mm}$ diameter tantalum orificed tube were fabricated from EDM-3 fine- 
grained graphite [25]. The crucibles had a base diameter that was slightly smaller than the inner diameter of the tantalum tube. The crucible is shown in Figure 2.8.

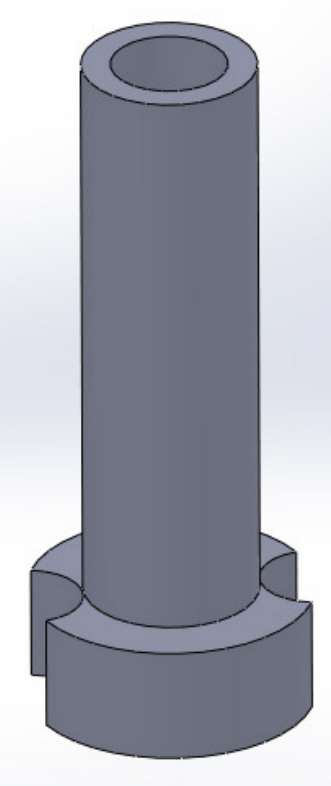

Figure 2.8: Round crucible fabricated from EDM-3 for work function testing.

The precursor powders were deposited into the crucible, which was heated in accordance to the fabrication process described in Section 2.3 in a vertical position, as shown in Figure 2.8. The resulting product was a circular, slightly concave, metalliclooking electride surface. The crucible with the electride was then inserted into a hollow cathode barrel, with the electride surface approximately $1.27 \mathrm{~mm}$ upstream of the orifice plate. The cathode was started and operated at $1.3 \mathrm{~A}$ according to the procedures described in Chapter 3 over the course of four different runs, each approximately two hours in duration.

After the hollow cathode conditioning process, the crucible was removed and placed in a diode work function apparatus, as shown in Figure 2.9 and Figure 2.10. 


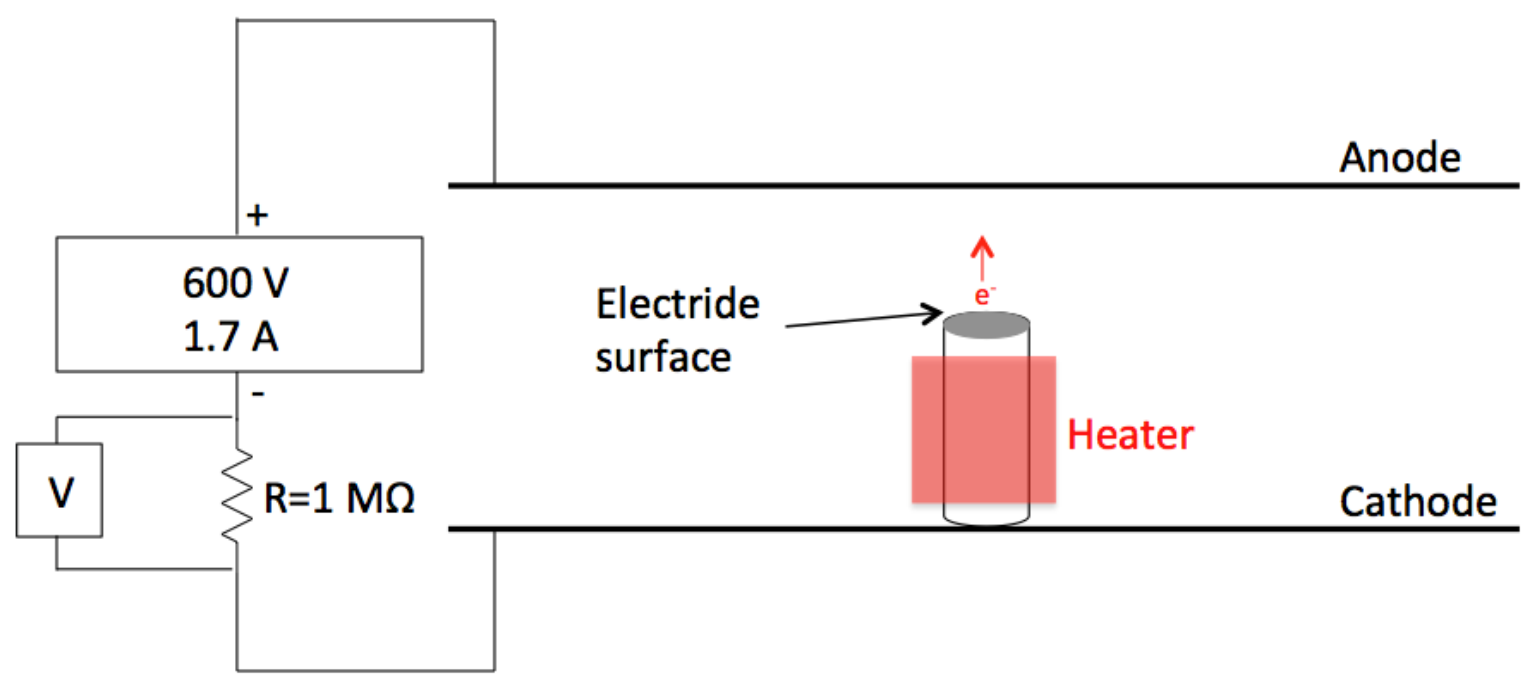

Figure 2.9: Schematic of the diode apparatus used to measure the electride work function.

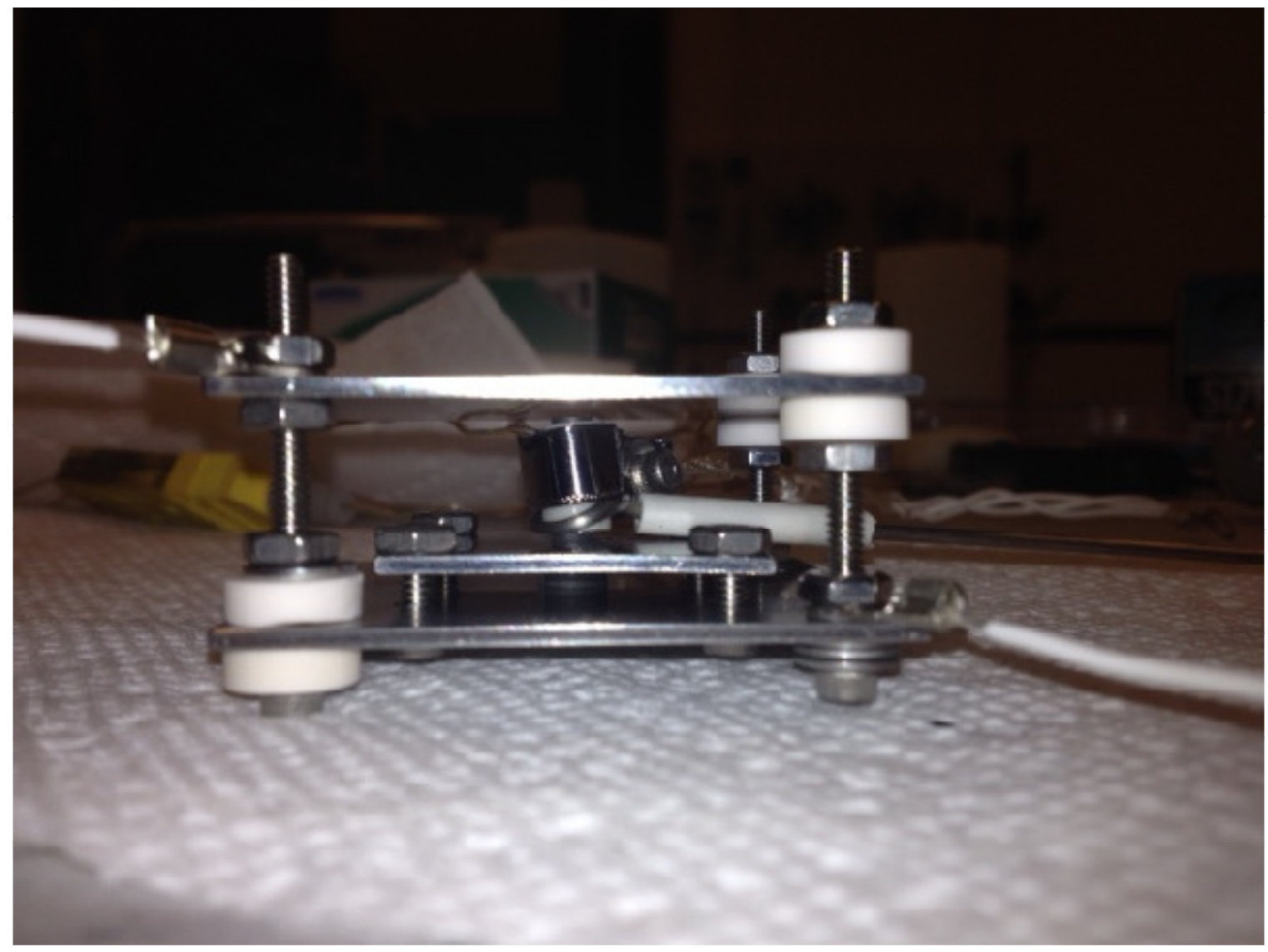

Figure 2.10: Picture of the diode apparatus used to measure the electride work function. A swaged tantalum heater was fit around the crucible, which was electrically isolated from the crucible. A positive voltage was placed on the anode plate suspended approximately 
$3.8 \mathrm{~mm}$ over the electride surface. The electric field assured that the emission was thermionically limited, as described by equation (2.1), rather than inhibited by the space charge effect [22]. The emitted current was quantified by measuring the voltage across a one-megaohm resistor placed in series with the diode apparatus, as shown in Figure 2.9. New, clean insulators were used to prevent leakage current between the anode and the cathode. The leakage current was measured with a blank crucible at elevated temperatures and was found to be below detectable limits. The sheath of the coaxial heater was kept completely isolated, to prevent any thermionic emission from the heater to the anode.

The measurement was performed according to the technique described in references [18] and [20]. The emission was measured at several different temperatures over seven different voltages ranging from zero to $600 \mathrm{~V}$. This technique is only an effective quantification of work function if the emission is not time-dependent over the duration of the voltage sweep. $0.15 \mathrm{~A} / \mathrm{m}^{2}$ of emission was observed from the CSU electride at a temperature of $50{ }^{\circ} \mathrm{C}$. However, that emission was observed to decrease to below detectable limits within 13 minutes. Several additional measurements were taken at increasing temperatures, and while measurable emission current was observed, the current never reached the intensity observed in the first test. Figure 2.11 shows the peak currents measured at $600 \mathrm{~V}$ over a range of temperatures versus time. 


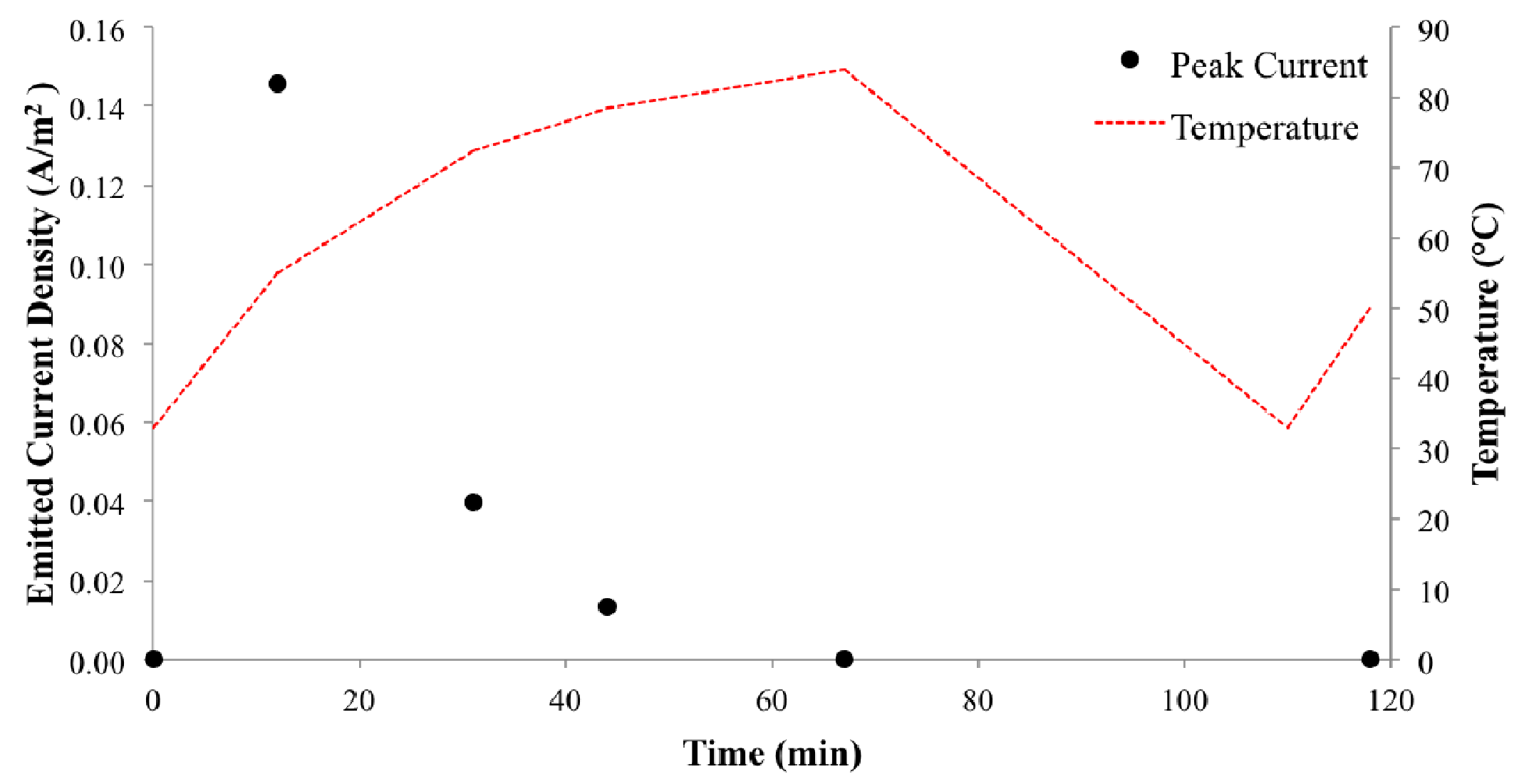

Figure 2.11: Peak current densities emitted at $600 \mathrm{~V}$ over a range of temperatures vs. time. 


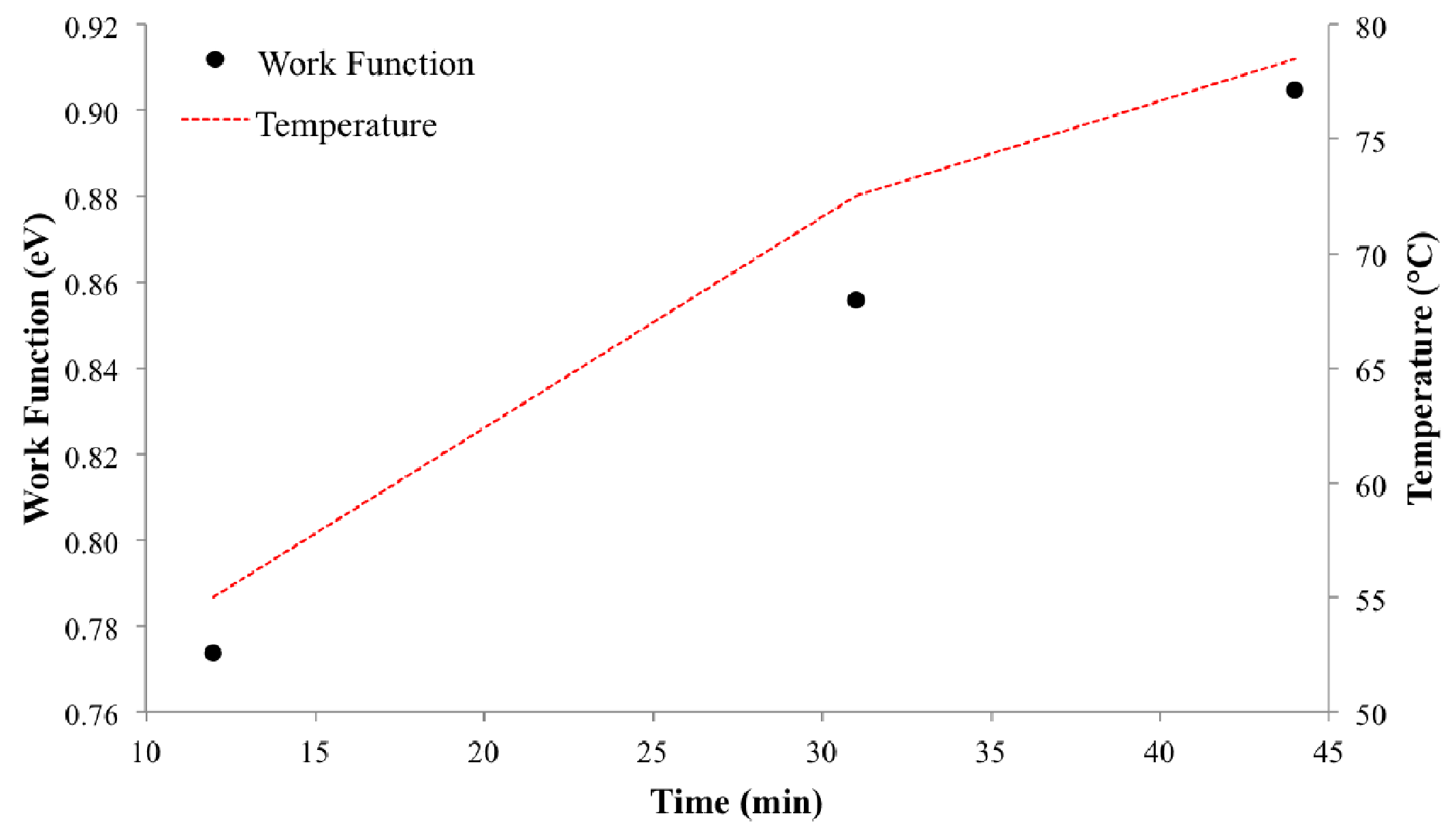

Figure 2.12: The calculated work functions from the peak measured currents at $600 \mathrm{~V}$ vs. time. 
Given the time-dependent nature of the emission, the work function could not be calculated according to Cronin's technique [20]. Instead, an estimation was made for the Sommerfeld constant $(\boldsymbol{A})$ in equation (2.1) to obtain a work function value. Using the highest observed current emission, $0.15 \mathrm{~A} / \mathrm{m}^{2}$, and assuming a value of $120 \times 10^{5} \mathrm{Am}^{-2} \mathrm{~K}^{-2}$ for the Sommerfeld constant, a work function of $0.76 \mathrm{eV}$ was calculated. Work functions were also calculated for the other peak values measured, as shown in Figure 2.12.

There are several explanations behind the apparent increase in the work function over time. The simplest of which is basic contamination. The chamber in which the work function measurement was performed was pumped via an oil-based diffusion pump that lacked a cooled baffle. It is likely that oil was introduced into the system as soon as the gate valve on the chamber was opened. If that oil coated the electride surface, it could have acted as an insulating layer, inhibiting emission.

Alternatively, the increase in work function may be indicative of a more fundamental property of the emission. Phillips et al [29] measured the thermionic emission from potassium and rubidium cryptand electride thin films. They reported the thermionic emission at temperatures between $-65^{\circ} \mathrm{C}$ and $0{ }^{\circ} \mathrm{C}$. The resulting thermionic emission was found to decrease with time, similar to the phenomenon observed in the measurements of the directly synthesized electride. Phillips et al also described that additional emission was recovered when the temperature was increased and their maximum emission current increased with increasing temperature.

Phillips et al speculated that the initial emission was a result of electrons located in electronic states near the vacuum level due to surface defects. After emission, these states were not replenished, leading to an apparent increase in work function. They believed that 
thermal decomposition of the surface (known to occur at temperatures above $-60{ }^{\circ} \mathrm{C}$ ) led to the creation of new defect sites when the temperature was increased, a notion that was supported by the increasing height of the peak emission current with temperature. If a surface depletion mechanism was indeed responsible for the increase in work function with time observed for the directly synthesized electride, then it is possible that there was no decomposition at the surface, leading to no creation of new defect sites. As a result, the only emission seen at elevated temperatures would be from electrons in states that are too far from the vacuum level to emit at lower temperatures. This is supported by the decreasing height of the peak emission amounts with temperature, as shown in Figure 2.11. Given that C12A7 electride has been reported to be stable in vacuum up to $1000{ }^{\circ} \mathrm{C}$ [30], it is unlikely that any thermal decomposition took place at the low temperatures used in the work function measurement.

The possibility that depleted surface energy states affected emission brings additional complications into how the hollow cathode operates. It is possible that electrons from the surface defect states are enough to start the cathode (discussed more in Chapter 4), after which point the cathode is hot enough that it can operate on electron emission from the bulk. It may be that the bulk work function is the $2.4 \mathrm{eV}$ quantity measured by Toda et al [9]. At some point, whether it is during the cathode operation of the cooling process after the cathode has been turned off, perhaps the surface defect states repopulate. Additional work function measurements using directly synthesized electride samples that had not been operated in a cathode were unable to detect any emission current, indicating that the samples had high work functions. It is possible that the light ion bombardment at the 5-10 eV levels found within a hollow cathode continuously repopulates the surface 
defect sites. An additional investigation into the emission mechanism of C12A7 electride beyond what is discussed herein is certainly warranted. 


\section{References}

[1] C. a. Kraus, J. Am. Chem. Soc. 29, 1557 (1907).

[2] J. L. Dye, Science (80-. ). 301, 607 (2003).

[3] R. Eisberg and R. Resnick, Quantum Physics of Atoms, Molecules, Solids, Nuclei, and Particles, Second (John Wiley \& Sons Inc, New York, NY, 1985).

[4] J. Dye, Science (80-. ). 247, 663 (1990).

[5] C. Pedersen, J. Am. Chem. Soc. 89, 7017 (1967).

[6] J. Lehn, Acc. Chem. Res. 174, 49 (1978).

[7] S. Matsuishi, Y. Toda, M. Miyakawa, K. Hayashi, T. Kamiya, M. Hirano, I. Tanaka, and H. Hosono, Science 301, 626 (2003).

[8] H. Hosono, S. Kim, M. Miyakawa, S. Matsuishi, and T. Kamiya, J. Non. Cryst. Solids 354, 2772 (2008).

[9] Y. Toda, H. Yanagi, E. Ikenaga, J. J. Kim, M. Kobata, S. Ueda, T. Kamiya, M. Hirano, K. Kobayashi, and H. Hosono, Adv. Mater. 19, 3564 (2007).

[10] P. Sushko and A. Shluger, J. Am. ... 129, 942 (2007).

[11] D. A. Neamen, Semiconductor Physics and Devices, Fourth (McGraw-Hill, New York, NY, 2012).

[12] M. Brustolon and G. Giamello, Electron Paramagnetic Resonance (Wiley, Hoboken, New Jersey, 2009).

[13] B. Odom, D. Hanneke, B. D’Urso, and G. Gabrielse, Phys. Rev. Lett. 97, 6 (2006).

[14] W. Rice, R. Weber, and A. Leonard, Lasers Electro- ... 5 (2011).

[15] Y. Toda, Y. Kubota, M. Hirano, H. Hirayama, and H. Hosono, ACS Nano 5, 1907 (2011).

[16] S. Kim, M. Miyakawa, K. Hayashi, T. Sakai, M. Hirano, and H. Hosono, J. Am. Chem. Soc. 127, 1370 (2005).

[17] S. Kim, S. Matsuishi, M. Miyakawa, K. Hayashi, M. Hirano, and H. Hosono, J. Mater. Sci. Mater. Electron. 18, 5 (2007). 
[18] S. Kim, Y. Toda, K. Hayashi, M. Hirano, and H. Hosono, Chem. Mater. 18, 1938 (2006).

[19] S. W. Kim, T. Shimoyama, and H. Hosono, Science 333, 71 (2011).

[20] J. Cronin, IEE Proc. I (Solid-State Electron Devices) 128, (1981).

[21] Y. Toda, S. Kim, K. Hayashi, M. Hirano, T. Kamiya, H. Hosono, T. Haraguchi, and H. Yasuda, Appl. Phys. Lett. 87, 254103 (2005).

[22] K. L. Jensen, Electron Emission Physics, Advances i (Elsevier Inc., San Diego, California, 2007).

[23] R. Schlaf, H. Murata, and Z. . Kafafi, J. Electron Spectros. Relat. Phenomena 120, 149 (2001).

[24] S. Miyazaki, T. Maruyama, A. Kohno, and M. Hirose, Microelectron. Eng. 48, 63 (1999).

[25] Ohio Carbon Blank, (2011).

[26] Y. Waseda, E. Matsubara, and K. Shinoda, X-Ray Diffraction and Crystallography Introduction, Examples and Solved Problems (Springer, Berlin, Germany, 2011).

[27] J. F. Watts, An Introduction to Surface Analysis by XPS and AES (J. Wiley, Chichester, West Sussex, England, 2003).

[28] A. V. Naumkin, A. Kraut-Vass, S. W. Gaarenstroom, and C. J. Powell, NIST Stand. Ref. Database 20, Version 4.1 (2012).

[29] R. Phillips, W. Pratt, and J. Dye, Chem. Mater. 12, 3642 (2000).

[30] Y. Toda, S. Matsuishi, K. Hayashi, K. Ueda, T. Kamiya, M. Hirano, and H. Hosono, Adv. Mater. 16, 685 (2004). 


\section{CATHODE FABRICATION AND APPARATUS}

This chapter describes the methods employed to assemble a cathode using the electride described in Chapter 2. Additionally, the experimental configuration used to test the cathode performance is discussed.

\subsection{Test Facility}

The cathode testing was conducted in a $1.7 \mathrm{~m}$ diameter, $4.6 \mathrm{~m}$ long steel vacuum chamber. The chamber was diffusion pumped, with a base pressure of approximately $6 \times 10^{-6}$ Torr after five hours of pump operation. The chamber pressure was $2 \times 10^{-5}$ Torr at 4 sccm of xenon, a common mass flow rate used to test the hollow cathodes.

\section{$3.2 \quad$ Test Apparatus}

A diode configuration was used to evaluate cathode performance, as shown in Figure 3.1. A Xantrex XFR 100-12 DC Power Supply was utilized as the discharge power supply, driving the circuit between the cathode and the anode. The keeper power supply was a Spellman SL1200. A ring anode was fabricated from a piece of stainless steel shim stock that was $0.38 \mathrm{~mm}$ thick, $5 \mathrm{~cm}$ wide, and $24 \mathrm{~cm}$ long. The shim stock was rolled into a ring $7.6 \mathrm{~cm}$ in diameter and mounted along the axis of cathode orifice with its upstream edge $3 \mathrm{~cm}$ downstream of the exit plane. A photograph of the open testing apparatus, including the anode, is shown in Figure 3.2.

For the majority of cathode evaluation, an open wire keeper was used. The reasons for this were two-fold. A thermocouple could be easily mounted on the barrel to measure operating temperature, and the cathode operation was easy to observe. The thermocouple was a type $\mathrm{R}$ thermocouple with one layer of $0.0254 \mathrm{~mm}$ thick tantalum foil placed over the 


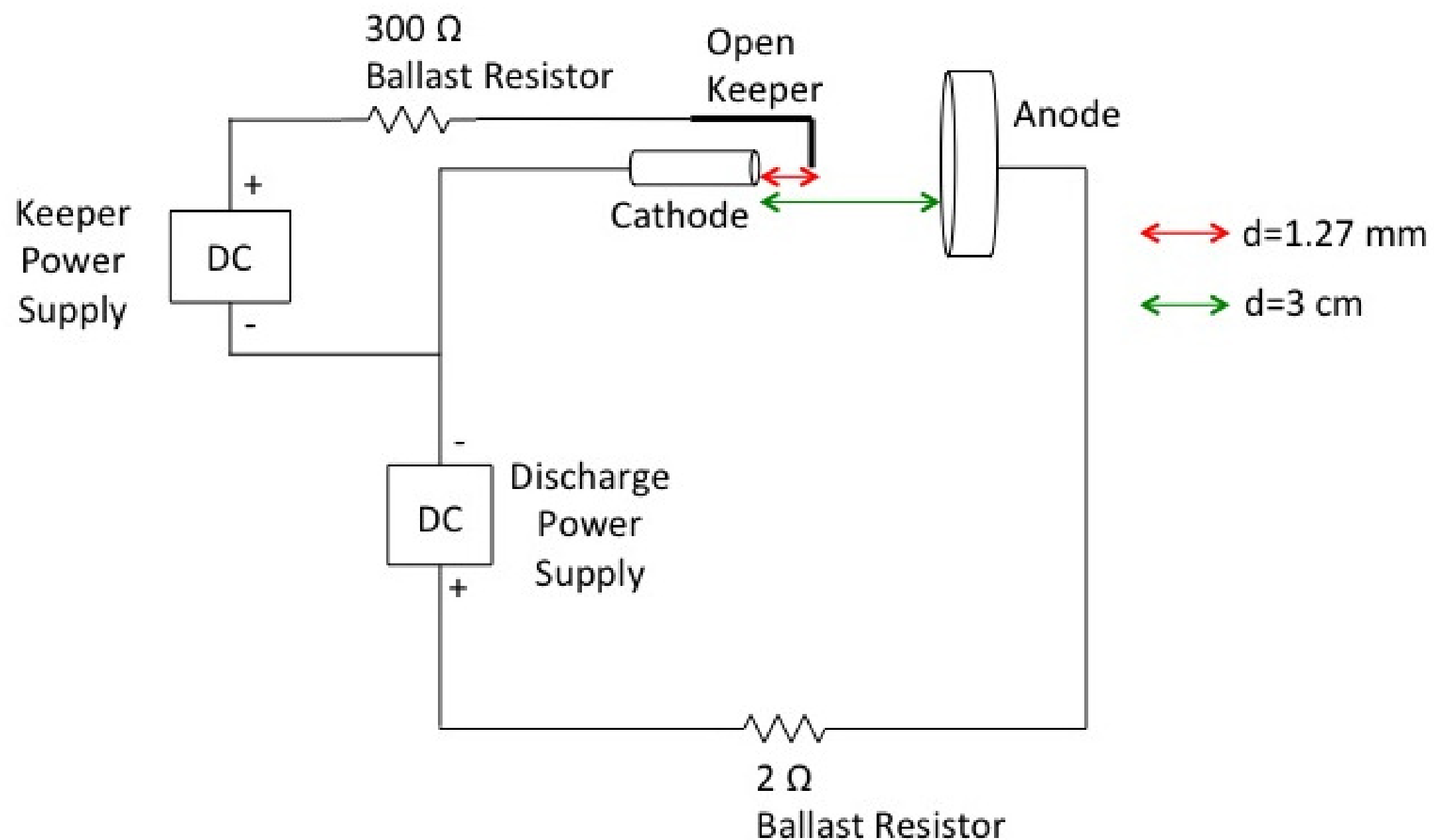

Figure 3.1: Circuit diagram of diode configuration test apparatus. 
junction. The thermocouple junction was spot welded to the downstream end of the cathode barrel where it joined the orifice plate. For some tests, an enclosed graphite keeper was utilized, which did not allow the use of a thermocouple.

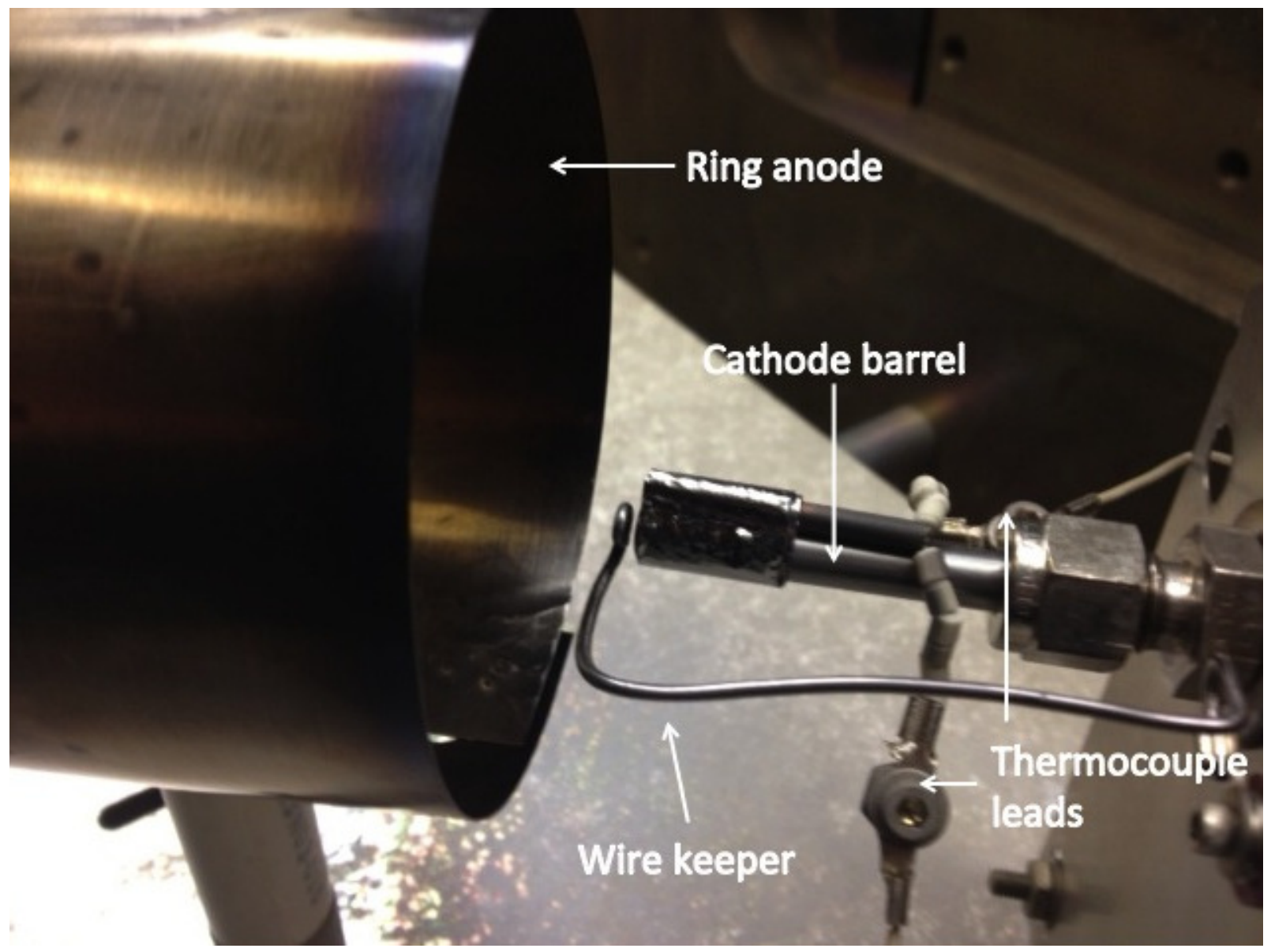

Figure 3.2: Cathode testing apparatus.

\subsection{Barrel Material}

The initial electride hollow cathode prototype utilized a $1.27 \mathrm{~cm}$ diameter graphite barrel, fabricated from EDM-3 grade graphite supplied by Poco Graphite [1]. Graphite was chosen because the electride could be melted directly on the inner surface of the barrel. The precursors were mixed and put directly in the tube, and the entire tube was then placed in the vacuum furnace and heated according to the procedure described in Chapter 
2. The resulting cathode typically had several solidified electride droplets stuck to the graphite at uncontrollable intervals down the barrel. The non-continuous nature of the emission surface was not ideal. Additionally, the electride droplet furthest downstream was often as much as $1.27 \mathrm{~cm}$ upstream of the orifice plate. Given that phenomenological models predict the majority of electron emission in a hollow cathode will occur in the first few millimeters of the insert [2], the relatively far upstream position of the electride was also not ideal.

The resulting operation was unstable with periodic material ejection and frequent "wink outs", in which the discharge would extinguish and have to be re-started. During operation, the anode voltage varied considerably. It is thought that the variation may have been due, in part, to the dominant emission site changing between the multiple electride droplets. Another cause of the unstable operation was the graphite orifice plate. Graphite is highly susceptible to arcing, a result of which produces pitting in the surface [3]. As the keeper voltages spiked when the cathode winked out, arcing between the orifice plate and keeper resulted in the buildup of material that partially occluded the orifice in some tests. The visible ejection of sparks occurred periodically during operation, as shown in the photograph in Figure 3.3.

To improve the stability of the cathode and decrease arcing, a $0.635 \mathrm{~cm}$ diameter tantalum tube was used in place of the graphite barrel. The tantalum barrel was capped with a thoriated tungsten orifice plate with an orifice diameter of $0.76 \mathrm{~mm}$. Rather than melt the electride precursors directly onto the inner surface of the barrel, the precursors were heated in a graphite crucible. The resulting electride was broken into pieces and 


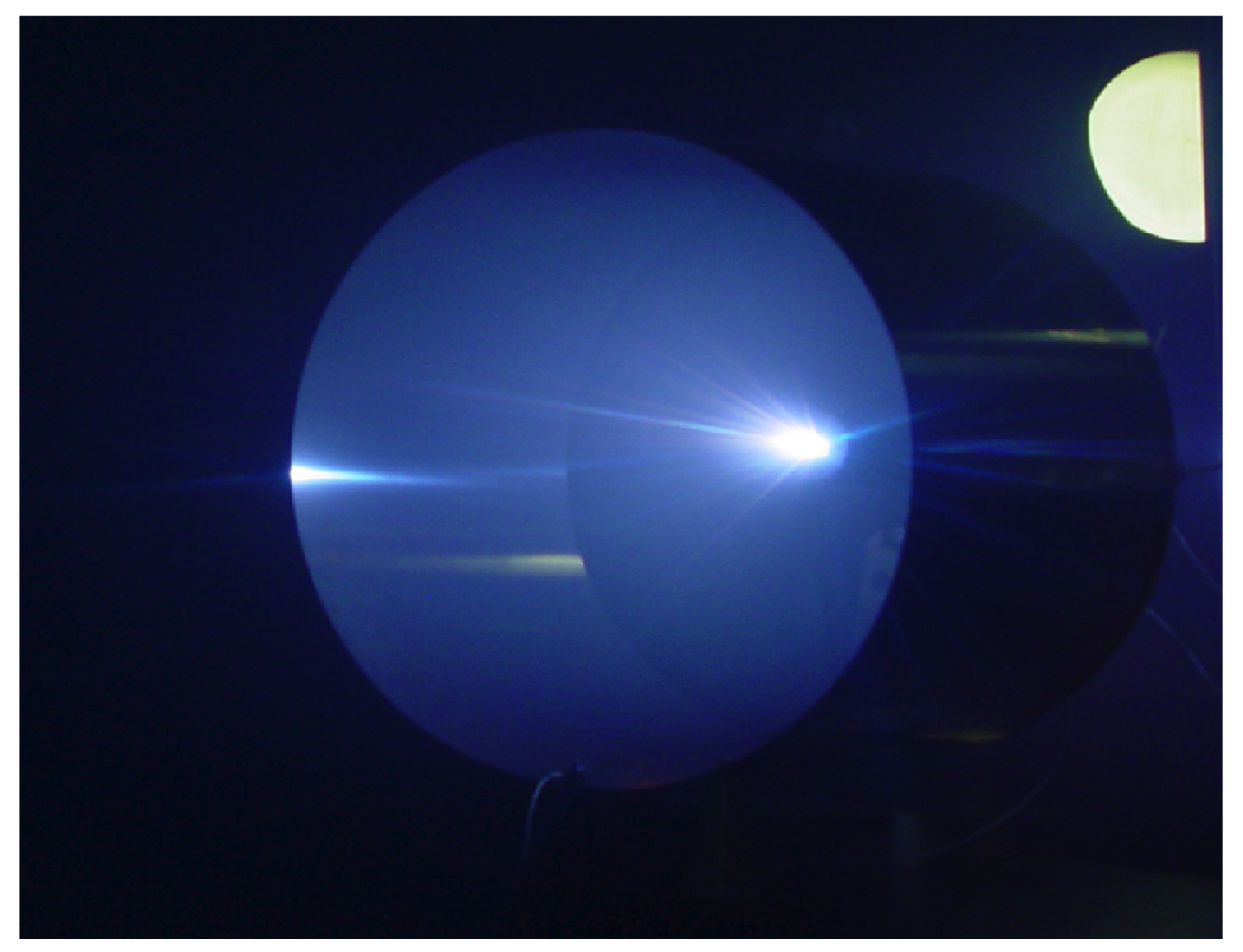

Figure 3.3: Electride hollow cathode operating unstably with material ejection. The cathode was fabricated from graphite.

several pieces were inserted into the orificed tantalum tube. The resulting cathode did not "wink-out" over the course of multiple hours of operation, which was a substantial improvement over cathode operation with the graphite barrel. It ran for over seven hours without winking out, although the operation was highly unstable. This instability was typified by large fluctuations in anode voltage and the occasional visible ejection of material. It is thought that the movement of emission sites between the different electride pieces could cause the observed instability in the anode voltage. Differing dominant sources of current would be reflected in the anode voltage because emission from a piece further upstream in the barrel would require higher voltage. 


\subsection{Insert Fabrication}

A reliable and reproducible fabrication process was developed to form inserts from electride melted in a rectangular graphite crucible, like the one shown in Figure 3.4.

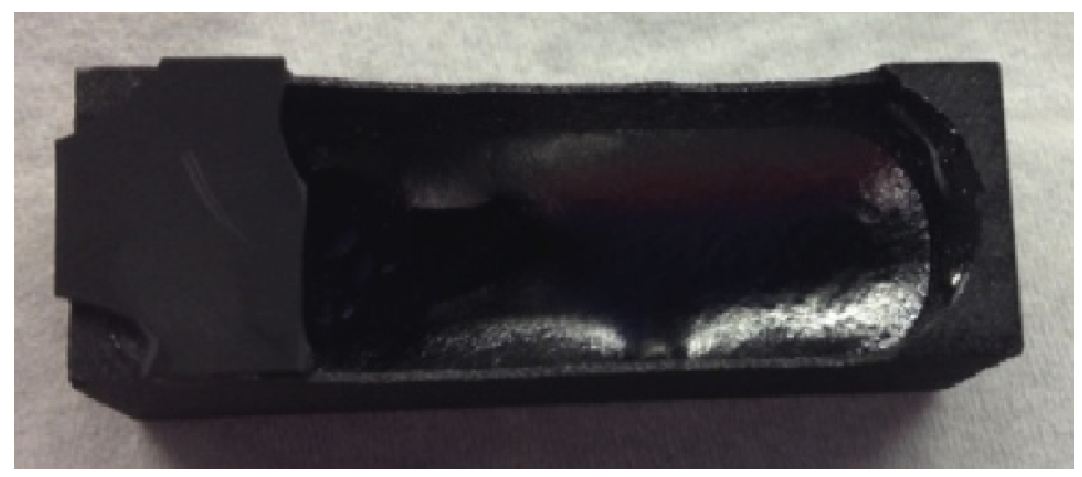

Figure 3.4: Graphite crucible used to produce electride. The electride is the metallic material in the center cavity.

A diamond-coated Dremel blade was used to separate rectangular sections of the crucible. The resulting pieces were approximately $1.9 \mathrm{~mm}$ wide and $12.7 \mathrm{~mm}$ long. The flat face of the blade was then used to grind the crucible graphite from the electride, leaving a sliver consisting almost entirely of electride. An example of a sliver is shown in Figure 3.5, and its operation in Figure 3.6.

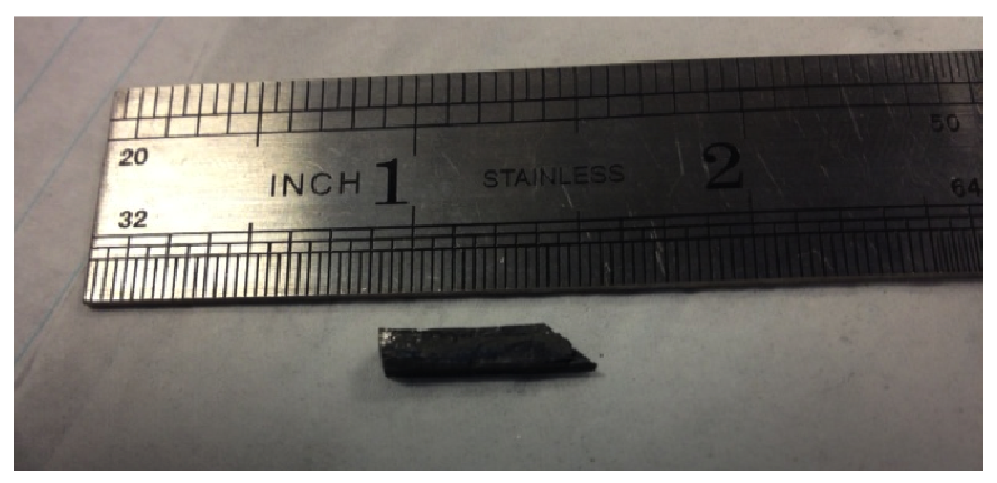

Figure 3.5: Electride sliver cut from crucible using diamond-coated Dremel blade. 


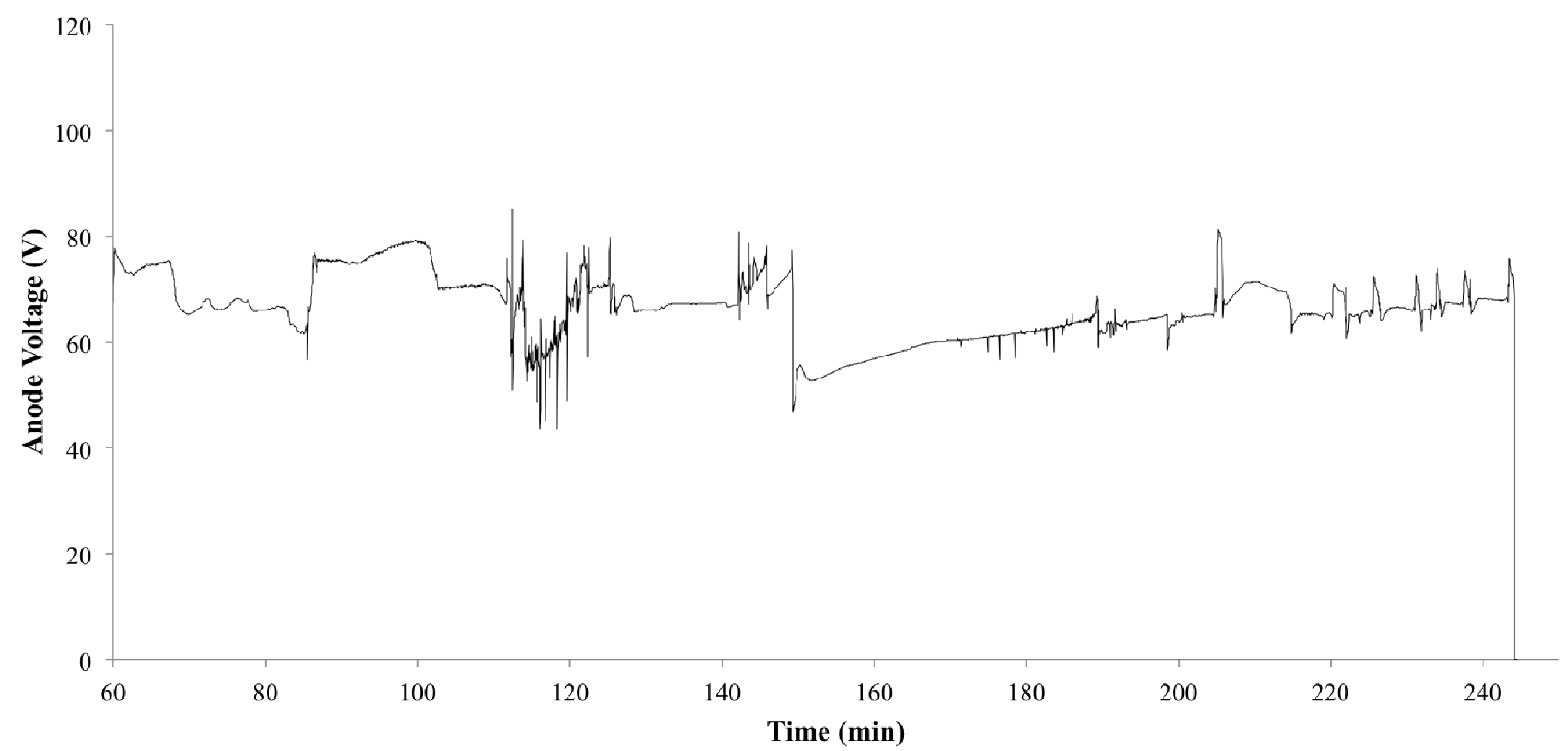

Figure 3.6: Anode voltage as a function of time for an electride hollow cathode with an insert formed from a continuous electride sliver. 
To keep the electride in its conductive form, an anionic template such as finegrained graphite needed to be present in the hollow cathode barrel. An orificed graphite tube fabricated from EDM-3 graphite at Ohio Carbon Blank, Inc. was created [6], as shown in Figure 3.7.

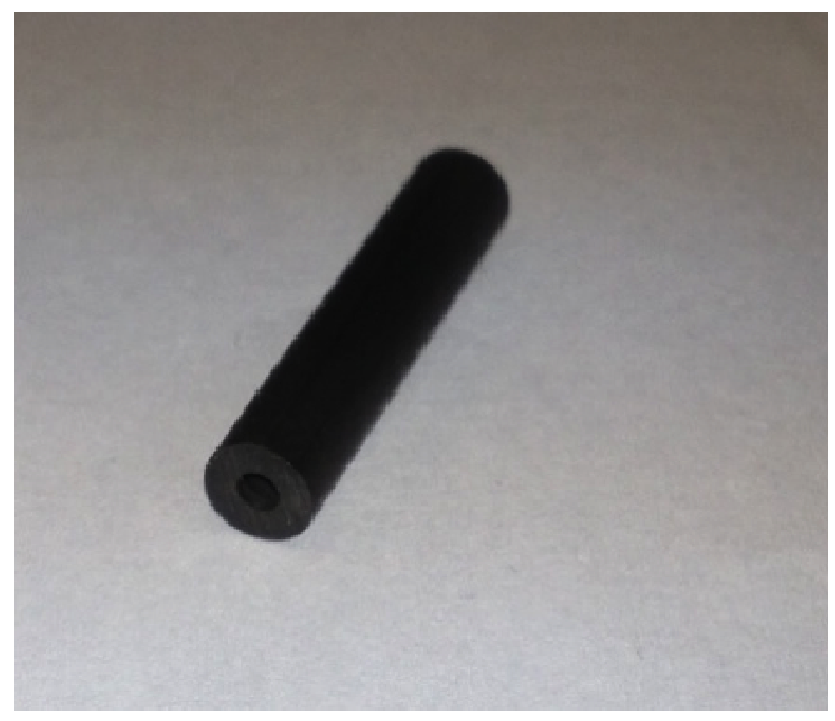

Figure 3.7: Graphite sleeve fabricated from EDM-3.

The tube was $2.54 \mathrm{~cm}$ long with an inner diameter of $2.54 \mathrm{~mm}$ and an orifice diameter of $1.9 \mathrm{~mm}$. It was designed to be "slip-fit" in the tantalum barrel.

\subsection{Insert Preparation}

Once the graphite sleeve was incorporated into the cathode design, the electride hollow cathodes could be restarted multiple times similar to the traditional $\mathrm{LaB} 6$ and $\mathrm{BaO}$ W cathodes. However, it was observed that multiple runs of several hours each were required before a cathode reached reproducible operation. Reproducible operation was important to achieve because it allowed for an investigation of orifice diameter to be conducted. 
In this context, reproducible operation is defined as duplicated anode voltages and barrel temperatures at a given set point. Even stable traditional hollow cathodes will exhibit slightly different anode voltages and operating temperatures between operational periods. This is primarily due to external factors such as the coating of the anode surface and slightly different background pressures or mass flow rates. As a result, defining reproducible operation to the tenth of a volt or single degree Celsius was unrealistic. For the following data, reproducible operation was defined as an anode voltage constant and repeatable within three volts and an operating temperature constant and repeatable within $50{ }^{\circ} \mathrm{C}$.

In general, it was found that the first two or three times an insert was run, the cathode generally exhibited initially high and decreasing anode voltages and barrel temperatures. After three or four runs, the anode voltages and barrel temperatures at different set points became constant. Figure 3.8 and Figure 3.9 show this progression for a single insert over the course of four runs. The operation is deemed reproducible between the third and fourth run, due to the convergence in voltage and temperature.

The reason behind the preparation process is unknown. The sliver manufacturing process described in section 3.4 resulted in a large quantity of graphite dust. Additional sources of contamination could exist simply from the handling that occurred during the manufacturing process. No stringent cleaning procedures were used between the sliver cutting and the use of the piece as an emitter. As a result, it is possible that a contamination layer exists on the surface. The changes that occur during the preparation process, namely the decreasing anode voltage and temperature, indicate that the insert is conditioning due to some sort of cleaning that is taking place or due to some transformation of the electride 


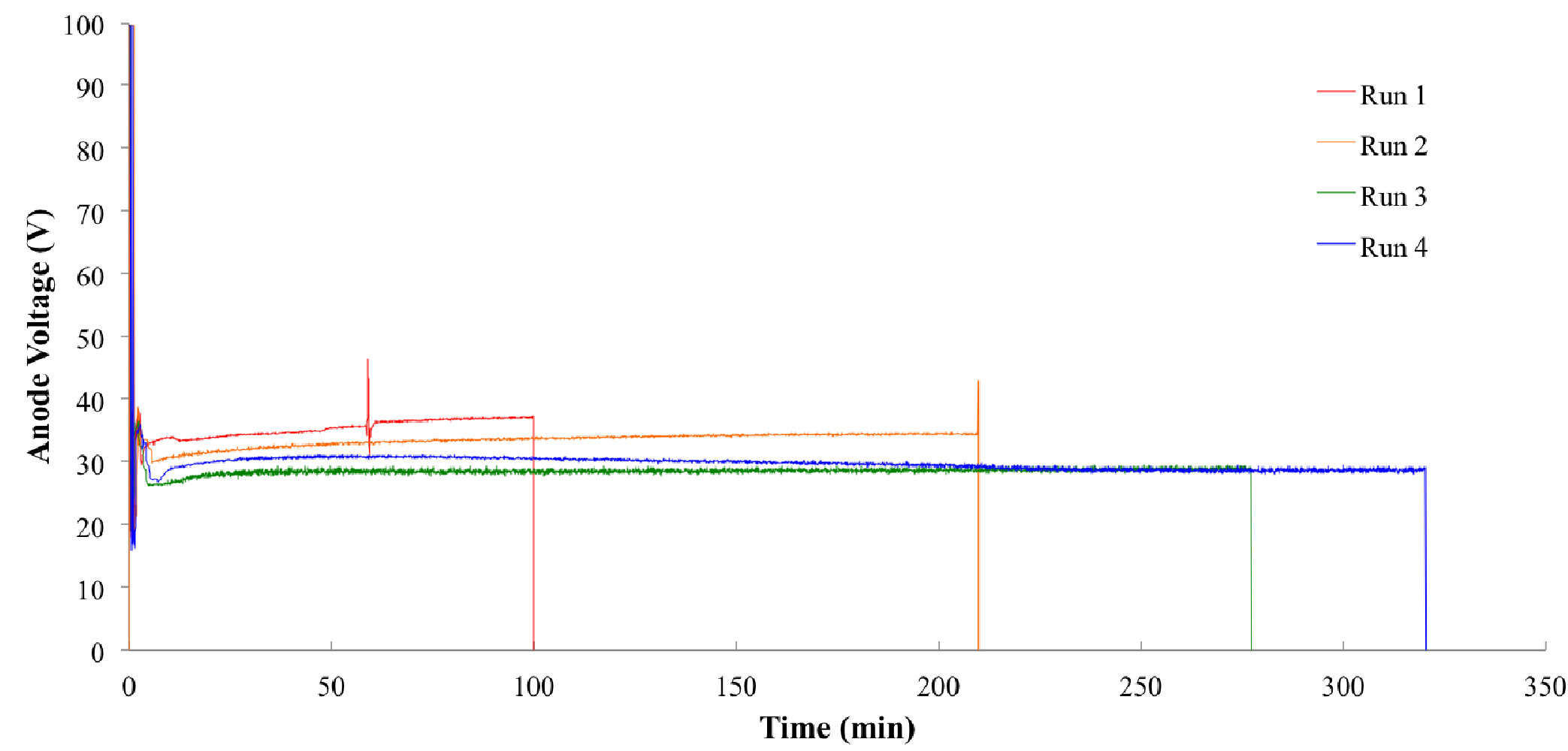

Figure 3.8: Anode voltage as a function of time over the course of four runs during the preparation of an insert. Runs 3 and 4 are deemed close enough to indicate reproducibility. 


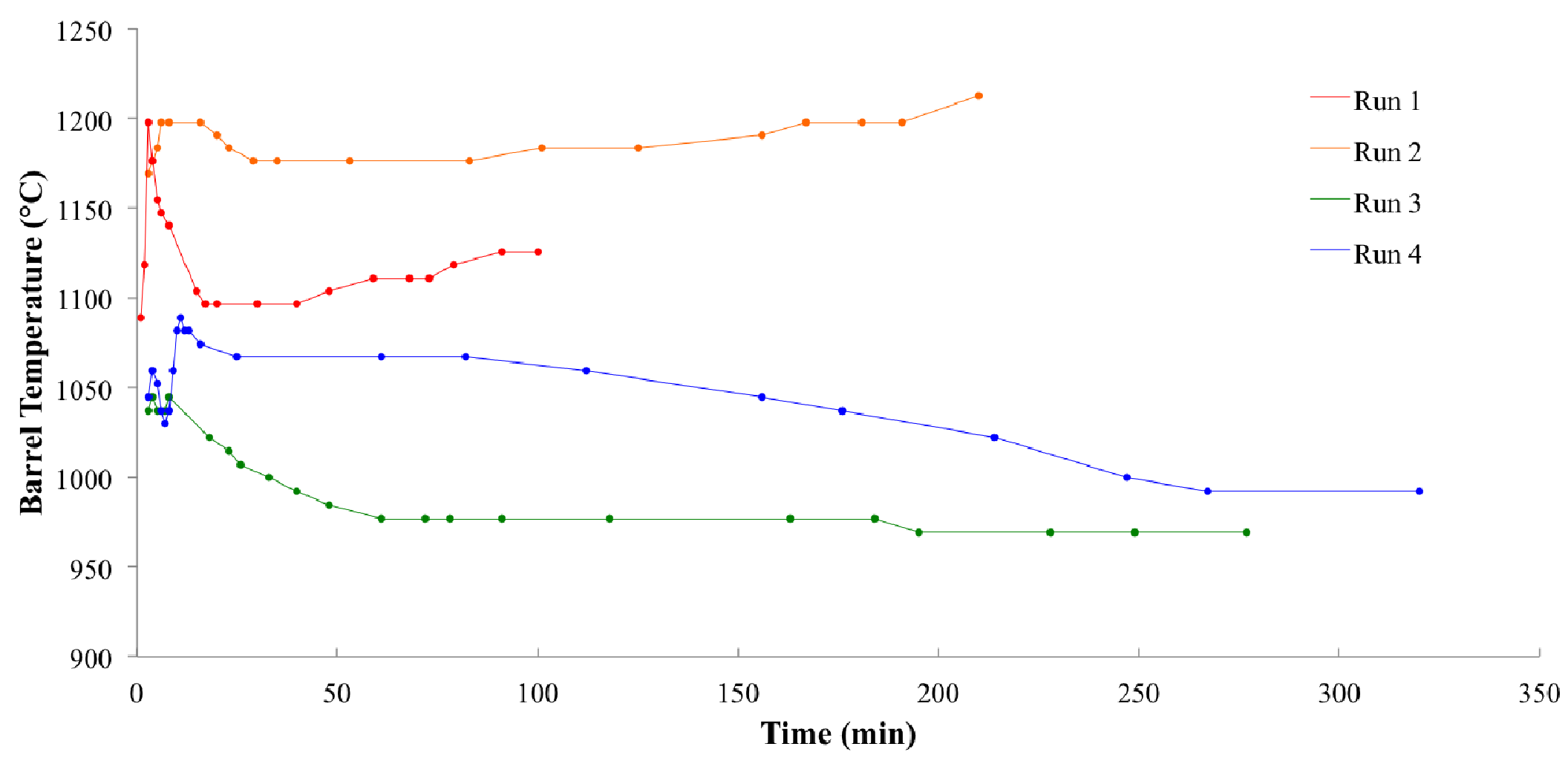

Figure 3.9: Barrel temperature as a function of time over the course of four runs during the preparation of an insert. Runs 3 and 4 are deemed close enough to indicate reproducibility. 
surface with time. It is noted that switching the cathode off was required before an improvement in temperature and anode voltage occurred, so a quenching process of heat treatment may also play a role in conditioning the electride. 


\section{References}

[1] Poco Graphite, (2013).

[2] D. E. Siegfried, A Phenomenological Model for Orificed Hollow Cathodes, Colorado State University, 1983.

[3] D. Goebel, in 41st AIAA/ASME/SAE/ ASEE Jt. Propuls. Conf. Exhib. (2005).

[4] S. Kim, Y. Toda, K. Hayashi, M. Hirano, and H. Hosono, Chem. Mater. 18, 1938 (2006).

[5] S. Kim, Y. Toda, K. Hayashi, M. Hirano, and H. Hosono, Chem. Mater. 18, 1938 (2006).

[6] Ohio Carbon Blank, (2011). 


\section{CATHODE PERFORMANCE}

This chapter characterizes the operation of an electride hollow cathode. The cathode consists of a tantalum barrel, graphite liner, and continuous insert surface, as described in Section 3.4. Testing of an initial prototype design in conjunction with a Hall thruster is discussed in Appendix A.

\subsection{Starting Characteristics and Protocol}

Relatively low voltage on the keeper was required to start the electride cathode and there was no visible arc activity detected. The cathode did not appear to need an arcing event to initiate operation, and the voltage applied to the keeper was not adequate to cause arcing. According to Raizer [4], the breakdown voltage, $V_{B}$ at which an arc occurs is

$$
V_{B}=\frac{B p d}{\ln \left(\frac{G p d}{\ln \left(1+\frac{1}{\gamma}\right)}\right)}
$$

where $B$ and $G$ are constants specific to xenon, $\gamma$ is a constant specific to the surface material, $p$ is the pressure and $d$ is the distance between the electrodes. Equation 4.1 is applicable for all $p d>(p d)_{l i m}$, where $(p d)_{\text {lim }}$ can be found according to

$$
(p d)_{\lim }=G^{-1} \ln \left(\frac{1}{\gamma}+1\right)
$$

At $(p d)_{l i m}$, the amplification of single ionization events caused by random occurrences such as cosmic rays is not sufficient to start the avalanche effect that creates a breakdown. For a 
breakdown to have occurred to start the cathode, the required voltage at the relevant $p d$ needed to be greater than $V_{B}$ and $p d$ must be shown to have been greater than $(p d)_{\text {lim. }}$.

For the following discussion, the keeper and orifice plate are treated as parallel plates separated by a distance $d$ of $0.127 \mathrm{~cm}$. At $25 \mathrm{sccm}$ of xenon flow, the background pressure in the vacuum chamber used for cathode testing was $\sim 1 \times 10^{-4}$ Torr. To estimate the pressure between the orifice plate and keeper before the cathode started, the $25 \mathrm{sccm}$ of xenon was assumed to emit from a point-like source located at the orifice exit plane. A sphere was drawn with a radius $d$ centered at the point source and extending to the keeper. The xenon atoms were assumed to move radially-outward from the point and subsequently expand uniformly. Thus, it was possible to find the pressure at any distance away from the point source by calculating the number of particles in the surface area on the sphere specified by a solid angle. For this discussion, the solid angle was assumed to be $\pi$ steradians, as shown Figure 4.1.

The surface area of $A$ as shown in Figure 4.1 was $1 / 4$ the surface area of the sphere. With $d$ specified as $0.13 \mathrm{~cm}, A$ was calculated to be $5 \times 10^{-6} \mathrm{~m}^{2}$. The number density at the keeper was found using the area $A$, the atomic flow rate $\dot{N}$, and the thermal velocity $v_{t h}$, according to equation 4.3 .

$$
n=\frac{\dot{N}}{A v_{t h}}
$$

The atomic flow rate for $25 \mathrm{sccm}$ of xenon is $1.1 \times 10^{19}$ atoms/s. The thermal velocity is denoted by equation 4.4 as 


\section{Cathode}

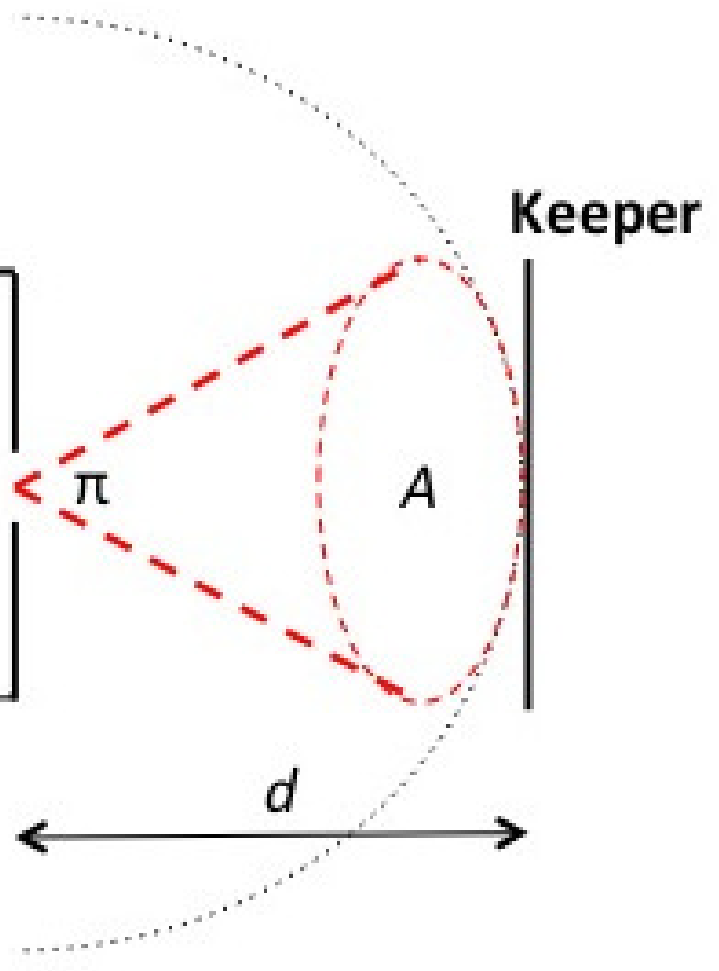

Figure 4.1: The geometry used to calculate the breakdown voltage between the cathode orifice plate and the keeper. The mass flow rate is assumed to originate at a point source centered in the orifice and expand outward at a solid angle of $\pi \mathrm{sr}$.

$$
v_{t h}=\sqrt{\frac{8 k T}{\pi m}}
$$

The number density $n$ was found to be $1 \times 10^{22}$ particles per square meter. The pressure could then be found with the ideal gas law

$$
P=n k T
$$

The $P$ used in equation 4.5 was calculated midway between the orifice and the keeper. The actual pressure, found by summing the calculated $P$ and the background pressure of the chamber, was estimated to be 0.3 Torr. The resulting $p d$ was found to be 0.038 Torr-cm. The minimum quantity for a breakdown to occur, $p d_{l i m}$, is 0.11 Torr-cm. As a result, it was 
concluded that the start-up mechanism of the electride cathode was not likely due to the breakdown of the xenon between the keeper and the orifice plate.

It is possible that the actual start-up mechanism was similar to that observed in LaB6 cathodes. Typically, the LaB6 insert is heated until electrons are emitted, usually at approximately $1600^{\circ} \mathrm{C}[3]$. The electric field formed between the keeper and the cathode penetrates, albeit poorly, through the orifice into the insert region, accelerating the electrons downstream [5]. The electrons subsequently collide with neutral gas particles and act as triggers to the avalanche effect that results in the formation of a plasma and the ignition of the cathode [4]. These few seed electrons effectively replace the cosmic ray events needed to trigger a breakdown described by equation 4.1 , which negates the need for $p d$ to be greater than $p d_{\text {lim. }}$. Additionally, the electrons, although low in number, are much more plentiful than cosmic ray occurrences, so the discharge initiates at much lower pressures. We judge that LaB6, with a work function of $2.67 \mathrm{eV}$ [5], needs to be heated to $1600{ }^{\circ} \mathrm{C}$ to emit sufficient electrons to initiate the avalanche effect at reasonable flow rates. In comparison, electride, which has a lower work function, appears to emit a sufficient number of electrons at room temperature to trigger the initiation sequence.

There were two procedures by which the electride cathode could be started. One involved setting the mass flow rate and increasing the keeper voltage until a discharge was initiated. With $50 \mathrm{sccm}$ of xenon flowing, the cathode typically started with $400 \mathrm{~V}$ on the keeper. Alternatively, a high voltage could be applied to the keeper while the mass flow rate was increased until the cathode started. With $1000 \mathrm{~V}$ on the keeper, the cathode started with approximately $25 \mathrm{sccm}$ of xenon. Both procedures utilized the avalanche mechanism described above. Using a high mass flow rate as discussed in the fist method 
increased the number of collisions the electrons accelerated from the insert region underwent, and, as the the voltage was increased, they eventually were given enough kinetic energy to cause a sufficient number of ionization events to start the avalanche effect. In comparison, starting with a high voltage on the keeper pulled the electrons out of the insert region with a constant, relatively high kinetic energy, and, as the gas flow was increased, a critical point was reached where there were enough collisions to initiate the avalanche effect. The later procedure was used more frequently to conserve propellant. It should be noted that an attempt was made using the same start-up procedure to start an identical cathode lacking the electride insert, and a discharge could not be started nor maintained.

\subsection{Performance of the Electride Cathode}

A single insert, fabricated according to the procedure described in Section 3.4, was operated for over 60 hours with no sign of degradation. The insert was operated in three tantalum barrels of differing orifice diameters over the testing period. The testing occurred over two months and included 20 cathode restarts, 11 chamber vent-pump down sequences, and an iodine exposure in which $0.1 \mathrm{~g}$ of iodine was flowed through the tantalum barrel at room temperature. 


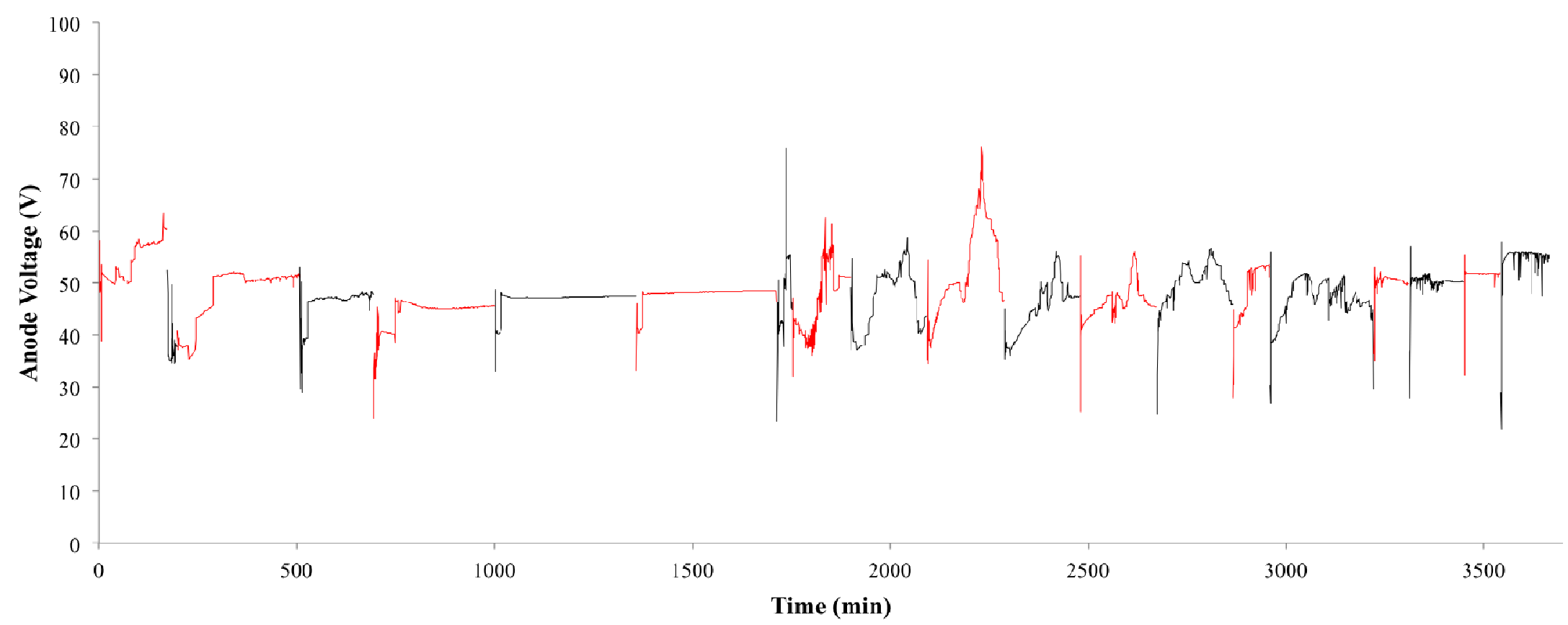

Figure 4.2: A graph of the anode voltage versus time for over 60 hours of operation on a single insert. Separate days are denoted by a change in color. Deviations in the anode voltage are due primarily to the variations of mass flow rate, discharge current, and orifice size. 


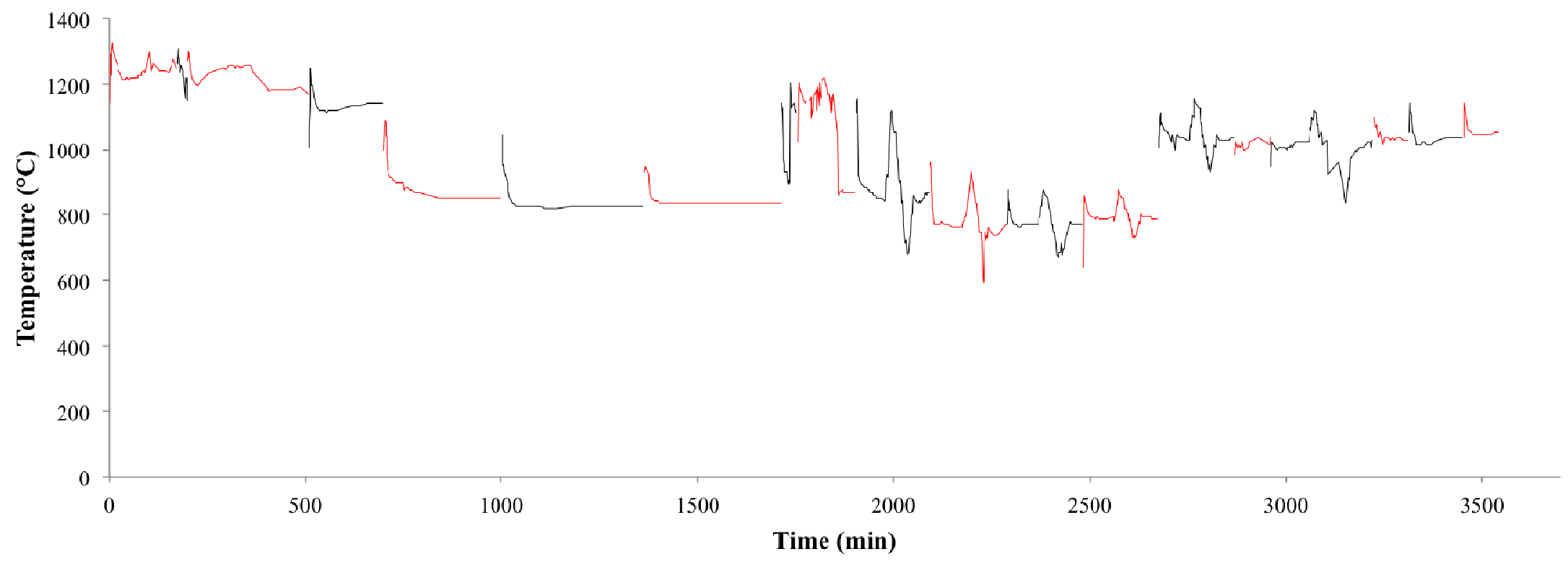

Figure 4.3: A graph of the barrel temperature time for over 60 hours of operation on a single insert. Separate days are denoted by a change in color. Deviations in the temperature are due primarily to the variations of mass flow rate, discharge current, and orifice size. 
The transition between insert conditioning and hollow cathode characterization is visible in Figure 4.2 and Figure 4.3. Between 0 and $1200 \mathrm{~min}$, the insert was operated at a constant discharge current at a fixed mass flow rate until repeatable anode voltages and barrel temperatures were observed. As a result, these sections of the graphs are relatively constant. In comparison, after $1200 \mathrm{~min}$, the mass flow rate, discharge current, and orifice size were all varied to observe their effects on anode voltage and barrel temperature. As a result, the anode voltage and temperature vary quite a bit after $1200 \mathrm{~min}$.

\subsection{Orifice Sizing}

Using the fully conditioned insert described in Section 4.2, three different orifice sizes were tested under identical conditions. The three diameters were $0.76 \mathrm{~mm}, 1.42 \mathrm{~mm}$, and $2.03 \mathrm{~mm}$. During each test, the barrel temperature was measured at a variety of mass flow rates and discharge currents. The characterization was done in the diode test configuration using an external wire keeper, which collected a constant $0.3 \mathrm{~A}$ of current that was added to the discharge current.

As shown in Figure 4.4 and Figure 4.5 , the cathode with the $0.76 \mathrm{~mm}$ orifice generally operated approximately $100^{\circ} \mathrm{C}$ hotter than the cathodes with the larger orifices. Interestingly, there did not appear to be a dramatic difference in operating temperatures between the cathode with the $1.42 \mathrm{~mm}$ orifice and the cathode with the 2.03 orifice. We judge that there could be two explanations for the increase in temperature between the $0.76 \mathrm{~mm}$ orifice and the $1.42 \mathrm{~mm}$ orifice. One possibility is that orifice heating ceased to be the primary contributing factor to the operating temperature. Since every other variable was kept constant, it is then not surprising that significant temperature changes were not observed between the two larger orificed cathodes. Alternatively, as the orifice diameter 


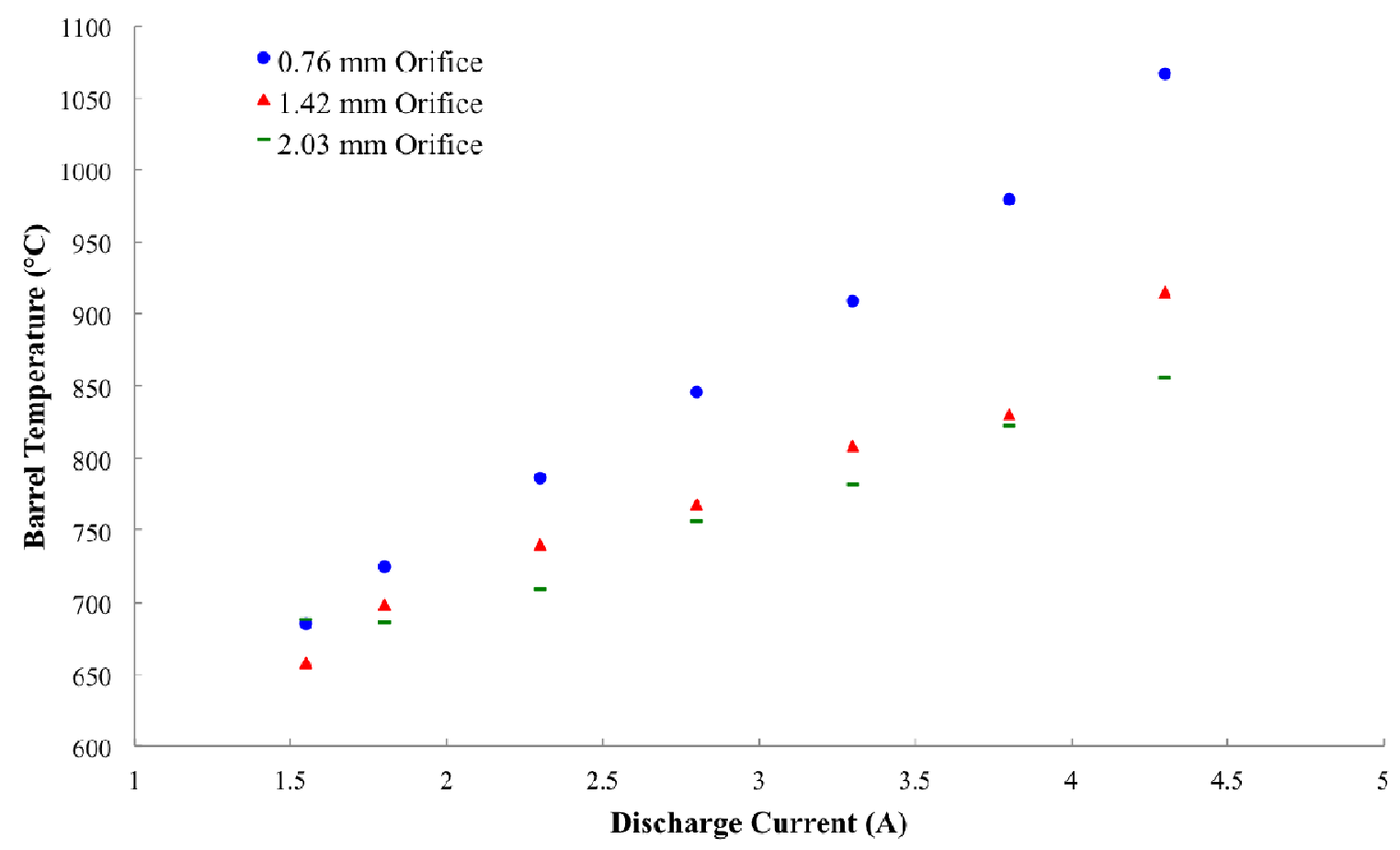

Figure 4.4: The measured barrel temperature of cathodes with three different orifice sizes as a function of the discharge current. The mass flow rate was fixed at $4 \mathrm{sccm}$.

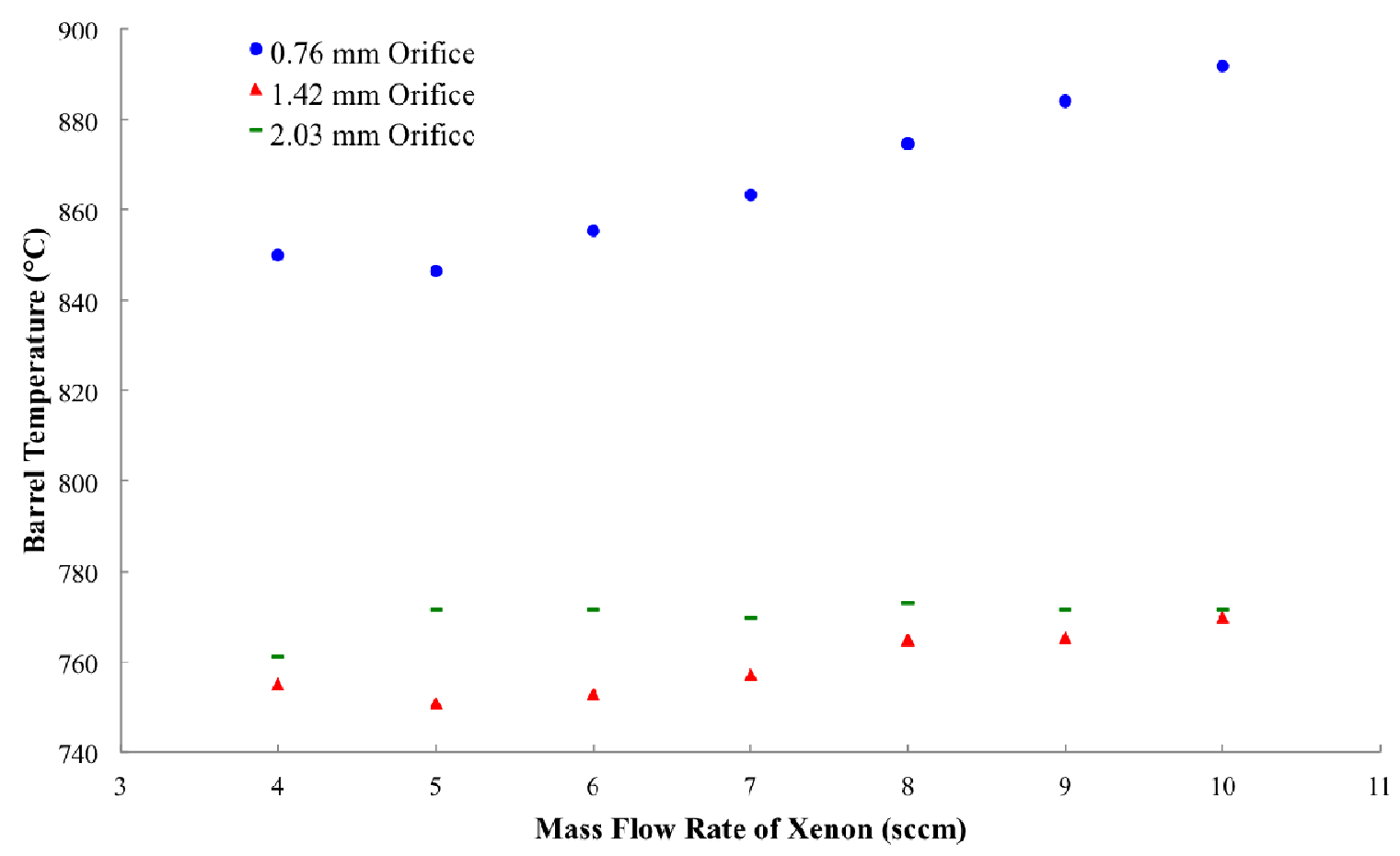

Figure 4.5: The measured barrel temperature of cathodes with three different orifice sizes as a function of mass flow rate. The discharge current was fixed at $2.8 \mathrm{~A}$. 


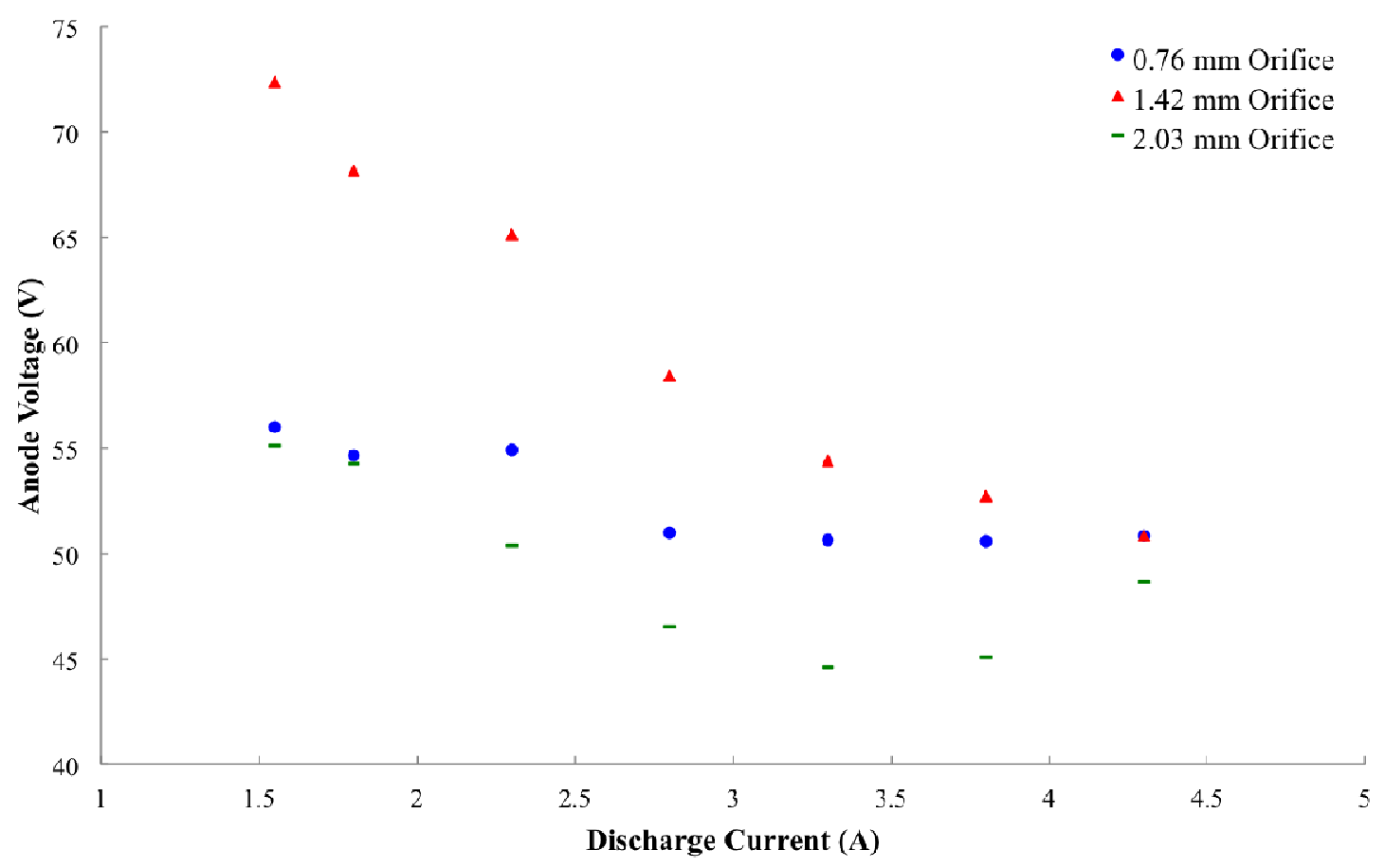

Figure 4.6: Anode voltage behavior with three different orifice sizes as a function of the discharge current. The mass flow rate was fixed at $4 \mathrm{sccm}$.

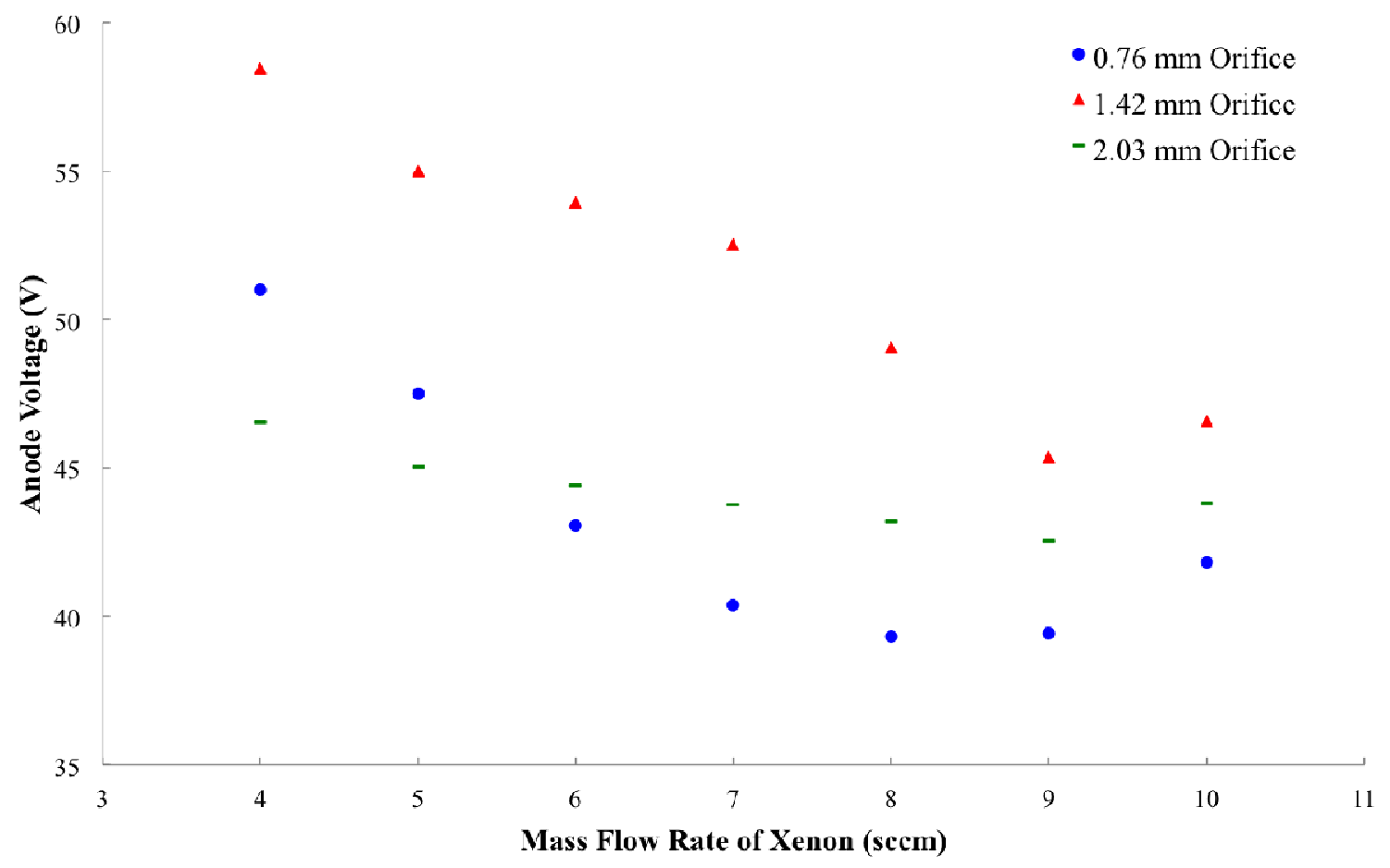

Figure 4.7: Anode voltage behavior with three different orifice sizes as a function of mass flow rate. The discharge current was fixed at $2.8 \mathrm{~A}$. 
decreases, the internal cathode pressure increases, which moved the peak plasma density closer to the orifice plate and the position of the thermocouple, which would be reflected in an increased temperature. At very low discharge currents, all three cathodes were observed to operate at similar low temperatures. It is likely that at this condition, either orifice heating was not the primary heating mechanism due to the low orifice plasma density (as a result of the low discharge current) in the $0.76 \mathrm{~mm}$ orificed cathode, or the peak plasma density was no longer high enough to heat the surface under the thermocouple significantly.

One would expect to see a decrease in anode voltage as the orifice size was increased. However, as shown in Figure 4.6 and Figure 4.7, the anode voltages observed with the $0.76 \mathrm{~mm}$ orifice and the $2.03 \mathrm{~mm}$ orifice were fairly similar, while the $1.42 \mathrm{~mm}$ orifice exhibited the highest anode voltages. However, the difference decreased as the plasma density increased, as evidenced by the convergence of all three data sets in both Figure 4.6 and Figure 4.7 with increasing discharge current and mass flow rate. It is possible that the cathode with the $1.42 \mathrm{~mm}$ diameter orifice was particularly susceptible to operating in a plasma mode characterized by high anode voltages. Alternatively, the anode may have not been adequately cleaned before testing the $1.42 \mathrm{~mm}$ orificed cathode, leading to the observed increased anode voltages.

\subsection{Iodine Testing}

Iodine has recently attracted interest as an alternative electric propulsion propellant [6-9]. Iodine has an atomic mass similar to xenon with slightly larger ionization cross-sections (for both $\mathrm{I}$ and $\mathrm{I}_{2}$ ). Additionally, it can be stored in low pressure tanks in the solid phase, eliminating the need for the large, high pressure storage solutions 
mandated by xenon [7]. The increased reactivity when compared to xenon was a concern due to the halogenic nature of iodine [8], especially when the susceptibility to contamination of Ba-W hollow cathodes was considered. Given the observed resistance to contamination of the electride hollow cathode, it appeared to be an attractive alternative cathode technology.

An electride hollow cathode fabricated according to the procedure described in Chapter 3 was delivered to Busek Co. Inc. to perform the iodine compatibility testing. Busek spent several years [9] developing an iodine propulsion program, which included a feed system. The feed system incorporated a heated iodine reservoir with a pressure transducer that could be used to quantify the approximate flow rate. All lines between the reservoir and the cathode were heated to prevent iodine condensation. The reservoir was weighed after each day of operation, allowing for the development of a flow rate calibration curve from the measured reservoir pressure. The electride hollow cathode testing was conducted in a 1.2-m diameter, 1-m long stainless steel diffusion-pumped chamber. The cathode was tested in a diode configuration with a ring anode as shown in Figure 4.8.

The cathode was successfully started on iodine from room temperature with no heater, following the start-up procedure described in Section 4.1. Almost 20 hours of operation with iodine was accumulated on a single C12A7 electride insert with no signs of electride degradation or contamination. The 20 -hour duration involved eight restarts from room temperature as well as an exposure to atmosphere, and no difficulty starting and operating the cathode was encountered.

The anode voltage as a function of discharge current was measured at a constant iodine flow rate of approximately $13 \mathrm{sccm}$. Data were recorded as the current was 


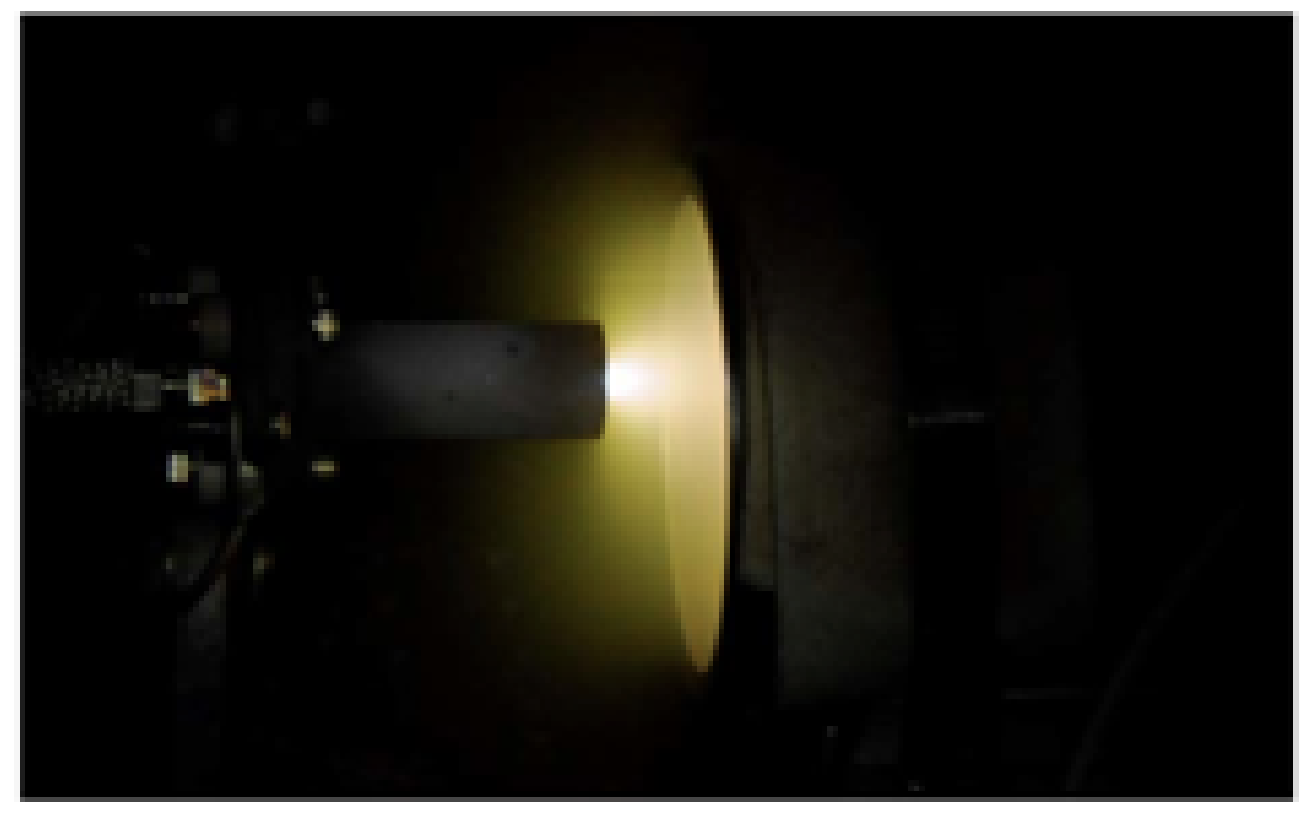

Figure 4.8: An electride hollow cathode in diode configuration operating on iodine. increased from $3 \mathrm{~A}$ to $15 \mathrm{~A}$, and as it was decreased from $15 \mathrm{~A}$ back down to $3 \mathrm{~A}$ over approximately one hour (Figure 4.9).

The cathode performance at lower iodine flow rates was also investigated, as shown in Figure 4.10. The discharge current was kept constant at $3 \mathrm{~A}$ with an additional $0.3 \mathrm{~A}$ collected by the keeper. The temperature of the iodine reservoir in the feed system was slowly decreased while the anode voltage was recorded. The anode voltage started to increase at flow rates below about $5 \mathrm{sccm}$. It is noted that flow rates shown in Figure 4.10 were calculated from the pressure transducer mounted on the iodine reservoir and from calibration data that were collected from iodine reservoir weight measurements made after each day of testing. It is not known what the internal pressure of the cathode was, but it is estimated to be approximately one Torr, and, consequently, there is a great deal of uncertainty regarding flow rate. We suspect that the flow rate at which the increase in 


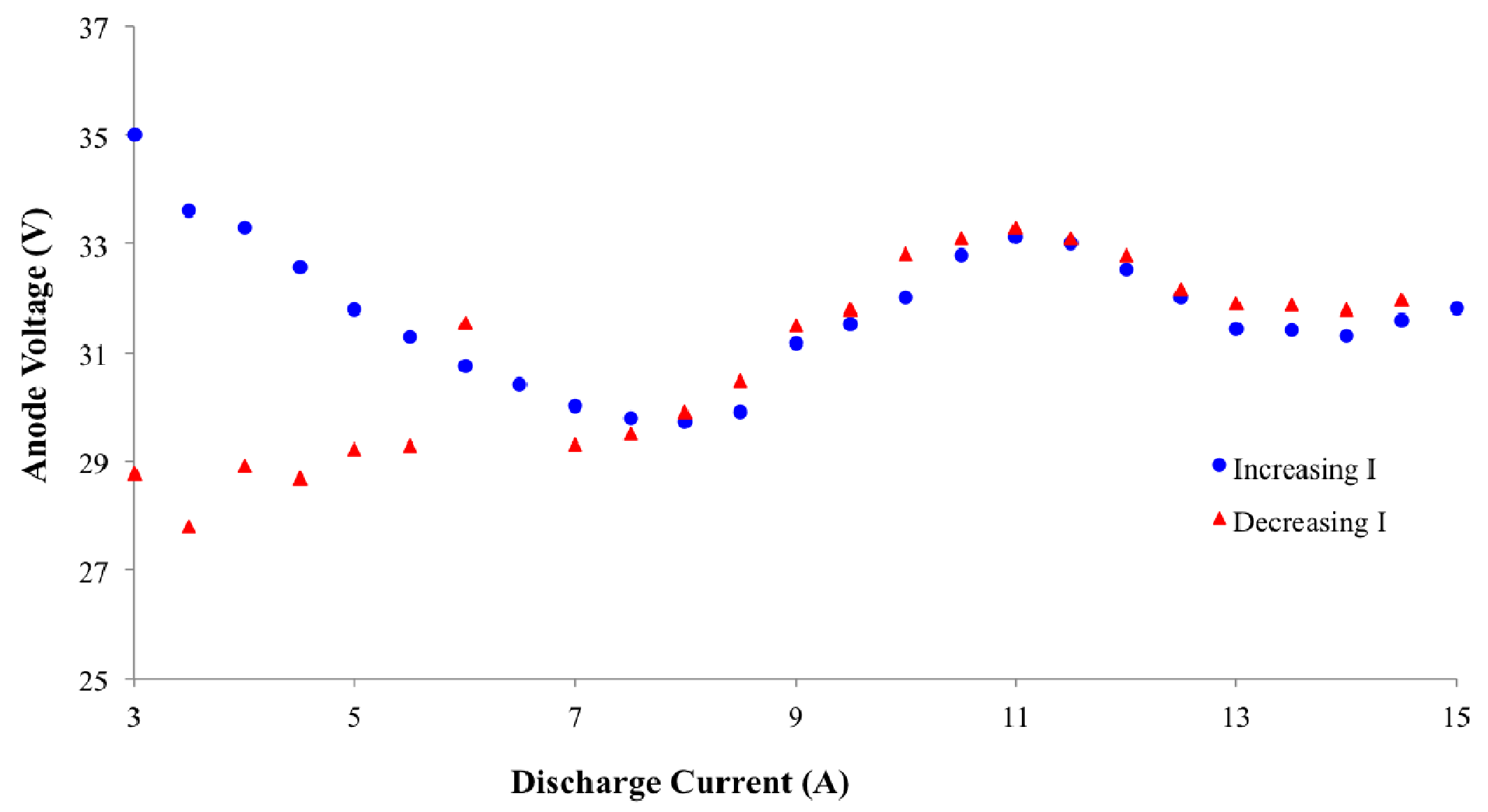

Figure 4.9: Anode voltage as a function of discharge current for an electride hollow cathode running on iodine with a constant flow rate of $13 \mathrm{sccm}$ and 0.3 A to the keeper. 
anode voltage was observed is lower than $5 \mathrm{sccm}$, because operation on xenon shows an increase in anode voltage at flow rates closer to $1 \mathrm{sccm}$ at a discharge current of $3 \mathrm{~A}$.

There was no evidence of any detrimental interaction between iodine and electride. No degradation in operation was observed over time, nor was there any increasing difficulty in starting the cathode. However, a black discoloration was observed on the outer surface of the tantalum cathode barrel and the stainless steel nut, and the tantalum radiation shielding was also discolored and damaged. The most likely explanation is that the iodine is reacting with the cathode structure materials to form iodine compounds. Tantalum will react with iodine to form tantalum pentaiodide (TaI5) starting at $300^{\circ} \mathrm{C}$ [10]. Iodine is known to react with stainless steel at temperatures as low as room temperature [11]. Using different refractory metals like tungsten or molybdenum for the barrel and radiation shielding material would most likely not prevent corrosion, as they react with iodine at elevated temperatures. The solution may be to use a graphite barrel with grafoil or platinum radiation shielding. Graphite will adsorb and desorb iodine with temperature fluctuations but will not corrode or react [12]. It would be possible to fabricate the cathode barrel and orifice plate from graphite and cover the downstream end of the orifice plate with a platinum plate. The platinum plate would prevent arcs from occurring between the graphite and the keeper during starting. Graphite erodes relatively quickly and malforms into peaks and tendrils when subjected to arcing, which is not desirable for long life applications. The platinum 
would help to alleviate this problem. Platinum will eventually corrode in the presence of iodine, although at a rate more than 150 times slower than that of tantalum [13]. 


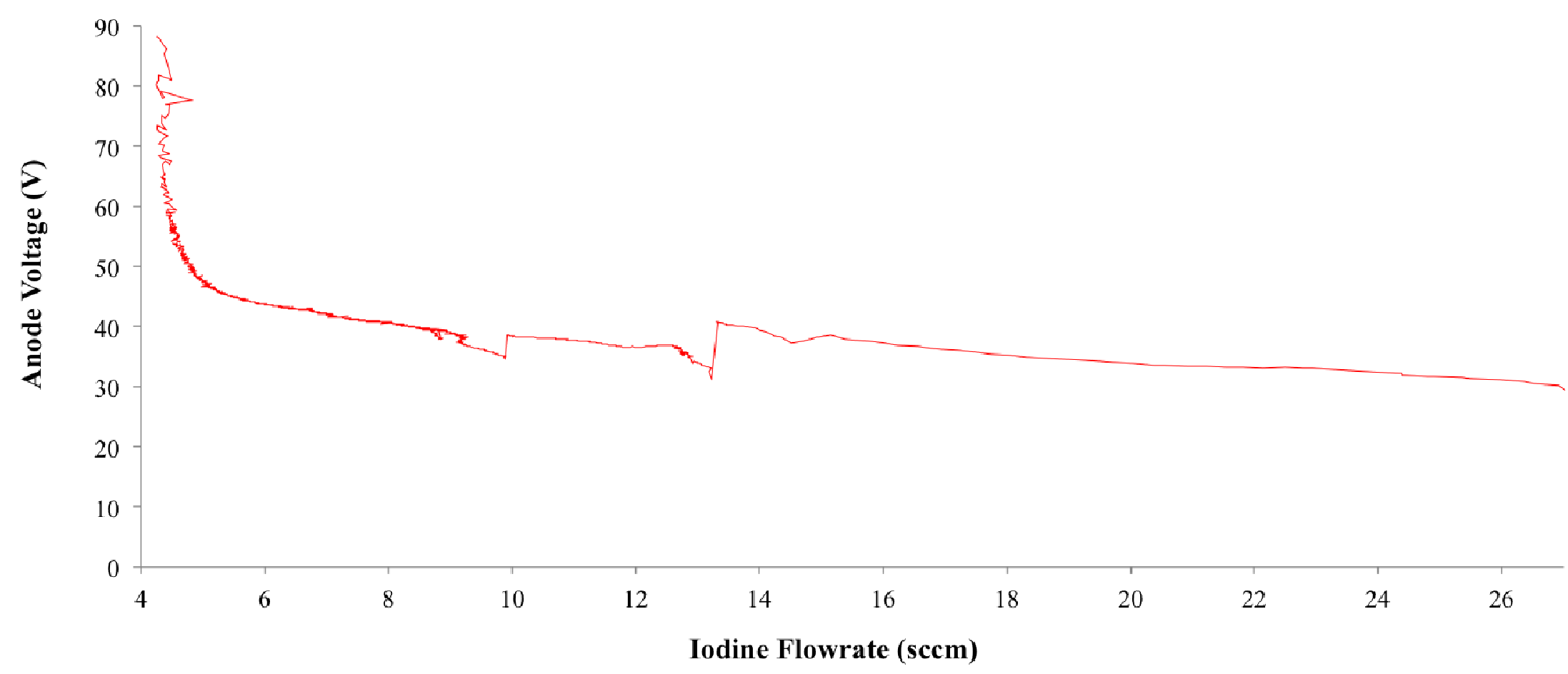

Figure 4.10: Anode voltage as a function of mass flow rate for an electride hollow cathode running on iodine. 


\section{References}

[1] L. Rand, J. Williams, and R. Hoyt, in 47th AIAA/ASME/SAE/ASEE Jt. Propuls. Conf. Exhib. (San Diego, California, 2011).

[2] D. L. Brown, Investigation of Low Discharge Voltage Hall Thruster Characteristics and Evaluation of Loss Mechanisms, University of Michigan, 2009.

[3] D. Goebel and I. Katz, Fundamentals of Electric Propulsion: Ion and Hall Thrusters (John Wiley \& Sons Inc, 2008).

[4] Y. P. Raizer, Gas Discharge Physics, 1st ed. (Springer, New York, NY, 1997).

[5] D. Goebel, R. Watkins, and K. Jameson, J. Propuls. Power 23, 552 (2007).

[6] J. Szabo, M. Robin, S. Paintal, B. Pote, and V. Hruby, in 48th AIAA/ASME/SAE/ASEE Jt. Propuls. Conf. (Atlanta, Georgia, 2012).

[7] J. Szabo, B. Pote, S. Paintal, M. Robin, A. Hillier, R. D. Branam, and R. E. Huffman, J. Propuls. Power 28, 848 (2012).

[8] J. Szabo and M. Robin, in 49th AIAA/ASME/SAE/ASEE Jt. Propuls. Conf. (American Institute of Aeronautics and Astronautics, San Jose, California, 2013).

[9] J. Szabo, M. Robin, S. Paintal, B. Pote, V. Hruby, and C. Freeman, in Proc. 33rd Int. Electr. Propuls. Conf. (Washington, DC, 2013).

[10] P. A. Schweitzer, Corrosion Engineering Handbook, 2nd ed. (Marcel Dekker, Inc., New York, NY, 1996).

[11] J. C. Wren, G. A. Glowa, and J. Merritt, J. Nucl. Mater. 265, (1999).

[12] K. Iwamoto and J. Oishf, J. Nucl. Sci. ... 5, (1968).

[13] G. Y. Lai, High Temperature Corrosion and Materials Applications (ASM International, Materials Park, OH, 2007). 


\section{Zero-Dimensional Phenomenological Model}

This chapter describes the development of a zero-dimensional model of the plasma internal to an electride hollow cathode. Work done previously in the area is discussed, and the changes implemented to create the current model are examined. The accuracy of the model is reviewed, and suggestions for future efforts are made.

\subsection{Previous Hollow Cathode Model Research}

In 1978, Ferreira and Delcroix [1] developed a comprehensive, self-consistent model of the interior plasma in a hollow cathode. Using an orifice-less hollow cathode in a diode configuration, the authors measured the wall temperature and internal pressure as a function of the gas flow rate and discharge current. They modeled the spatial variations of the internal plasma according to the radial diffusion equation, and found the absolute plasma density using a collisional model of the primary electrons. The collisional model was a function of the sheath potential, which allowed for the determination of the potential drop at the cathode wall. Ferreira and Delcroix were able to validate their approach through positive comparisons between the theoretical and experimental values of the cathode wall temperature and the internal pressure.

Ferreira and Delcroix's model was not able to explain the observed dependence of length of the internal plasma column on the gas flow rate and discharge current. Additionally, it only considered a hollow cathode with no orifice, which considerably simplified the simulation.

Building on the work done by Ferreira and Delcroix, Siegfried and Wilbur $[2,3]$ devised a zero-dimensional model of the internal plasma of an orificed hollow cathode 
operated on mercury. The underlying foundations of both models were very similar. A diffusion-based description of the plasma was used in conjunction with an energy balance applied at the insert surface to relate the plasma volume equation to the surface emission equations. Siegfried and Wilbur proved through theoretical and experimental results that the emission of electrons off of the insert surface was almost entirely due to a field-assisted thermionic emission mechanism, rather than through photoelectric emission or secondary electron emission phenomena.

Additionally, Wilbur and Siegfried used a segmented thermionic emitter in which the segments were isolated from one another to quantify the length of insert responsible for electron emission. They found that only the first several millimeters of the insert were responsible for the cathode emission, due to the ion production region (and thus the internal plasma column) being limited to several electron mean free path-lengths from the orifice plate. This limitation was thought to exist due to the ion formation resulting almost solely from primary electron collisions combined with the higher likelihood of finding primary electrons at the downstream end of the plasma column since that was where all the electrons were being directed. Wilbur and Siegfried validated this explanation by correlating the theoretical emission length with the emitted current distribution measured during the segmented insert experiment.

An updated zero-dimensional model for a hollow cathode was formulated by Katz et al [4-6]. Due to advances made during the past several decades in fast-moving probe measurement techniques, Katz et al were able to utilize experimentally-measured values for electron temperature, plasma potential, and internal plasma density into their model. This allowed a more accurate model to be developed that required considerably less 
guesswork than previous iterations. Also, because the cross sections related to xenon were extensively measured by Miller et al [7], Katz et al were able to leave out complicated cross section estimates.

As in the early models discussed, a control volume around the insert surface was utilized in conjunction with equations for the conservation of energy and current. Assuming steady-state conditions, the energy leaving the insert must be equal to the energy deposited into the insert from plasma bombardment processes. The heat loss was quantified with a thermal model [4] and the plasma properties were deduced through the conservation of energy and current. More specifically, the electron temperature was solved for using the cylindrical diffusion equation

$$
\left(\frac{R}{\lambda_{01}}\right)^{2} n_{o} \sigma_{i}\left(T_{e}\right) \sqrt{\frac{8 k T_{e}}{\pi m}}-D=0
$$

where $\boldsymbol{R}$ was the radius of the internal cathode volume, $\boldsymbol{m}$ was the mass of an electron, $\boldsymbol{n}_{\boldsymbol{o}}$ was the neutral density, $\sigma_{i}\left(T_{e}\right)$ was the xenon ionization cross section, and $\boldsymbol{D}$ was the diffusion coefficient found from the ion and electron mobilities. Once the electron temperature was known, the plasma density and sheath potential could be found by combining the conservation of energy applied to a control volume around the internal cathode plasma and the conservation of energy applied to a control volume around the insert surface. The energy balance applied to the internal cathode plasma was written

$$
I_{t} \phi_{s}+R I_{e}^{2}=I_{i} U^{+}+\frac{5}{2} T_{e V} I_{e}+\left(2 T_{e V}+\phi_{s}\right) I_{r} e^{-\phi_{s} / T_{e V}} .
$$

Equation (5.2) was structured so that that the control volume around the plasma was bounded prior to the sheath at the insert surface. The left side of (5.2) was the energy flowing into the plasma control volume, and the right side was the energy flowing out. $\boldsymbol{I}_{t} \boldsymbol{\Phi}_{\boldsymbol{s}}$ 
was the energy brought into the plasma by thermionically-emitted electrons that flowed from the internal cathode surface and were accelerated through the sheath potential and $\boldsymbol{R} \boldsymbol{I}_{e}{ }^{2}$ was the joule heating of the plasma as the discharge current was pulled through the control volume to the orifice. $\boldsymbol{I}_{+} \boldsymbol{U}^{+}$was the ionic potential energy removed from the plasma in the form of ionization potential and (5/2)T $T_{e V I} I_{e}$ was the enthalpy of the electrons leaving the control volume that flow to the orifice. The term

$$
I_{r} e^{\frac{-\phi_{s}}{T_{e V}}}\left(2 T_{e V}\right)
$$

was the kinetic energy of the electrons lost from the plasma to the internal cathode surface, in which $\boldsymbol{I}_{\boldsymbol{r}}$ was the random thermal current, and

$$
I_{r} e^{\frac{-\phi_{s}}{T_{e V}}}\left(\phi_{s}\right)
$$

accounted for the potential energy carried by these electrons. Both terms included an attenuation factor to account for the electrons that made it partially through the sheath but had inadequate amounts of energy and were returned to the plasma before reaching the cathode surface.

An energy balance was also applied to the control volume placed at the internal cathode surface

$$
H(T)+I_{t} \phi_{w f}=I_{i}\left(U^{+}+\phi_{S}+\frac{T_{e V}}{2}-\phi_{w f}\right)+\left(2 T_{e V}+\phi_{w f}\right) I_{r} e^{\frac{-\phi_{S}}{T_{e V}}} .
$$

The terms on the right side of the equation accounted for the energy leaving the cathode surface, where $\boldsymbol{H ( T )}$ was the total heat transfer from the internal cathode surface to the cathode structure and $\boldsymbol{I}_{t} \boldsymbol{\Phi}_{w f}$ was the thermionic emission cooling term describing the energy removed by the emitted electrons when they were boiled off the surface. The first 
grouping of terms on the right side of the equation accounted for the power deposited into the cathode surface by the ion bombardment current. $\boldsymbol{I}_{i} \boldsymbol{U}^{+}$was the ionization energy supplied by the ions when they hit the cathode surface, $\boldsymbol{I}_{i} \boldsymbol{\Phi}_{\boldsymbol{s}}$ was the energy gained by the ions when they fell through the plasma sheath en route to colliding with the cathode surface,

$$
I_{i} \frac{T_{e V}}{2}
$$

was the energy gained by the ions prior to arriving at the sheath boundary edge, also known as the pre-sheath or Bohm energy, and $-\boldsymbol{I}_{i} \boldsymbol{\phi}_{\boldsymbol{w} f}$ was the energy lost by the cathode surface when electrons in the surface conduction band jumped to the surface to neutralize ions on the surface.

The second grouping of terms on the right side of the equation was the power deposited into the cathode surface by the returning electron current. The rate of electrons that arrived at the surface was equal to the random thermal electron current multiplied by an attenuation term

$$
e^{\frac{-\phi_{S}}{T_{e V}}}
$$

The kinetic energy deposited by the electrons that made it to the cathode surface (assuming a Maxwellian velocity distribution) was

$$
I_{r} e^{\frac{-\phi_{s}}{T_{e V}}}\left(2 T_{e V}\right)
$$

and the energy given up by the electrons to the surface when they transitioned from the vacuum level to the conduction band was 


$$
I_{r} e^{\frac{-\phi_{s}}{T_{e V}}}\left(\phi_{w f}\right)
$$

The Katz et al model was adapted to construct a model of the internal plasma in an electride hollow cathode.

\subsection{Zero-Dimensional Electride Hollow Cathode Model}

A zero-dimensional model of the plasma inside an electride hollow cathode was desired to gain insight into the operation of a cathode with a low work function, nonradially symmetric emission surface. The model was designed to answer two questions:

1. How do the interior plasma conditions of a C12A7 electride hollow cathode compare with those of a traditional hollow cathode?

2. How does the C12A7 electride emission surface temperature compare with the operating temperature of the cathode?

There are several challenges in the application of a zero-dimensional model to an electride hollow cathode. One such challenge is that in the cathodes described by the models discussed previously, the primary surface exposed to plasma bombardment was the low work function insert. In an electride cathode, most of the plasma-bombarded surface area is the graphite liner or the tungsten orifice plate, which have comparatively high work functions. Additionally, the geometries of the cathodes previously modeled were radially symmetric. The sliver-insert of a C12A7 electride cathode leads to radial non-symmetry, which adds complexity. To address these challenges, a two-step approach was utilized in the development of a C12A7 electride hollow cathode 0-D model. The first step involved a control surface around the graphite tube that accounted for the majority of the inner surface area. With this approach, the internal plasma properties could be 
calculated from a measured operating temperature, which helped to answer question \#1. In the second phase, those plasma properties were used to determine the energy deposited in the electride from plasma bombardment. This allowed for an estimation of the electride surface temperature to be made, answering question \#2. The remainder of Section 5.3 consists of a detailed walk-through of the application of the developed model to the case of an electride hollow cathode with a $1.42 \mathrm{~mm}$ diameter orifice operating on $4 \mathrm{sccm}$ of xenon with a discharge current of 3.3 A. Additional results are presented in Section 5.3.

Table 5.1: Geometry used to model the electride hollow cathode with a $1.42 \mathrm{~mm}$ diameter orifice.

\begin{tabular}{|l|c|c|}
\hline Inner radius of tantalum barrel & $\boldsymbol{r}_{\boldsymbol{i} \text {, } \text {, } a r r e l}$ & $2.67 \mathrm{~mm}$ \\
\hline Outer radius or tantalum barrel & $\boldsymbol{r}_{\boldsymbol{o} \text {, } \text {, arrel }}$ & $3.18 \mathrm{~mm}$ \\
\hline Inner radius of graphite insert & $\boldsymbol{r}_{\boldsymbol{i} \text {, graphite }}$ & $1.27 \mathrm{~mm}$ \\
\hline Outer radius of graphite insert & $\boldsymbol{r}_{\boldsymbol{o} \text {, graphite }}$ & $2.54 \mathrm{~mm}$ \\
\hline Orifice diameter & $\boldsymbol{d}_{\text {orifice }}$ & $1.42 \mathrm{~mm}$ \\
\hline Orifice length & $\boldsymbol{I}_{\text {orifice }}$ & $1.37 \mathrm{~mm}$ \\
\hline
\end{tabular}

\section{A. Determination of Neutral Pressure}

An operating temperature of $808{ }^{\circ} \mathrm{C}$ was measured experimentally using a thermocouple as described in Chapter 3. The neutral gas temperature in the cathode is assumed to be three times the wall temperature, $3242 \mathrm{~K}$ in this case. To calculate the internal cathode pressure, the viscosity of the xenon, $\zeta$, is found using the equation from the Katz et al model [6]

$$
\zeta=2.3 \times 10^{-4} T_{r}^{\left(0.71+\frac{0.29}{T_{r}}\right)}
$$


in which $\boldsymbol{T}_{\boldsymbol{r}}$ is the gas temperature divided by 289.7. To find the average pressure inside the cathode, the pressure directly upstream of the orifice, $\boldsymbol{P}_{2}$, is needed. The pressure at the downstream end of the orifice was assumed to be small in comparison, and as such, $\boldsymbol{P}_{\mathbf{2}}$ can be written as

$$
P_{2}=\sqrt{\frac{0.78 Q \zeta T_{r} l_{\text {orifice }}}{d_{\text {orifice }}^{4}}}
$$

where $\boldsymbol{Q}$ is the mass flow rate in sccm. For the currently discussed conditions, $\boldsymbol{P}_{2}$ is calculated to be 3.99 Torr. The pressure at the upstream end of the plasma column, $\boldsymbol{P}_{1}$, can now be found. The emission length, $\boldsymbol{L}_{\boldsymbol{e}}$, is assumed to be $3 \mathrm{~mm}$

$$
P_{1}=\sqrt{P_{2}+\frac{0.78 Q \zeta T_{r} L_{e}}{\left(2 r_{i, \text { graphite }}\right)^{4}}} .
$$

$\boldsymbol{P}_{1}$ is calculated to be 4.00 Torr, which gives an average pressure over the length of the plasma column of 4.00 Torr. The neutral density, $\mathrm{n}_{\mathrm{o}}$, is calculated with

$$
n_{o}=9.65 \times 10^{24} \frac{P_{a v g}}{T}
$$

and is found to be $1.19 \times 10^{22} \mathrm{~m}^{-3}$.

\section{B. Determination of Electron Temperature}

Katz et al [6] find the electron temperature, $\boldsymbol{T}_{\boldsymbol{e}}$, using the radial diffusion equation

$$
\left(\frac{r_{i, \text { graphite }}}{\lambda_{01}}\right) n_{o} \sigma_{i}\left(T_{e}\right) \sqrt{\frac{8 e T_{e V}}{\pi m}}-D=0
$$

where $\lambda_{01}$ is the first zero of the zero-order Bessel function 2.405 , and $\boldsymbol{D}$ is a diffusion coefficient written as 


$$
D=\frac{e}{M} \frac{\left(T_{i V}+T_{e V}\right)}{\sigma_{C E X} n_{o} v_{\text {scat }}}
$$

In equation (5.9), $\boldsymbol{M}$ is the mass of xenon, $\boldsymbol{T}_{\boldsymbol{i V}}$ is the ion temperature, $\boldsymbol{\sigma}_{C E X}$ is the charge exchange cross section, and $\boldsymbol{v}_{\text {scat }}$ is the ion thermal velocity, written as

$$
v_{\text {scat }}=\sqrt{\frac{e T_{i V}}{M}} .
$$

The ionization cross section, $\boldsymbol{\sigma}_{i}\left(\boldsymbol{T}_{\boldsymbol{e}}\right)$ averaged over a Maxwellian temperature distribution around $\boldsymbol{T}_{\boldsymbol{e}}$, is calculated by finding the area under the curve

$$
y(E)=f(E) Q(E)
$$

where $\boldsymbol{f}(\boldsymbol{E})$ is the Maxwellian energy distribution function (5.12) and $\boldsymbol{Q}(\boldsymbol{E})$ is the ionization cross section as a function of electron temperature for xenon as reported in Rapp and Englander [8]

$$
f(E)=2 e \sqrt{\frac{e E}{\pi\left(e T_{e V}\right)^{3}}} e^{-\frac{E}{T_{e V}}} .
$$

Because $\boldsymbol{D}$ and $\boldsymbol{\sigma}_{\boldsymbol{i}}$ are both functions of $\boldsymbol{T}_{\boldsymbol{e}}$, equation (5.8) must be iteratively solved in order to obtain an electron temperature. In the electride hollow cathode model, a guess is first made for $\boldsymbol{T}_{\boldsymbol{e V}}$. The ionization cross section for a Maxwellian distribution of electrons centered around $\boldsymbol{T}_{\boldsymbol{e V}}$ is calculated. The ion temperature is assumed to be equal to the neutral gas temperature of $3243 \mathrm{~K}$, which corresponds to an ion thermal velocity of 453.13 $\mathrm{m} / \mathrm{s}$. The charge exchange cross section is assumed to be $1 \times 10^{-18} \mathrm{~m}^{-2}$. With these values, the ion thermal velocity can be found, which leads to the calculation of the diffusion coefficient, which allows for the solving of (5.8) for the electron temperature. After the 
iterative process, the electron temperature was found to be $2.27 \mathrm{eV}$, which leads to an ionization cross section of $1.04 \times 10^{-22} \mathrm{~m}^{-2}$ and a diffusion coefficient of $0.348 \mathrm{~m}^{2} / \mathrm{s}$.

\section{Heat Transfer Model}

To find the plasma density and sheath potential inside the cathode, a heat transfer model is needed to supply one side of the energy balance. The energy balance is based on the assumption that the cathode is operating at steady-state. As such, the heat leaving the cathode thermally must be equal to the power deposited in the cathode from the plasma. The basis of the heat transfer model utilized is the assumption that the temperature of the graphite insert along the length of the plasma column is equal to the temperature measured by the thermocouple on the outside of the barrel. The validity of this simplification is supported by Salhi et al [9], who showed that the temperature distribution on the exterior surface of the cathode barrel was within approximately ten degrees of the insert surface temperature distribution. The heat transfer model used for the electride hollow cathode is illustrated in Figure 5.1.

The conduction away from the control volume, $\boldsymbol{q}_{\text {cond, }}$, is written as

$$
q_{\text {cond }}=\frac{\left(T_{S}-T_{B}\right) k A}{L}
$$

where $\boldsymbol{T}_{\boldsymbol{s}}$ is the temperature measured at the surface by the thermocouple, and $\boldsymbol{T}_{\boldsymbol{B}}$ is the temperature of the cathode base, assumed to be $150{ }^{\circ} \mathrm{C} . \quad \boldsymbol{k}$ is the thermal conductivity of tantalum, $\boldsymbol{A}$ is the cross-sectional area of the tantalum barrel, and $\boldsymbol{L}$ is the length of the cathode barrel minus the length of the control volume, which in this case is assumed to be $\boldsymbol{L}_{\boldsymbol{e}}(\boldsymbol{L}$ is $16 \mathrm{~mm})$. For the measured operating temperature of $808{ }^{\circ} \mathrm{C}, \boldsymbol{q}_{\text {cond }}$ is found to be $9.85 \mathrm{~W}$. 


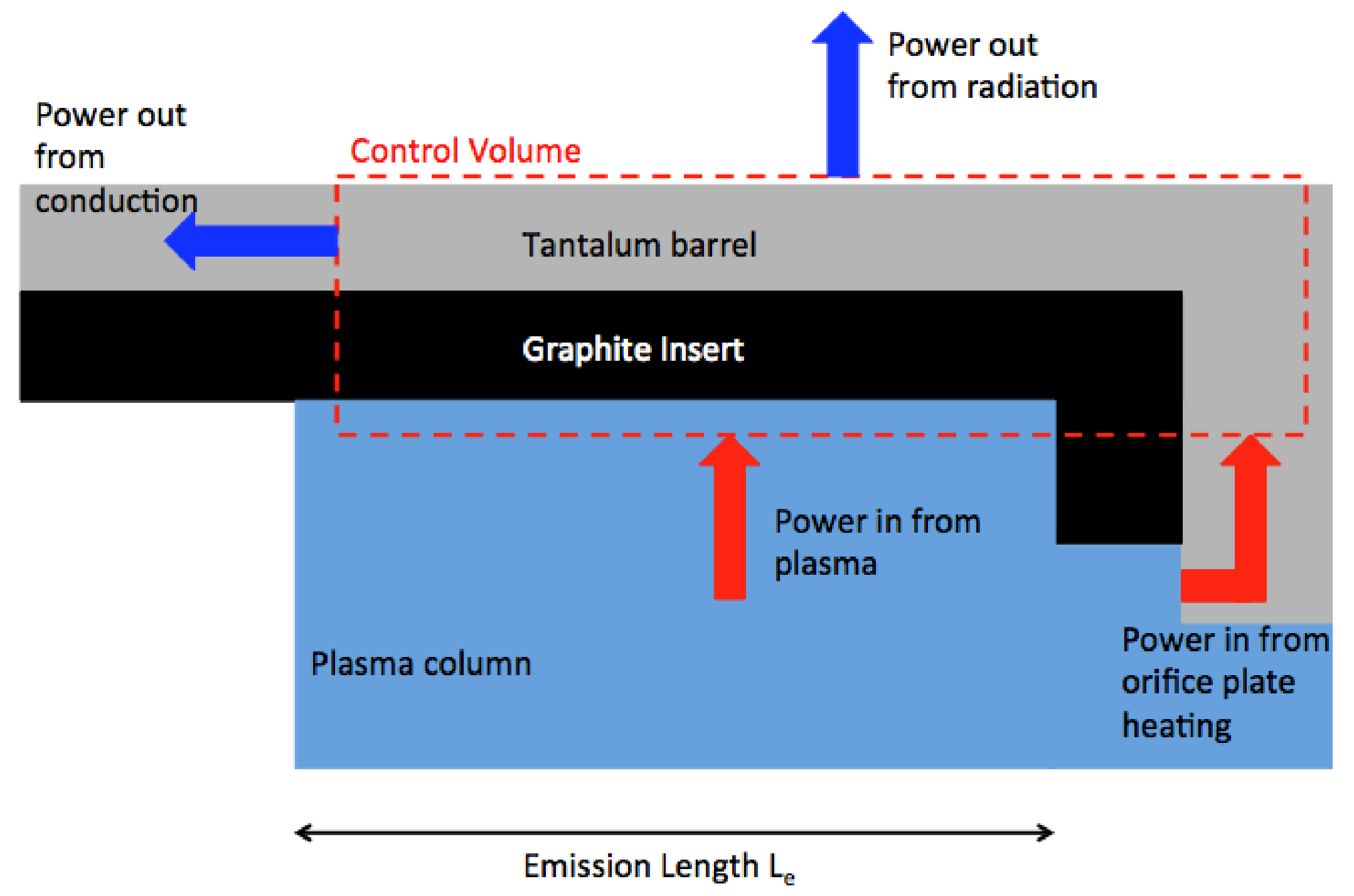

Figure 5.1: Schematic of the energy balance applied to the control volume drawn at the insert surface. 
The radiation away from the control volume, $\boldsymbol{q}_{\mathbf{r a d}}$, is written as

$$
q_{\text {rad }}=\varepsilon \sigma A\left(T_{s}^{4}-T_{\text {surr }}^{4}\right)
$$

where $\varepsilon$ is the emissivity of tantalum, $\sigma$ is the Stefan-Boltzmann constant, $\boldsymbol{T}_{\text {surr }}$ is the temperature of the surroundings and is specified to be room temperature, and $\boldsymbol{A}$ is the cathode perimeter multiplied by $\boldsymbol{L}_{\boldsymbol{e}}$. It was assumed that there was no radiation through the ten layers of tantalum foil radiation shielding, so $\boldsymbol{A}$ only accounts for the exposed area of the barrel, which in this case is $3.19 \times 10^{-4} \mathrm{~m}^{2}$. The temperature used in (5.14) for $\boldsymbol{T}_{s}$ is $752 \mathrm{~K}$, which is an average of the measured thermocouple temperature and the base temperature. A more accurate method of calculating the radiation would be to divide the exposed barrel into multiple segments and specify an average temperature for each of them. However, even if the temperature of the exposed barrel is set equal to the measured thermocouple temperature, which is presumed to be measuring the hottest point of the barrel, the resulting radiative heat loss changes only by $1.13 \mathrm{~W}$, which is not enough of a difference to vary considerably the resulting plasma properties. For the electride hollow cathode with a $1.42 \mathrm{~mm}$ diameter orifice, the radiation from the control volume to its surroundings is calculated to be $1.57 \mathrm{~W}$.

Orifice plate heating is the power deposited on the interior surface of the thoriated tungsten orifice plate by plasma bombardment. The decision to consider orifice plate heating and not orifice heating was based partially on results from more thorough twodimensional cathode models [5] and partially on the operating temperatures measured during the operation of electride hollow cathodes with different orifice sizes. As shown in Chapter 4 , there did not appear to be a significant difference in the operating temperature of a $1.42 \mathrm{~mm}$ diameter orifice when compared to a $2.03 \mathrm{~mm}$ orifice. As discussed by Katz et 
al [5], for cathodes with a orifice diameter to orifice length ratio greater than one, orifice plate heating becomes the dominant plasma heating mechanism over heating within the barrel of the orifice. The orifice diameter to length aspect ratio of the $1.42 \mathrm{~mm}$ electride hollow cathode is 1.04 .

The total heat flow out of the control volume is calculated as the heat lost through conduction and radiation minus the heat in from the orifice plate heating. Because the power deposited in the form of orifice plate heating is dependent on the internal plasma properties, the heat transfer model is iterative by necessity.

\section{Calculation of Sheath Potential and Plasma Density}

Figure 5.2 illustrates the two control volumes that are used to model the internal cathode plasma. Control volume \#1 is placed around the insert surface and is defined by (5.3). Now that the thermal heat loss is known, the power input from plasma bombardment is also known. Control volume \#2 is placed around the plasma column and is defined by equation (5.2). Because the power leaving control volume \#2 through plasma bombardment is known, the power into the plasma column generated by the plasma is also known.

To simplify the expression for sheath potential, an assumption is made that half the electron temperature is much less than the ionic potential plus the sheath potential. Given a calculated electron temperature of $2.27 \mathrm{eV}$, an ionization potential of $12.3 \mathrm{eV}$, and a predicted sheath potential on the order of $10 \mathrm{~V}$, this is a good assumption. Thus, the sheath potential can be simplified, as in the Katz et al model [6], as 


$$
\phi_{s}=\frac{H(T)}{I_{e}}+\frac{5}{2} T_{e V}+\phi_{w f}-I_{e} R
$$

A work function of $4.62 \mathrm{eV}$, the literature value for graphite [10], is used for $\boldsymbol{\phi}_{w f}$. The discharge current, $\boldsymbol{I}_{\boldsymbol{e}}$, is $3.3 \mathrm{~A}$ as specified previously. The plasma resistance, $\boldsymbol{R}$, is calculated by Katz et al [6] as

$$
R=\frac{1}{\varepsilon_{o} \tau_{e} \omega_{p}^{2}} \frac{l}{\pi r_{i, \text { graphite }}}
$$

where $\boldsymbol{\varepsilon}_{\boldsymbol{o}}$ is the permittivity of free space. $\boldsymbol{l}$ is the average conduction path length, and is estimated to be the distance from halfway along the emission length to the center of the cathode at the upstream orifice entrance. For the electride hollow cathode, it is calculated to be $1.97 \mathrm{~mm} . \boldsymbol{\tau}_{e}$ is the mean time between electron-ion and electron-neutral collisions, and is calculated as

$$
\tau_{e}=\frac{1}{2.9 \times 10^{-12} \frac{n_{e} \ln \Lambda}{T_{e V}^{3 / 2}}+\sigma_{e n}\left(T_{e V}\right) n_{o} \sqrt{\frac{8 e T_{e V}}{\pi m}}}
$$

where $\sigma_{e n}\left(T_{e V}\right)$ is the electron-neutral cross section and is found using

$$
\sigma_{e n}\left(T_{e V}\right)=6.6 \times 10^{-19}\left(\frac{\frac{T_{e V}}{4}-0.1}{1+\left(\frac{T_{e V}}{4}\right)^{1.6}}\right) .
$$

The Coulomb logarithm $\operatorname{In} \boldsymbol{\Lambda}$ is calculated as

$$
\ln \Lambda=23-\frac{1}{2} \ln \left(\frac{1 \times 10^{-6} n_{e}}{T_{e V}^{3}}\right) .
$$




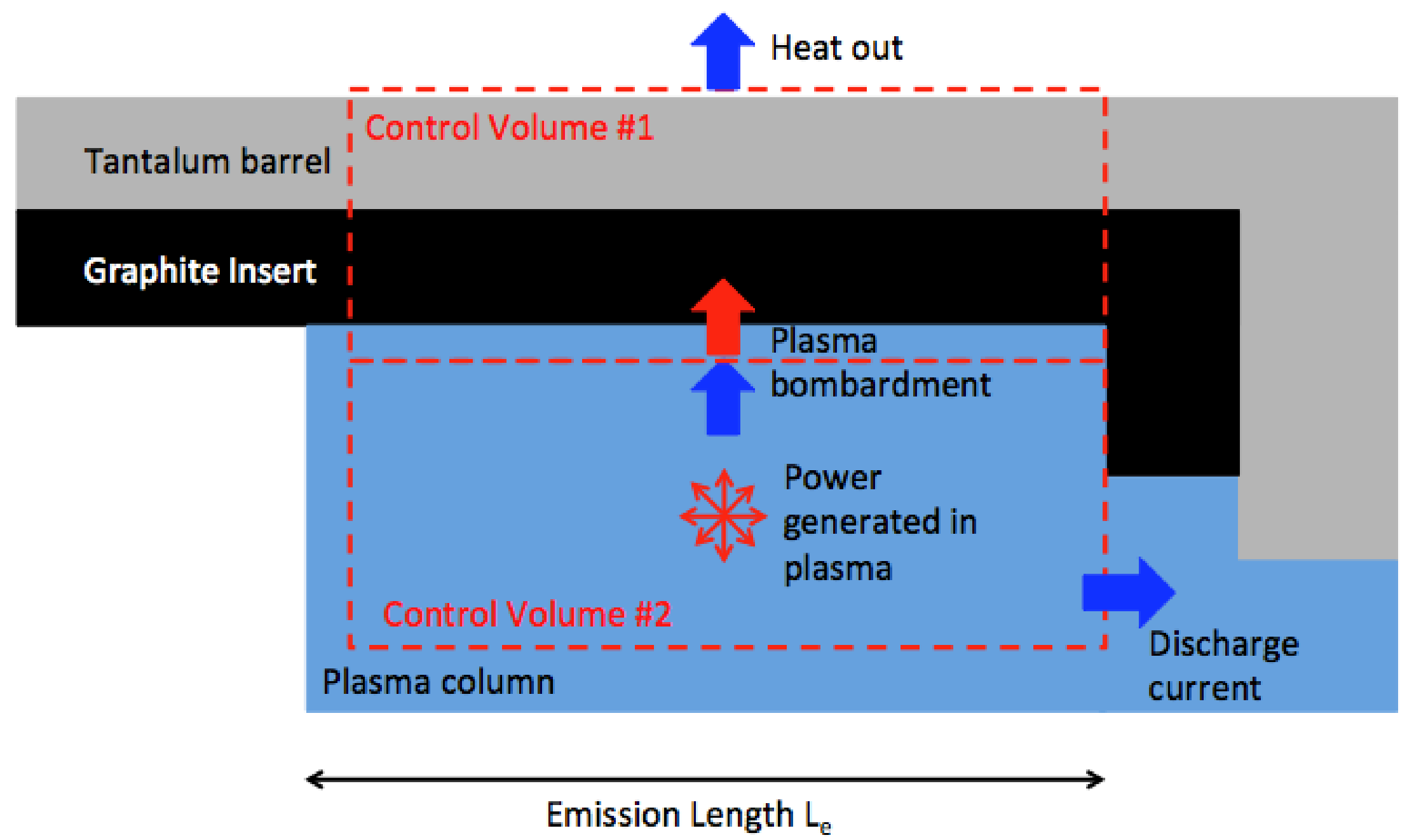

Figure 5.2: A schematic of the energy balance applied to two control volumes, one drawn around the graphite insert surface and the other around the internal cathode plasma column. 
The plasma density is solved for using the solution in the Katz et al model [6]

$$
n_{e}=\frac{R I_{e}^{2}-\left(\frac{5}{2} T_{e V}-\phi_{s}\right) I_{e}}{\left(f_{n} T_{e V} \sqrt{\frac{e T_{e V}}{2 \pi m}} e A e^{\frac{-\phi_{s}}{T_{e V}}}+n_{o} e\left\langle\sigma_{i} v_{e}\right\rangle V\left(U^{+}+\phi_{s}\right)\right)} .
$$

In (5.20), $\left\langle\sigma_{i} v_{e}\right\rangle$ is the reaction rate coefficient for ionizing collisions and is found from the area under the curve specified as

$$
g(E)=f(E) Q(E) \sqrt{\frac{2 e T_{e V}}{m}},
$$

and $\boldsymbol{f}_{\boldsymbol{n}}$ is the ratio of the average plasma density over the plasma column $\boldsymbol{n}_{\boldsymbol{e}}$ to the plasma density at the sheath edge. This is a parameter that must be experimentally-measured, and is unfortunately unknown for an electride hollow cathode. An estimate for $\boldsymbol{f}_{\boldsymbol{n}}$ of 0.05 has been used in the current model. That number is based on the measured plasma density ratio inside the NSTAR cathode [6], which is of similar size. Although there are significant differences between the NSTAR cathode and the electride cathode, the calculated plasma density and sheath potential are not highly sensitive to $\boldsymbol{f}_{\boldsymbol{n}}$. A change in $\boldsymbol{f}_{\boldsymbol{n}}$ from 0.05 to 0.5 results in a change in the calculated plasma density of only $0.6 \%$ and no detectable change in the sheath potential.

Because the Coulomb logarithm and collision time are dependent on the plasma density, the sheath potential must be solved for in an iterative fashion until a selfconsistent solution for the plasma density is found. For an electride hollow cathode with a $1.42 \mathrm{~mm}$ diameter orifice, $\omega_{\mathrm{p}}$ is found to be $6.55 \times 10^{11} \mathrm{~s}^{-1}$, the plasma resistance is $0.362 \Omega$, the sheath potential is $11.6 \mathrm{~V}$, the plasma density is $1.35 \times 10^{20} \mathrm{~m}^{-3}$, and orifice plate heating is $3.34 \mathrm{~W}$. 


\section{E. Determination of Electride Temperature}

The second step of the electride hollow cathode model utilizes a third control volume drawn around the electride surface, as shown in Figure 5.3. Once again, steadystate conditions are assumed, and an energy balance is used to calculate the electride temperature. Because the plasma conditions are known, the power flowing from the electride to the plasma is known. A thermal model is constructed, and the electride temperature is varied until the thermal model predicts a power flux equal and opposite to that from the plasma.

The thermal model assumes that the temperature of the electride is uniform over the entire length of the sliver, and that the only methods of heat flow are radiation from the electride to its surroundings and conduction. For the radiative heat transfer, it is assumed that the entire area (not just the emitting area in contact with the plasma) of the electride sliver is emitting to a surrounding area that is at the temperature of the experimentallymeasured cathode barrel. For the conductive heat transfer, it is assumed that the electride has a thermal conductivity of $2 \mathrm{~W} / \mathrm{mK}$, and that the electride sliver is in perfect thermal contact with the graphite tube.

To calculate the power into the electride from the plasma, the plasma density at the electride surface must be known. As discussed previously, the ratio of the average plasma density to the edge plasma density, $\boldsymbol{f}_{\boldsymbol{n}}$, can only be obtained via experimental measurement. As such, an educated guess must be used in the electride cathode model. For such a guess to be made, a closer examination of the plasma sheath structure at the edge of the electride where it meets the graphite is warranted. Katz et al [6] relate the ratio $f_{n}$ to the sheath potential using 


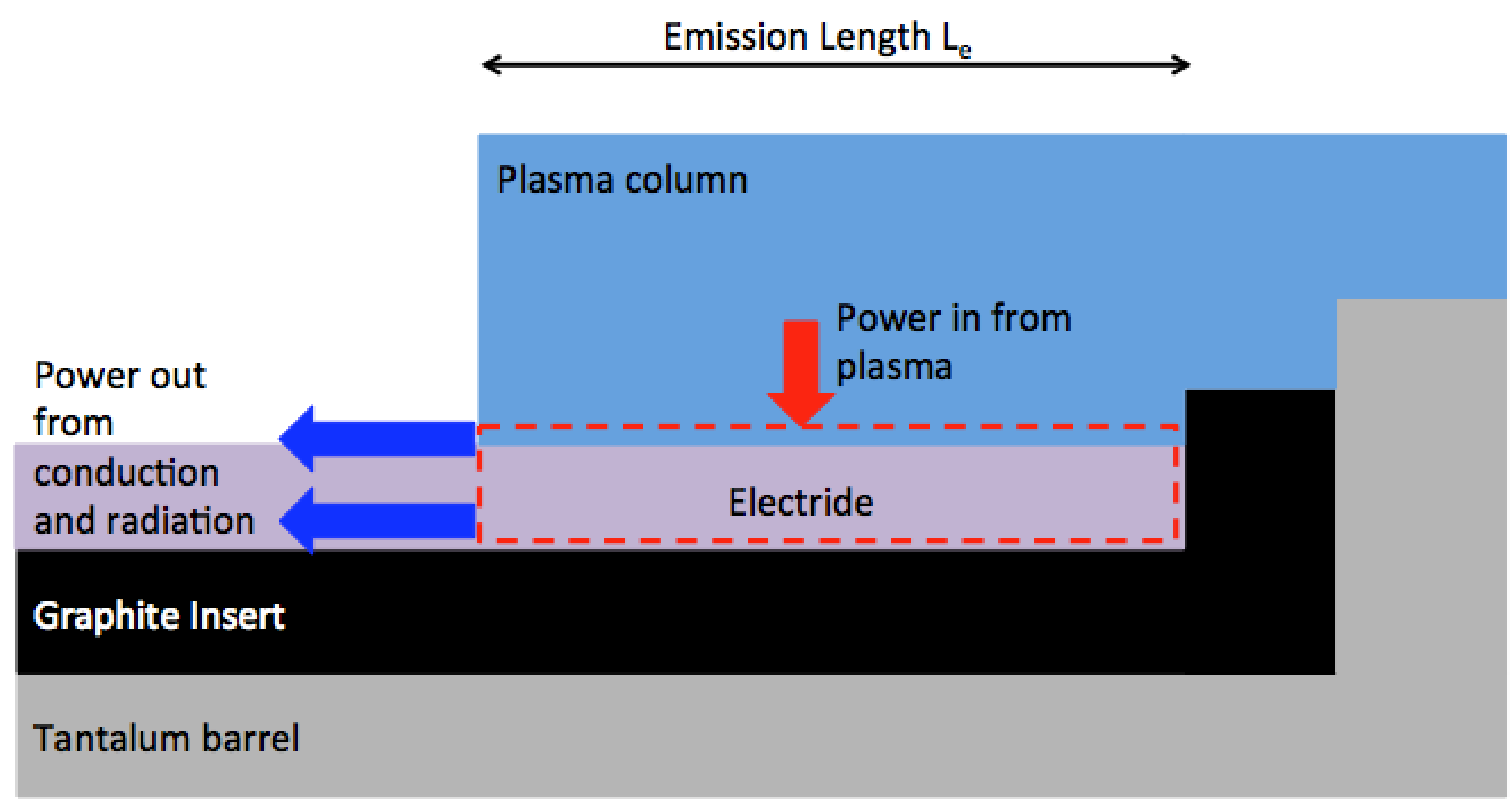

Figure 5.3: Schematic of the energy balance applied to the control volume drawn at the electride surface. 


$$
f_{n}=e^{\frac{-\left(\phi_{a x i s}-\phi_{s}\right)}{T_{e V}}}
$$

where $\boldsymbol{\phi}_{\text {axis }}$ is the plasma potential on the centerline of the cathode. Over the graphite, as calculated in Section 5.2, the sheath potential for an electride hollow cathode with a 1.42 $\mathrm{mm}$ diameter orifice was found to be $11.6 \mathrm{~V}$. The sheath is illustrated in Figure 5.4.

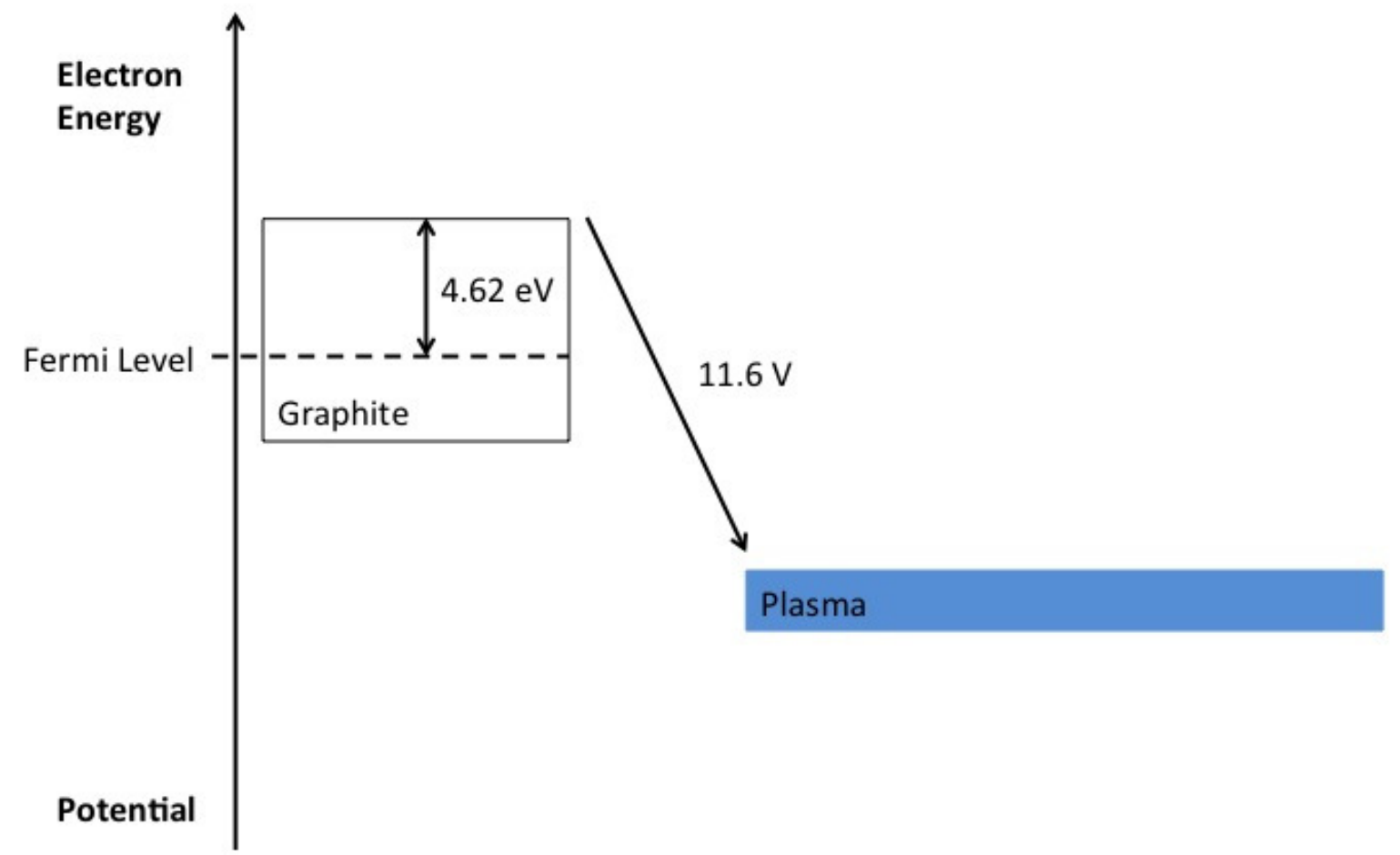

Figure 5.4: An illustration of the plasma sheath formed between the graphite surface and the internal cathode plasma.

When two conductive materials are in electrical contact, their Fermi levels align [11]. As such, if the work functions of the two materials are different, their surface energy states are at different potentials. This phenomenon becomes relevant when the plasma sheath at the boundary between the electride and the graphite is considered. Because the work function 
of graphite is $4.62 \mathrm{eV}$ and the work function of the electride may only be $0.76 \mathrm{eV}$, as discussed in Chapter 2, the electride surface is at a potential $3.86 \mathrm{~V}$ closer to the plasma than the graphite, as illustrated in Figure 5.5.

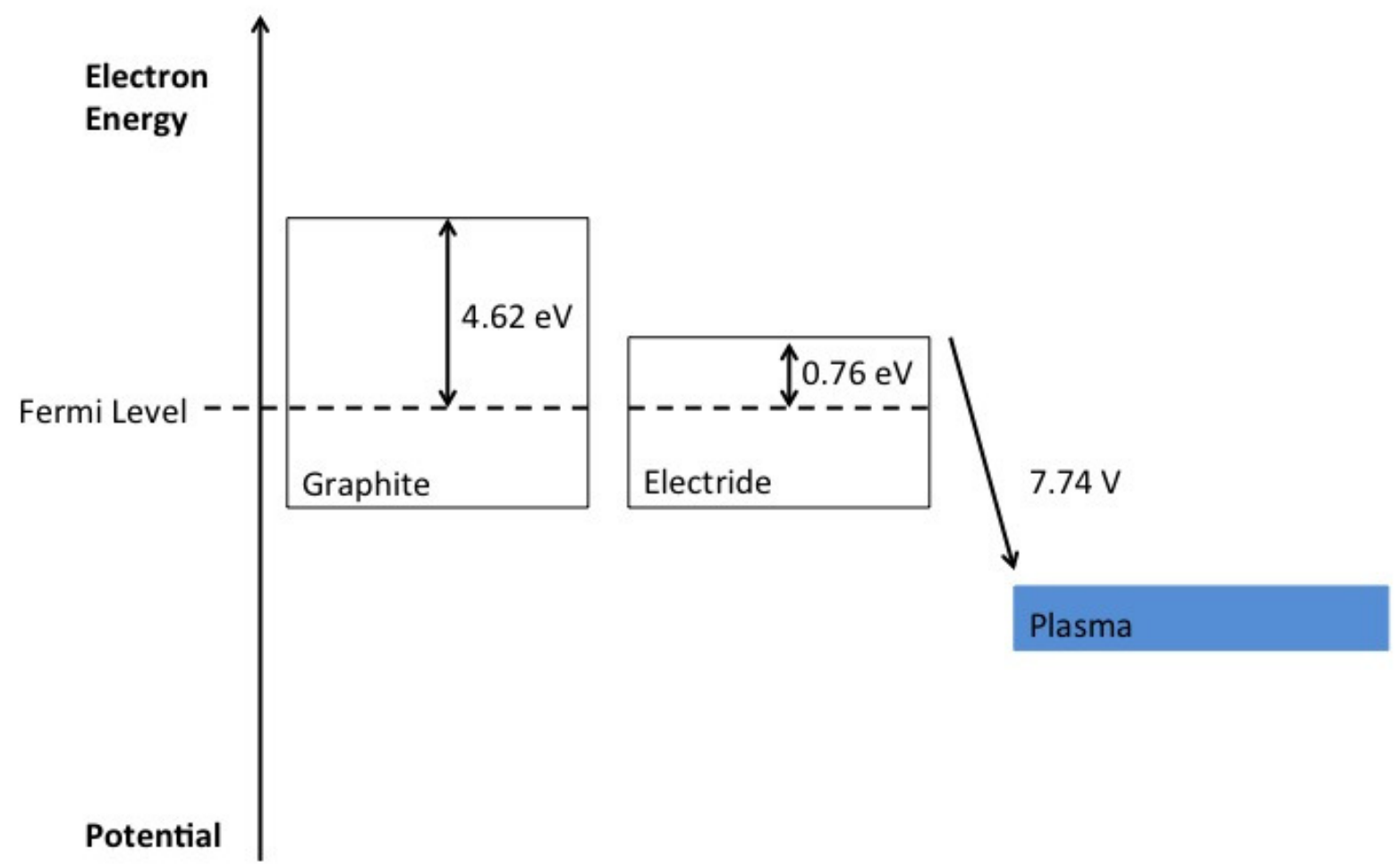

Figure 5.5: An illustration of the plasma sheath formed at the intersection between the electride and the graphite in an electride cathode with a $1.42 \mathrm{~mm}$ diameter orifice. Due to the alignment of the Fermi levels, the plasma sheath above the electride is $3.86 \mathrm{~V}$ larger than the one over the graphite.

For the plasma over the graphite, an $\boldsymbol{f}_{\boldsymbol{n}}$ of 0.05 was assumed in equation (5.20). Given the calculated sheath potential of $11.6 \mathrm{~V}$ for an electride cathode with a $1.42 \mathrm{~mm}$ diameter orifice, the centerline plasma potential is found from equation (5.22) to be $18.4 \mathrm{~V}$. Using the centerline potential and the increased sheath potential above the electride, the $\boldsymbol{f}_{\boldsymbol{n}}$ for the plasma directly above the electride is calculated to be 0.27 . If the assumption is 
made that the sheath remains unchanged over the entire area of the electride sliver, this value of $\boldsymbol{f}_{\boldsymbol{n}}$ can now be used to estimate the density of the plasma above the electride, which allows for the calculation of the power deposited into the electride surface by the plasma. Using the thermal model for the electride sliver described previously and the sheath potential illustrated in Figure 5.5, the electride temperature is calculated to be $773{ }^{\circ} \mathrm{C}$. This result indicates that the electride surface is $35{ }^{\circ} \mathrm{C}$ cooler than the measured barrel temperature. As such, the power flows indicated in Figure 5.3 are reversed. Figure 5.6 shows the same energy balance with corrected directions of power flow. Because the work function of the electride is low and the surface area in contact with the plasma is small, the heating processes from the plasma are dominated by the cooling effect of the electron emission. The results of the model indicate that for an electride hollow cathode, the barrel temperature is not equal to the emission surface temperature. 


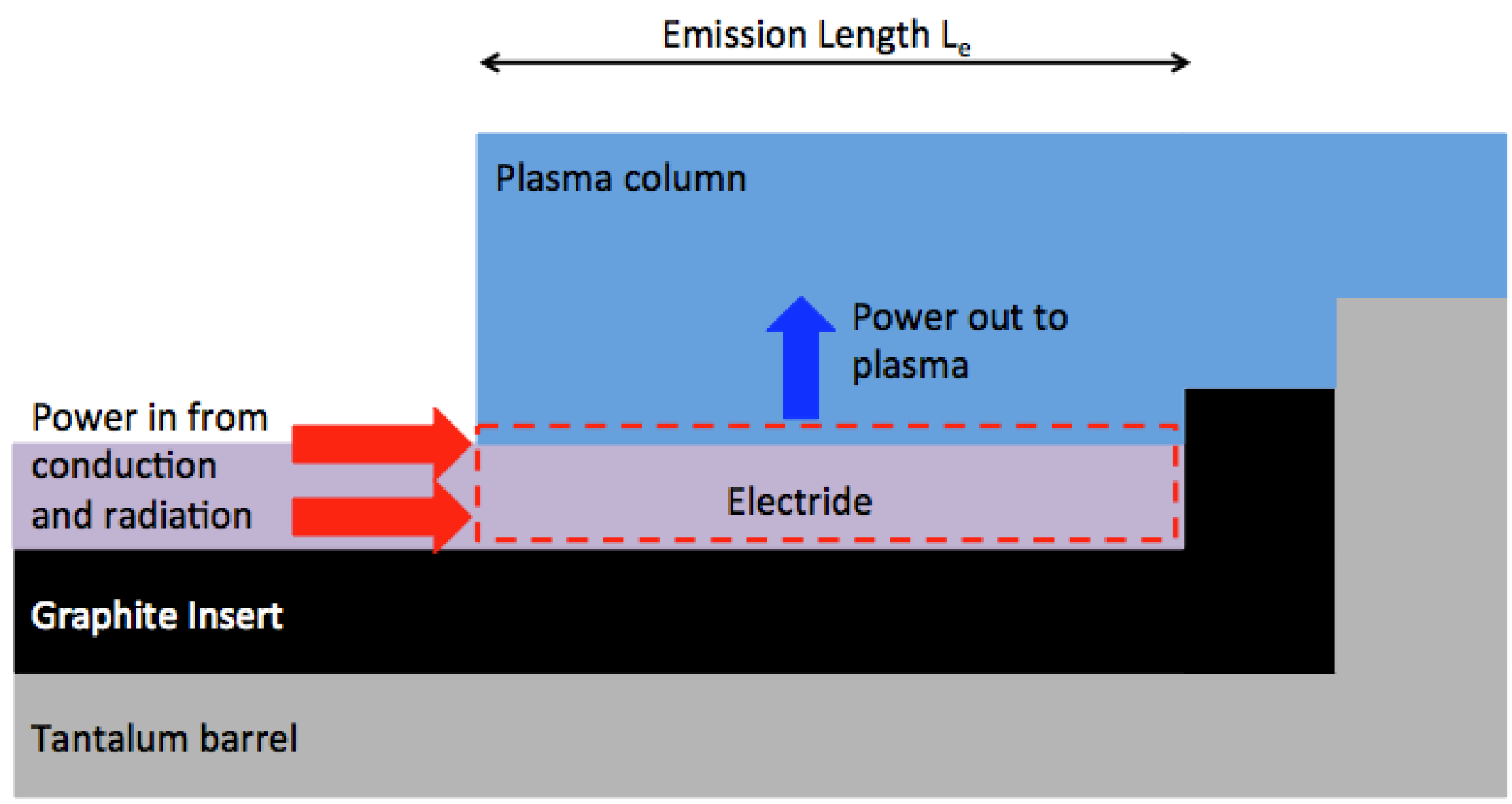

Figure 5.6: Schematic of the energy balance applied to the control volume drawn at the electride surface with corrected directions of heat flow. 


\subsection{Electride Hollow Cathode Model Results}

In the course of the model description presented in Section 5.2, the results for an electride hollow cathode with a $1.42 \mathrm{~mm}$ diameter orifice were presented. The model was also exercised for a $0.76 \mathrm{~mm}$ orifice and a $2.03 \mathrm{~mm}$ orifice. For the two cathodes with the larger orifices, orifice heating was neglected and only orifice plate heating was considered. The validity of this assumption was discussed in Section 5.2. The experimental results indicate that it is possible that orifice heating should not be so easily discounted for the cathode with the smallest orifice. Alternatively, the increase in operating temperature could be due to the higher pressure inside the cathode, which in turn would lead to a shorter emission length, focusing the density of the plasma much closer to the orifice, and as a result, closer to where the thermocouple was placed [5].

To grasp how important orifice heating may be in the cathode with the $0.76 \mathrm{~mm}$ diameter orifice, an approximation of its magnitude can be made. In the NSTAR cathode when operating at the TH15 throttle point $(13.1 \mathrm{~A}, 3.7 \mathrm{sccm}, 0.76 \mathrm{~mm}$ orifice diameter and $1.02 \mathrm{~mm}$ orifice length), the resistance of the orifice plasma is predicted (and experimentally-verified) to be $0.31 \Omega$, which corresponds to a power loss of $53 \mathrm{~W}[6]$. Detailed two-dimensional modeling shows that half of this power loss goes to heating the cathode through the orifice, while the other half is convected out of the cathode through the plume [6]. If the assumption is made that the electride cathode orifice is $0.5 \Omega$, which is increased slightly due to the lower plasma density (because of the decreased discharge current) and longer orifice length, the power loss through the orifice would be $5.45 \mathrm{~W}$. If the same $50 \%$ heating assumption is made, this corresponds to an orifice heating value of $2.72 \mathrm{~W}$. This number is not drastically different than the values calculated in the other two 
cathodes for orifice plate heating, which indicates that the measured elevated operating temperature for the smallest diameter cathode is more likely due to a highly non-uniform plasma density distribution than a different heating mechanism. For the model results presented below, $2.72 \mathrm{~W}$ is used in place of orifice plate heating for the results for the 0.76 mm orifice cathode.

Table 5.2 and Table 5.3 show the geometry of the two other cathodes modeled. They are identical to the $1.42 \mathrm{~mm}$ orifice cathode, with the exception of the orifice diameters. The operating temperature measured for the cathode with the $0.76 \mathrm{~mm}$ diameter orifice was $909^{\circ} \mathrm{C}$ and for the cathode with the $2.03 \mathrm{~mm}$ diameter orifice was $781^{\circ} \mathrm{C}$.

Table 5.2: Geometry used to model electride hollow cathode with $0.76 \mathrm{~mm}$ diameter orifice.

\begin{tabular}{|l|c|c|}
\hline Inner radius of tantalum barrel & $\boldsymbol{r}_{\boldsymbol{i}, \text { barrel }}$ & $2.67 \mathrm{~mm}$ \\
\hline Outer radius or tantalum barrel & $\boldsymbol{r}_{\boldsymbol{o}, \text { barrel }}$ & $3.18 \mathrm{~mm}$ \\
\hline Inner radius of graphite insert & $\boldsymbol{r}_{\boldsymbol{i}, \text { graphite }}$ & $1.27 \mathrm{~mm}$ \\
\hline Outer radius of graphite insert & $\boldsymbol{r}_{\boldsymbol{o}, \text { graphite }}$ & $2.54 \mathrm{~mm}$ \\
\hline Orifice diameter & $\boldsymbol{d}_{\text {orifice }}$ & $0.76 \mathrm{~mm}$ \\
\hline Orifice length & $\boldsymbol{I}_{\text {orifice }}$ & $1.37 \mathrm{~mm}$ \\
\hline
\end{tabular}

Table 5.3: Geometry used to model electride hollow cathode with $2.03 \mathrm{~mm}$ diameter orifice.

\begin{tabular}{|l|c|c|}
\hline Inner radius of tantalum barrel & $\boldsymbol{r}_{\boldsymbol{i} \text {, } \text {, arrel }}$ & $2.67 \mathrm{~mm}$ \\
\hline Outer radius or tantalum barrel & $\boldsymbol{r}_{\boldsymbol{o} \text {, barrel }}$ & $3.18 \mathrm{~mm}$ \\
\hline Inner radius of graphite insert & $\boldsymbol{r}_{\boldsymbol{i} \text {, } \text { graphite }}$ & $1.27 \mathrm{~mm}$ \\
\hline Outer radius of graphite insert & $\boldsymbol{r}_{\boldsymbol{o} \text {, graphite }}$ & $2.54 \mathrm{~mm}$ \\
\hline Orifice diameter & $\boldsymbol{d}_{\text {orifice }}$ & $2.03 \mathrm{~mm}$ \\
\hline Orifice length & $\boldsymbol{I}_{\text {orifice }}$ & $1.37 \mathrm{~mm}$ \\
\hline
\end{tabular}


Figure 5.7 shows the calculated pressure and neutral density for each of the three orifice sizes.

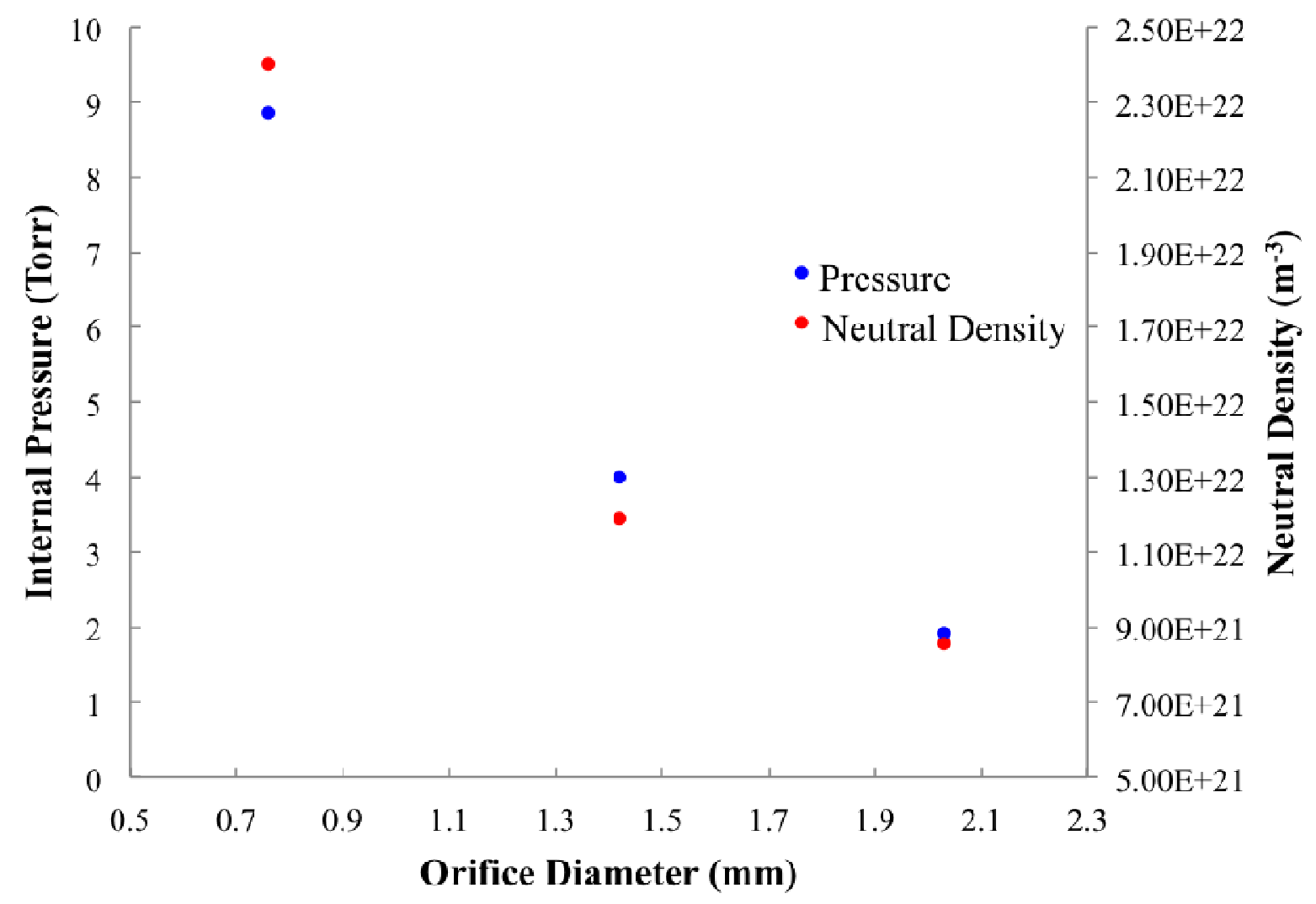

Figure 5.7: The calculated internal pressure and neutral density for the three orifice sizes.

The trends shown in Figure 5.7 are what one would expect. As the orifice size decreases, the interior cathode pressure increases. Because the neutral density is a function of that pressure, it increases as well.

Figure 5.8 shows the calculated electron temperature and sheath potential for each of the three orifice sizes. 


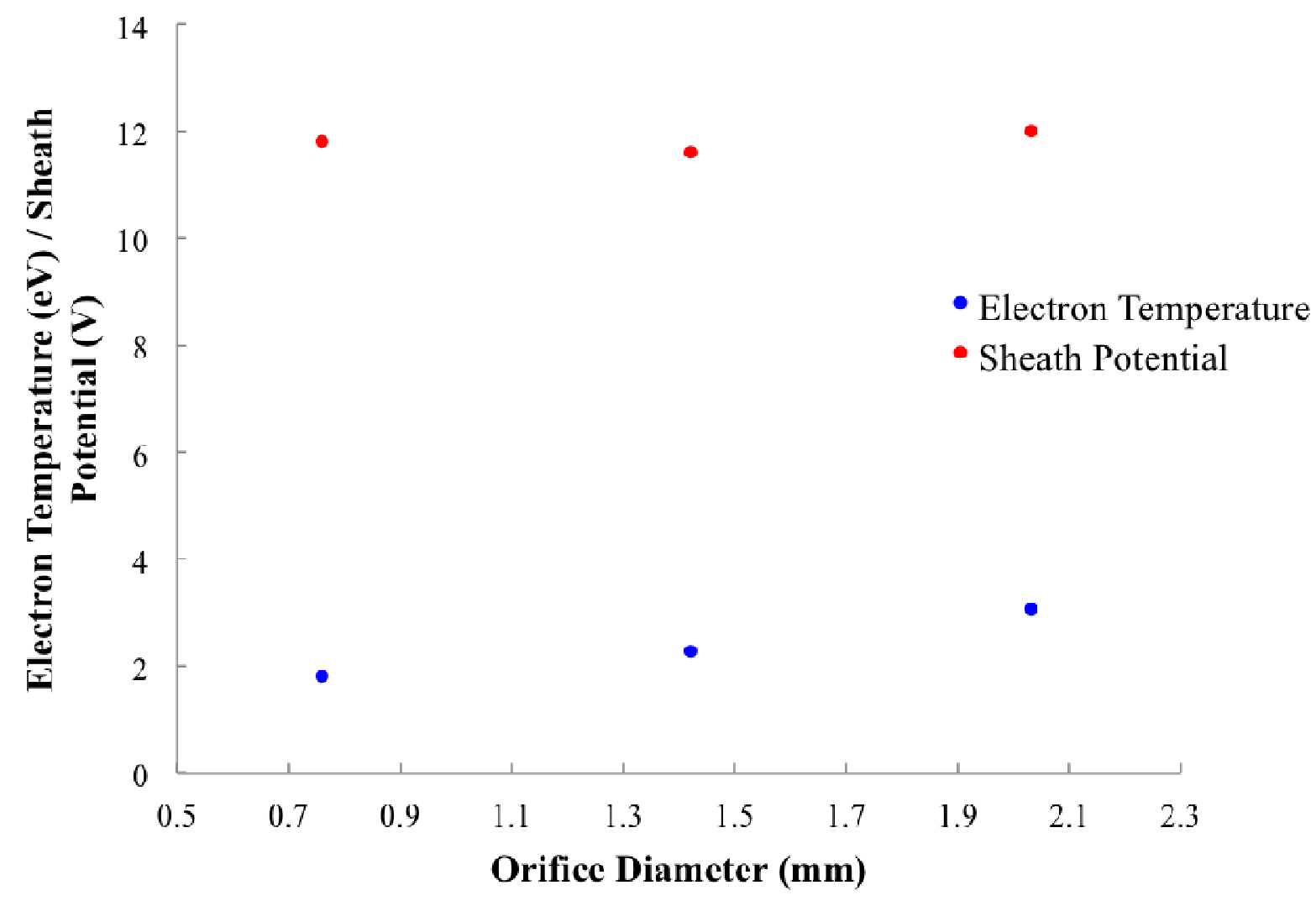

Figure 5.8: The calculated electron temperature and sheath potential (evaluated over the graphite) for the three orifice sizes.

Figure 5.8 shows that the electron temperature increases with orifice diameter. This is expected because the electron temperature is inversely related to the neutral density, which decreases with orifice size. Interestingly, the sheath potential does not change considerably with orifice size. The method to calculate sheath potential uses the heat flow obtained from the thermal model to look at how severe the ion and electron bombardment must be to provide that much power input. Because the operating temperatures of the three cathodes are all within $130{ }^{\circ} \mathrm{C}$ of one another, there is not a dramatic difference between the sheath voltages.

Figure 5.9 shows the calculated plasma density for each of the three orifice sizes. 


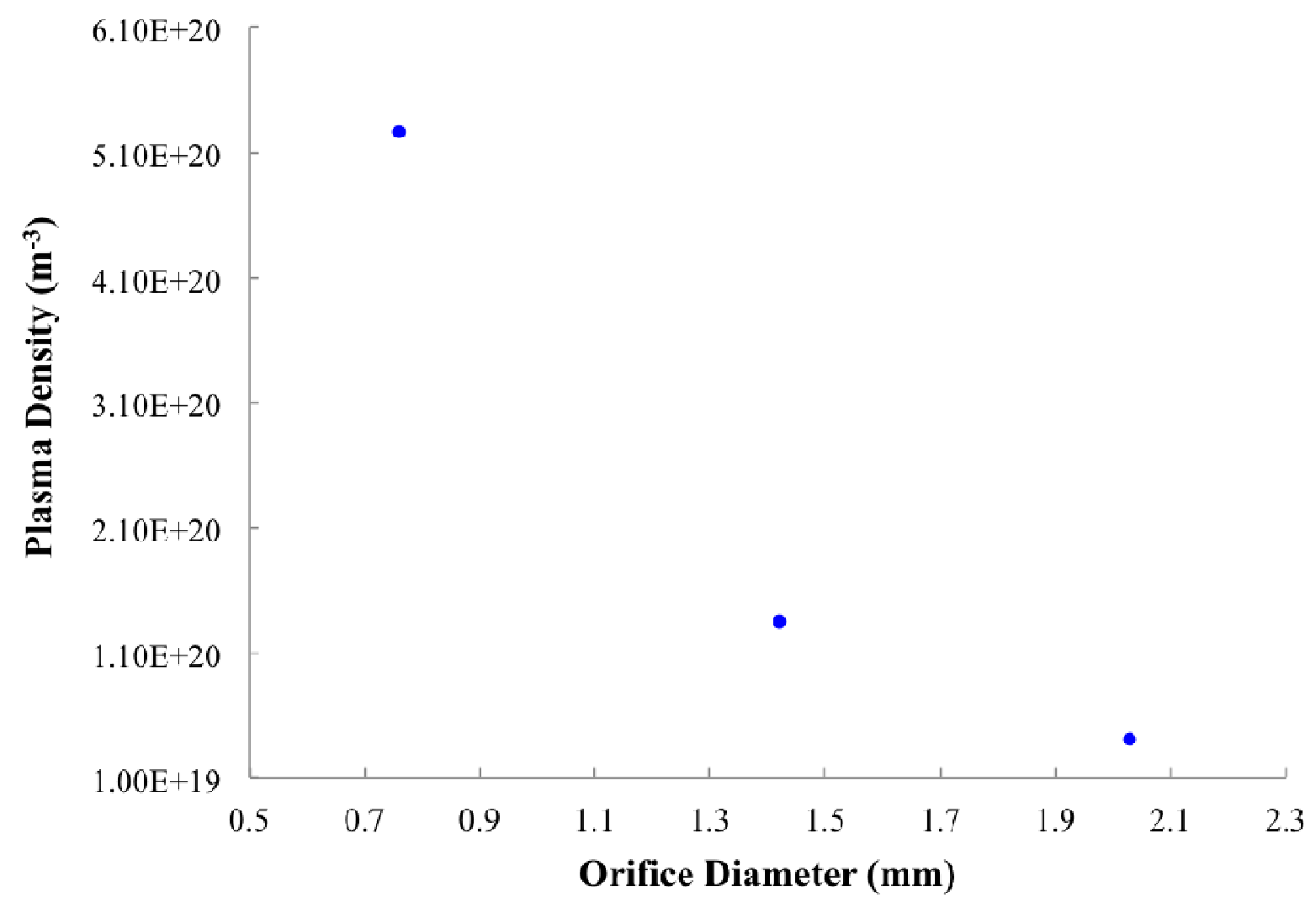

Figure 5.9: The calculated plasma density for the three orifice sizes.

As expected, the plasma density increases with decreasing orifice size.

Figure 5.10 shows the calculated electride surface temperature and the difference between the calculated surface temperature and the measured wall temperature for each of the three orifice sizes. 


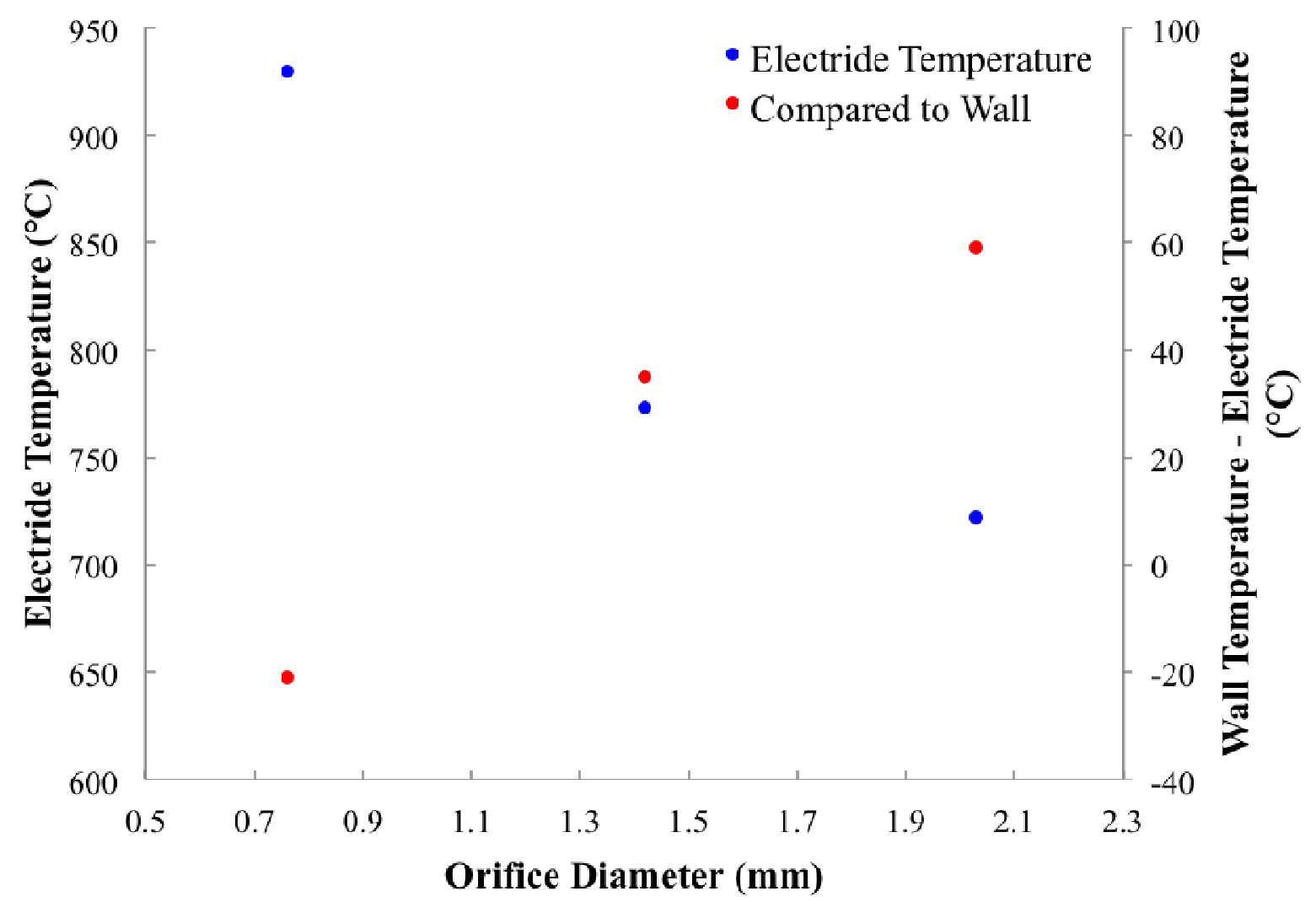

Figure 5.10: The calculated electride surface temperature and the difference between the surface temperature and the measured barrel temperature for the three orifice sizes.

The electride temperatures of all three cathodes are within $60{ }^{\circ} \mathrm{C}$ of the measured wall temperature. Interestingly, the electride surface in the cathode with the smallest orifice is predicted to be hotter than its surroundings by approximately $20^{\circ} \mathrm{C}$, whereas the electride surfaces in the two larger cathodes are predicted to be cooler than their surroundings. It is difficult to draw hard conclusions from this result, due to possible inaccuracies from simplifications in the thermal model and plasma non-uniformity that are not reflected in the zero-dimensional model. 


\section{References}

[1] C. M. Ferreira and J. L. Delcroix, J. Appl. Phys. 49, 2380 (1978).

[2] D. E. Siegfried, A Phenomenological Model for Orificed Hollow Cathodes, Colorado State University, 1983.

[3] D. E. Siegfried and P. J. Wilbur, AIAA J. 21, 5 (1983).

[4] I. Katz, J. Polk, I. Mikellides, D. Goebel, and S. Hornbeck, in 29th Int. Electr. Propuls. Conf. (Princeton, New Jersey, 2005).

[5] I. Katz, I. Mikellides, D. Goebel, and J. Polk, in 30th Int. Electr. Propuls. Conf. (Florence, Italy, 2007), pp. 1-9.

[6] D. Goebel and I. Katz, Fundamentals of Electric Propulsion: Ion and Hall Thrusters (John Wiley \& Sons Inc, 2008).

[7] J. S. Miller, S. H. Pullins, D. J. Levandier, Y. Chiu, and R. a. Dressler, J. Appl. Phys. 91, 984 (2002).

[8] D. Rapp and P. Englander-Golden, J. Chem. Phys. 43, 1464 (1965).

[9] A. Salhi, R. Meyers, and J. Turchi, 23rd Int. Electr. Propuls. ... Paper No. 93 (1993).

[10] K. Krishnan and S. Jain, Nature 169, (1952).

[11] D. A. Neamen, Semiconductor Physics and Devices, Fourth (McGraw-Hill, New York, NY, 2012). 


\section{Conclusions and Future Work}

\subsection{Summary of Fabrication Process}

A one-step fabrication process was developed to produce C12A7 electride. The precursors were mixed and placed in a graphite crucible in a furnace. The furnace was heated in vacuum to $1700{ }^{\circ} \mathrm{C}$, and allowed to cool slowly. The resulting product was metallic in appearance and conductive. An analysis of the atomic composition of the material surface by x-ray photoelectron spectroscopy resulted in the expected aluminum to calcium ratio. Additionally, examination of the fine structure of the XPS results narrowed the material components down to calcium oxide and either alumina or aluminum hydroxide. Electron paramagnetic resonance of the product identified unpaired electrons in the compound, and the EPR peak shape was indicative of some delocalization of the

electrons, signifying a semi-metallic mode of conduction. X-ray diffraction taken of the sample showed no peaks corresponding to a compound other than graphite, which pointed to the production of the amorphous form of C12A7 electride rather than the fully crystalline.

The work function of the produced electride was measured at low temperature using a diode apparatus, in which the sample was heated to a known temperature and the current emitted to a biased anode plate was quantified. The lowest work function measured was $0.76 \mathrm{eV}$, which corresponded to an emitted current density of $0.15 \mathrm{~A} / \mathrm{m}^{2}$. The emitted current was observed to decrease over time to below a measurable value, and did not recover after a return to room temperature and reheating. Additional current was only observed at higher temperatures, although, after a period of tens of minutes, it also 
decreased to zero. It is thought that defect electronic states near the surface were being depleted, giving rise to an increased work function. An unidentified process within the hollow cathode during operation appears to prevent emission degradation with time.

An electride hollow cathode insert was fabricated using an electride sliver cut from the crucible in which the electride was formed. It was found that a continuous emission surface resulted in much more stable and consistent cathode operation. No special precautions were taken to keep the electride surface pristine during its preparation, and there appeared to be no detriment from the lack of such provisions, however, a graphite liner placed inside the tantalum tube was discovered to be necessary. Graphite is known to supply an anionic template that keeps the nanocages stable when electride is heated and cooled, and it is likely a similar process occurred inside the cathode when electride was in contact with the graphite. The operation produced by a fresh, never-used electride insert was characterized by increased anode voltages and cathode operating temperatures, both of which decreased to reproducible values after between three and four multi-hour operations that were separated by cool down periods.

\subsection{Summary of Experimental Results}

An electride hollow cathode was constructed from a tantalum barrel that employed a graphite liner where the electride was placed. It required only $400 \mathrm{~V}$ to start with 50 sccm of xenon flowing. The start-up mechanism of the final prototype was likely due to a cascade ionization effect triggered by electrons emitted from the electride surface at room temperature. A single insert was operated in a cathode for over 60 hours, spread across a 2-month period. This duration involved 20 restarts from room temperature, as well as 11 exposures to atmosphere. 
Cathode bodies with three different orifice sizes were operated and compared using the same insert. It was found that the cathode with the smallest orifice size ran at a temperature approximately $100{ }^{\circ} \mathrm{C}$ hotter than the cathodes with the two larger orifices at a discharge current of 2.8 A. The smaller orifice increased the pressure in the cathode and moved the peak plasma density to a location near the thermocouple, which would increase the power deposited in that area by plasma bombardment of the surface. Similar temperatures were observed for all three cathodes at lower discharge currents. The low internal plasma densities that correspond to low discharge currents may have negated any significant heating effect from spatially different plasma densities.

At Busek Co. Inc., an electride cathode was operated using iodine vapor as the propellant. No degradation of the electride surface due to interaction with the iodine that inhibited emission was observed. The cathode started at room temperature on iodine, and ran for over 20 hours. The testing with iodine included eight restarts from room temperature as well as one exposure to atmosphere.

\subsection{Summary of Modeling Effort}

A zero-dimensional phenomenological model was constructed to gain insight into the plasma conditions inside an electride hollow cathode based on the measured operating temperature. An energy balance involving a heat transfer model was applied to a control volume on the outer surface of the tantalum barrel and graphite liner. An additional energy balance applied to the internal plasma was used in conjunction with the computed power deposition at the graphite liner surface to calculate the electron temperature, sheath potential, and average plasma density. Using these properties along with the experimentally measured work function of $0.76 \mathrm{eV}$, the power deposited into the electride 
surface could be approximated, which, combined with a heat transfer model, allowed for the prediction of the electride surface temperature.

The model, combined with experimentally measured operating temperatures, was used to calculate the plasma properties for cathodes with three different orifice sizes. Decreasing orifice size, as expected, increased the internal cathode pressure and decreased electron temperature. The orifice size was not found to have a significant effect on the sheath potential. For the smallest orifice, the electride surface temperature was predicted to be $20^{\circ} \mathrm{C}$ hotter than its surroundings, whereas the surface temperatures of the electride inserts in the two larger orificed cathodes were predicted to be $\sim 40{ }^{\circ} \mathrm{C}$ cooler than their surroundings.

\subsection{Future Work}

Further characterization of the electride surface could provide insight into the structure of the material and its emission properties. A comparison of XRD, XPS, and EPR spectra taken before and after the electride surface has operated within a hollow cathode would give information about any changes undergone by the surface due to the cathode operation. A work function test apparatus that exposed the surface to a plasma similar to that found within the cathode could look deeper into the apparent recovery of the low work function emission after cathode operation.

As described in Chapter 3, the electride material only covered a small fraction of the cathode interior. Consequently, an annular electride insert in which the internal walls were completely coated in electride would be highly desirable. There would be a greater emission surface area, which could lower the operating temperature of the cathode. Additionally, the plasma would not bombard high work function surfaces such as graphite, 
which could also lead to a lower operating temperature. There are several ways that such an insert could be fabricated. A hole could be machined through the center axis of a solid electride cylinder, at which point it could be inserted into a cathode barrel or graphite liner. Alternatively, an insert could be constructed using the sliver method described herein and put in a cathode. The cathode could then be operated at discharge currents that increased its internal temperature to above the melting point of the electride. Because of its amorphous nature, it is expected that the electride would continue to emit. While running, the cathode could be slowly rotated about its axis, resulting in the coating of the graphite liner by the electride. Other similar methods could be used where liquid electride was supplied to an operating cathode.

Higher current electride cathodes, on the order of $100 \mathrm{~A}$, could have a great deal of use in future high power electric propulsion systems. These cathodes could be made in several ways. The fabrication process described herein could simply be scaled up, to produce a $1.27 \mathrm{~cm}-2.54 \mathrm{~cm}$ cathode similar to LaB6 cathode development efforts. Alternatively, multiple inserts identical to those described herein could be placed in a large barrel.

Additional experimental tests are needed before an electride hollow cathode is perceived to be as reliable as the current state-of-the-art by the electric propulsion community. The first step in demonstrating electride cathode durability may be a longer duration test, on the order of several hundred hours. Further testing of an electride cathode in conjunction with a thruster is also vital. Lastly, given the observed durability of the electride surface, it would be worthwhile to operate an electride cathode in non-ideal environments, especially with an increased oxygen background pressure. If the electride 
can continue to emit in the presence of oxygen, then expensive, ultra-high purity propellants would no longer be necessary.

The zero-dimensional model could be greatly improved by using data from fastmoving probe measurements of the electride surface temperature and the axial plasma density. Operating temperatures acquired from an array of thermocouples placed axially along the barrel surface would also be valuable. These experimental results could lead to the development of one and two-dimensional models that better represented the spatial plasma variations. The additional thermocouples would also improve the heat transfer calculations, leading to more accurate calculations of plasma properties. 


\section{APPENDIX A}

An initial prototype electride hollow cathode was taken to the Air Force Research Laboratory at Edwards Air Force base to operate in conjunction with a $6 \mathrm{~kW}$ Hall thruster [1]. It was mounted parallel with the thruster approximately $5 \mathrm{~cm}$ away from the outer edge of the thruster housing with the orifice plate at the thruster exit plane, as shown in Figure A. 1. The thruster anode voltage and gas flow were turned on, and the gas flow to the cathode increased until it started. It took several arcs for the cathode to maintain a discharge. The thruster started simultaneous with the cathode.

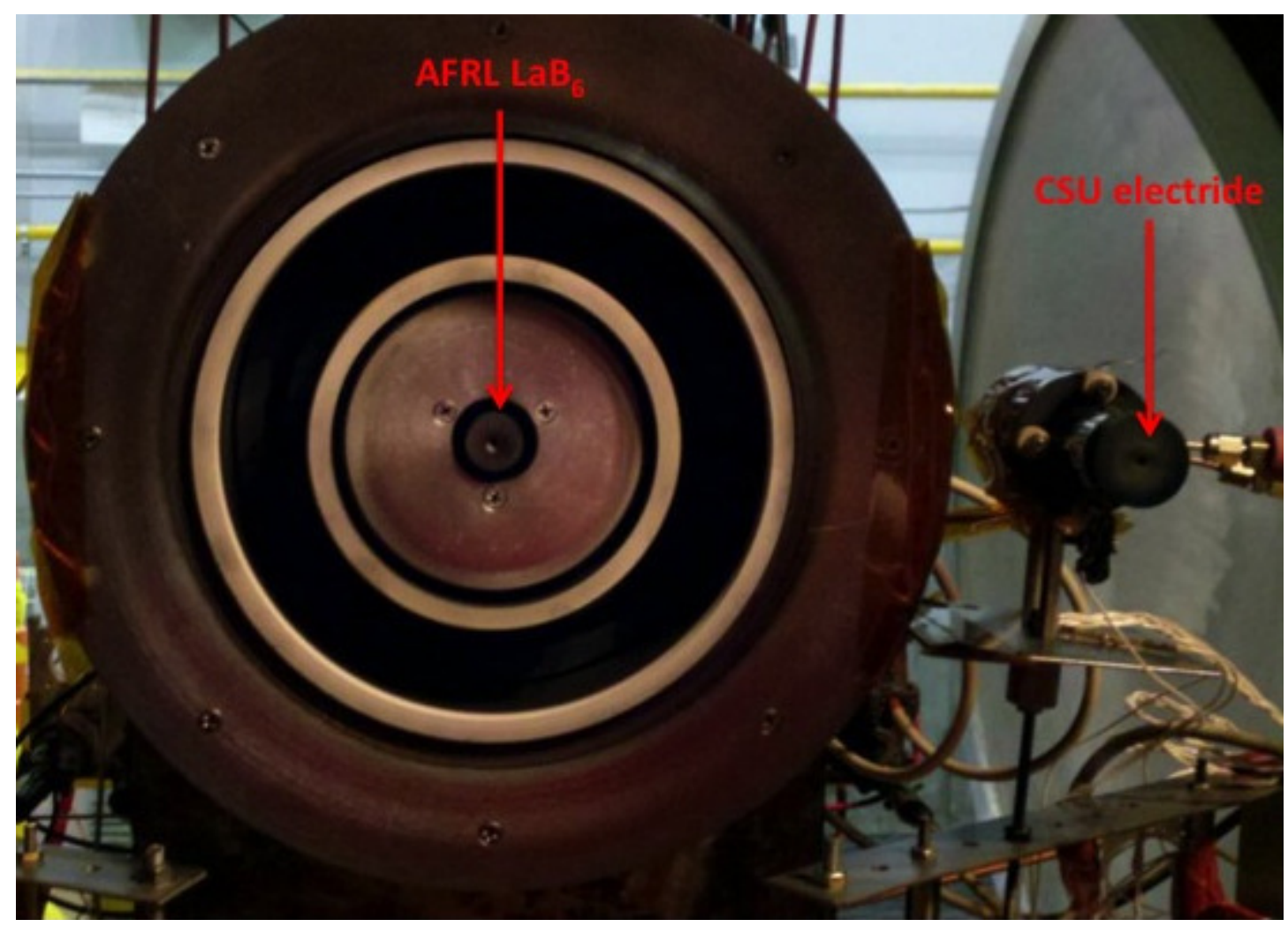

Figure A. 1: H6 thruster with mounted electride hollow cathode.

The cathode operation with the thruster was similar to that observed in the diodeconfiguration. Specifically, the operation consisted of multiple and visible ejection of material, as shown in Figure A. 2. 


\section{ด8-02-2012 18:25:28}

\section{个 \\ Electride \\ hollow cathode}

Figure A. 2: AFRL H6 Hall thruster operating in conjunction with electride hollow cathode. Mass flow rate to cathode is $10 \mathrm{sccm}$ of xenon, thruster operating conditions are $9 \mathrm{~A}$ and $2.71 \mathrm{~kW}$. Cathode is operating in a relatively stable mode.

The cathode operated for approximately 30 minutes before winking out for the first time. It was easily relit, after which point it operated for an additional several minutes before winking out again. The wink-outs increased in frequency, until the cathode could no longer be started. It is hypothesized that the material ejected during the unstable modes of operation built up to form an electrical contact between the keeper and the cathode barrel. This prevented the application of a high voltage to the keeper, which made it impossible to start the cathode.

The H6 thruster was mounted on a reverse-pendulum thrust stand configuration. The total efficiency of the thruster can be calculated according to

$$
\eta_{T}=\frac{T^{2}}{2 \dot{m} P_{i n}}
$$


where $\eta_{T}$ is the total efficiency, $T$ is the thrust, $\dot{m}$ is the total mass flow rate, and $P_{\text {in }}$ is the total electrical power into the thruster [2]. Two terms are likely to change when switching between a center-mounted traditional LaB6 cathode and an outer-mounted electride cathode. If the cathode required a higher voltage to obtain an adequate discharge current, that would be reflected in an increased $P_{i n}$. Even if both cathodes operated identically, $P_{\text {in }}$ was expected to increase with the electride cathode due to the difference in mounting positions. The externally-mounted electride cathode was farther away from the channel, which required a higher voltage for the electrons to reach the anode. The other term that could vary between the cathodes was $\dot{m}$, which was based on the propellant utilization inside the cathodes. If the electride cathode required a greater amount of propellant than the LaB6 cathode to emit the discharge current, then that would be reflected in an increase of $\dot{m}$.

It was found that the thruster, when operating in conjunction with the electride cathode, had a total efficiency of $52.6 \%$ at an anode voltage of $300 \mathrm{~V}$ and an operating power of $2.7 \mathrm{~kW}$. In comparison, when the thruster operated with the center-mounted LaB6 cathode under the same conditions, it exhibited a total efficiency of $54.4 \%$. An approximate $2 \%$ discrepancy was expected between the two numbers, given the difference in mounting positions. As such, it was concluded that there was no meaningful difference in total efficiency between a $6 \mathrm{~kW}$ Hall thruster operating with a traditional LaB6 cathode and the same thruster operating with an electride cathode. 


\section{References}

[1] D. L. Brown, Investigation of Low Discharge Voltage Hall Thruster Characteristics and Evaluation of Loss Mechanisms, University of Michigan, 2009.

[2] D. Goebel and I. Katz, Fundamentals of Electric Propulsion: Ion and Hall Thrusters (John Wiley \& Sons Inc, 2008). 